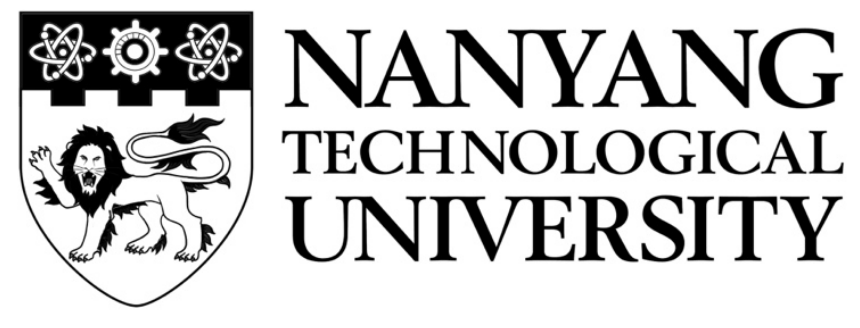

\title{
EFFICIENT AND SECURE MOBILITY SUPPORT IN 6LOWPAN NETWORKS
}

\author{
QIU YUE \\ SCHOOL OF ELECTRICAL AND ELECTRONIC ENGINEERING \\ 2017
}





\title{
EFFICIENT AND SECURE MOBILITY SUPPORT IN 6LOWPAN NETWORKS
}

\author{
QIU YUE
}

SCHOOL OF ELECTRICAL AND ELECTRONIC ENGINEERING

\author{
A thesis submitted to the Nanyang Technological University \\ in partial fulfillment of the requirement for the degree of \\ Doctor of Philosophy
}





\section{Acknowledgements}

First and foremost, I would like to express my sincere gratitude to my supervisor, Professor Ma Maode, for his guidance, expertise, patience and understanding throughout my Ph.D. period. I appreciate his profound knowledge and academic support for overcoming the obstacles in my study, and his assistance in developing my writing skills. The kind encouragement from him imbues me with self-confidence and has stimulated my enthusiasm in the research field. It is a great honor to be a Ph.D. student under his supervision. I also wish to express my appreciation to Prof. Lu Rongxing for his constructive advice on my research topic.

I am indebted to my group member (Mr. Chen Shuo, Dr. Zhang Zhenjing, Mr. Alican Ozhelvaci and Mr. Zhang Lvye) for the discussions and helpful suggestions on my research. I am also very grateful to my dear friends (Ms. Yap Lee Jong, Ms. Ni Jun, Dr. Yew Kwang Sing, Ms. Dong Meng, Mr. Huang Cheng and Dr. Hu Hao) for their support and friendships.

Finally, I would also like to gratefully thank my parents, my grandparents and

other family members for their love, encouragement and support which are always a source of inspiration and motivation of my life. 


\section{Table of Contents}

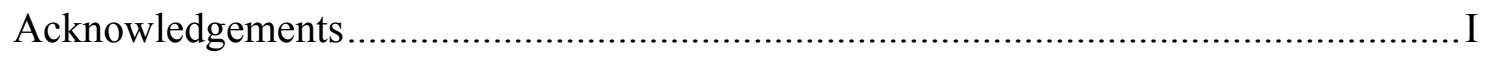

Table of Contents ............................................................................................ III

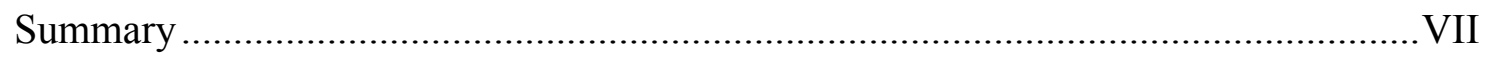

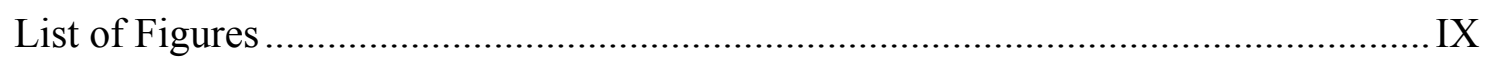

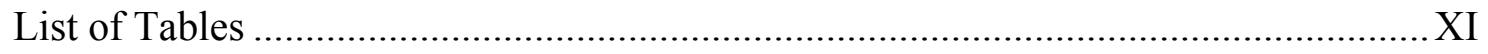

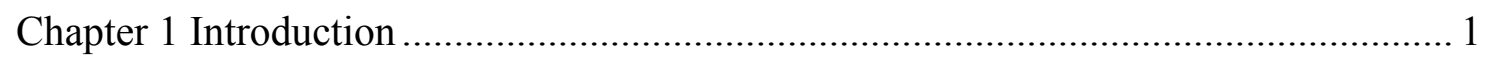

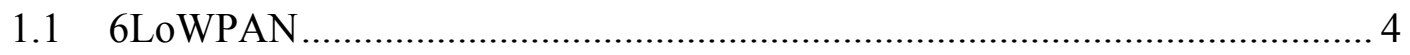

1.1.1 6LoWPAN Architecture ................................................................ 5

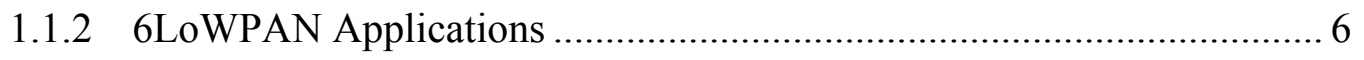

1.1.3 Mobility Protocols in 6LoWPAN .................................................. 7

1.1.4 Security of 6LoWPAN Networks ................................................ 10

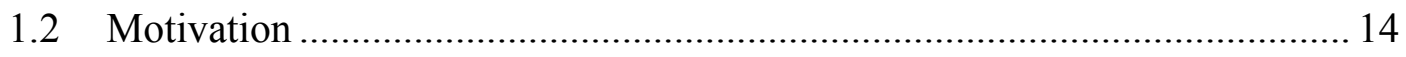

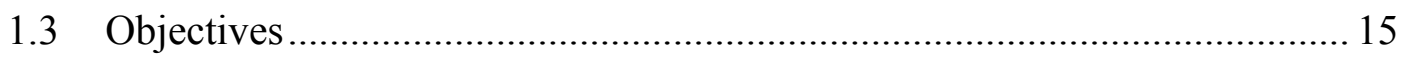

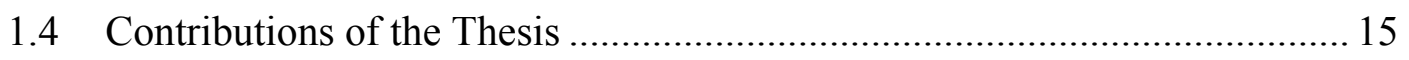

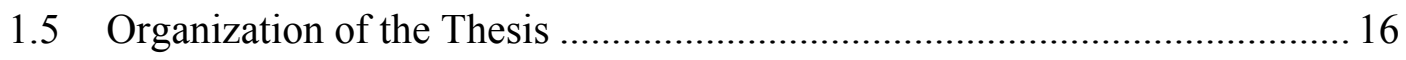

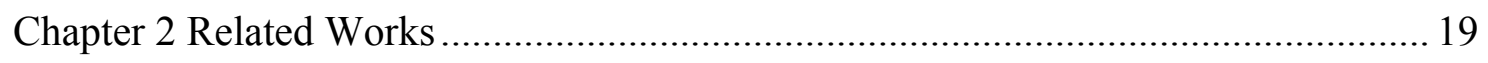

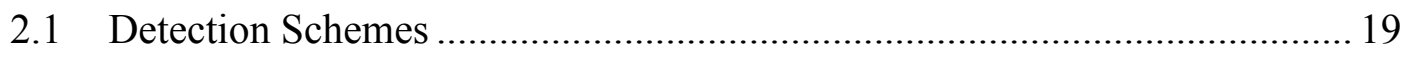

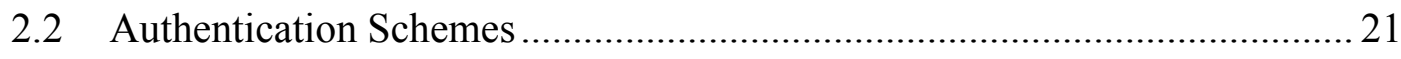

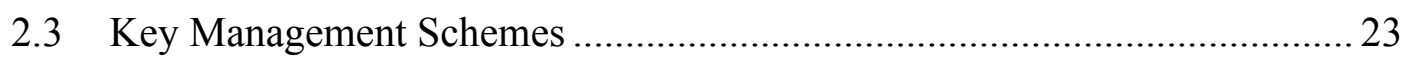

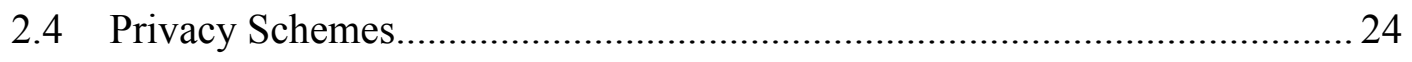

2.5 The Compression of the IPsec Schemes............................................... 25

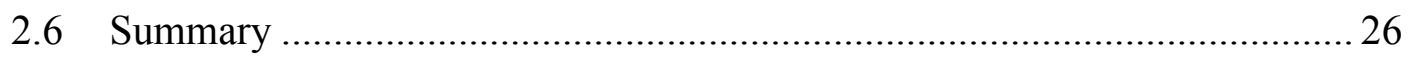


Chapter 3 A Mutual Authentication and Key Establishment Scheme for M2M

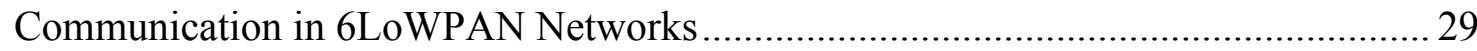

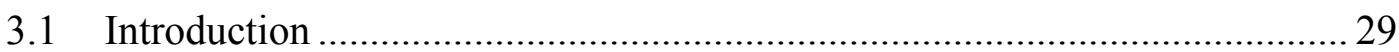

3.2 System Background........................................................................... 30

3.2.1 6LoWPAN System Architecture ................................................................... 30

3.2.2 Elliptic Curve Diffie-Hellman.................................................................... 31

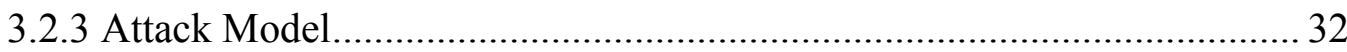

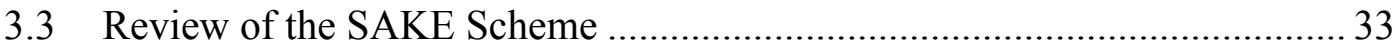

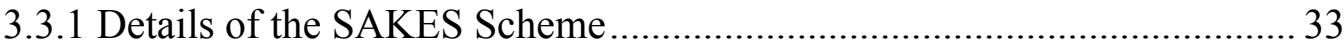

3.3.2 Vulnerabilities of the SAKES Scheme ………………………………...... 35

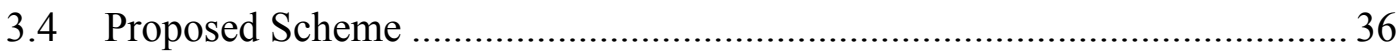

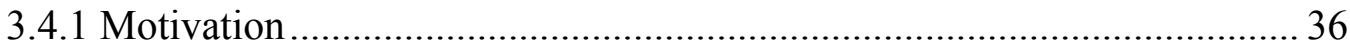

3.4.2 Details of the Proposed EAKES6Lo ............................................................. 36

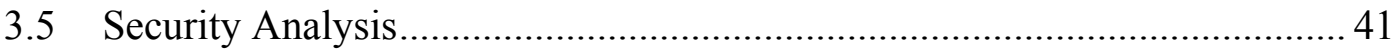

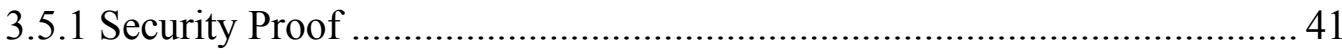

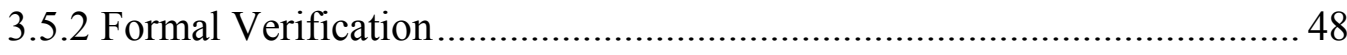

3.5.3 Ability Against Malicious Attacks...........................................................50

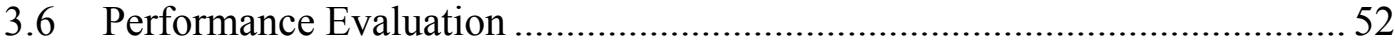

3.6.1 Computational Overhead ....................................................................... 52

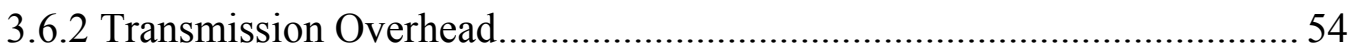

3.6.3 Comparison of Handover Processes …………………………………......57

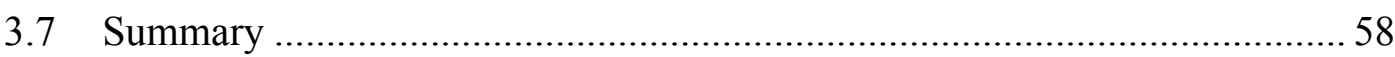

Chapter 4 An Efficient and Secure PMIPv6-based Handover Scheme for 6LoWPAN

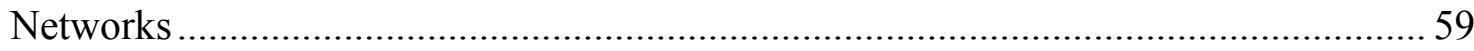

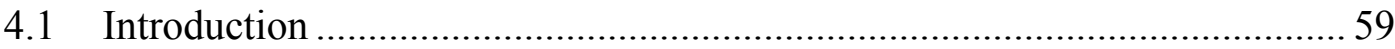




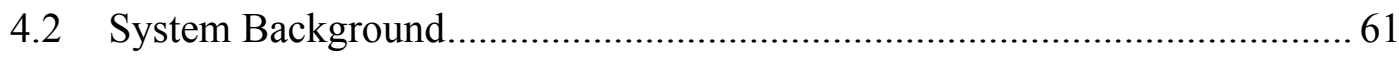

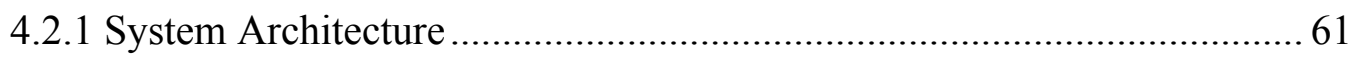

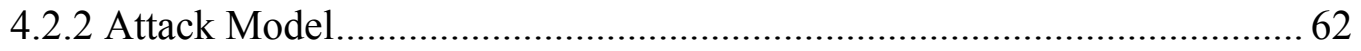

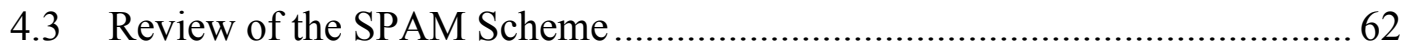

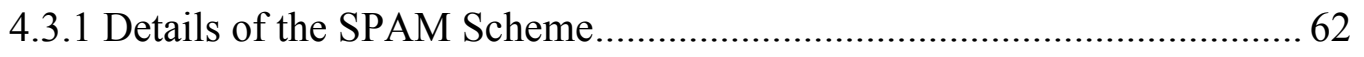

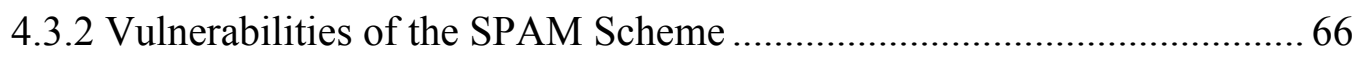

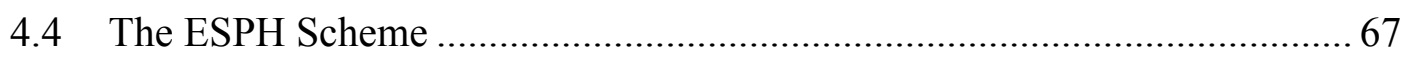

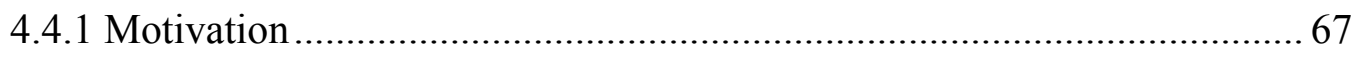

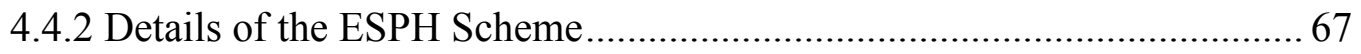

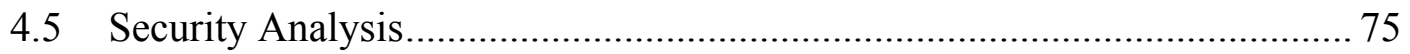

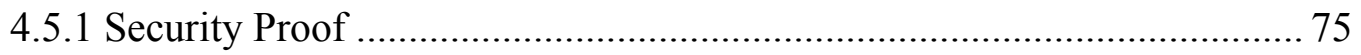

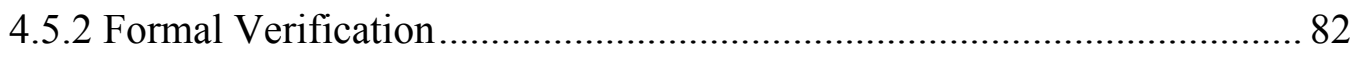

4.5.3 Ability against Malicious Attacks............................................................. 84

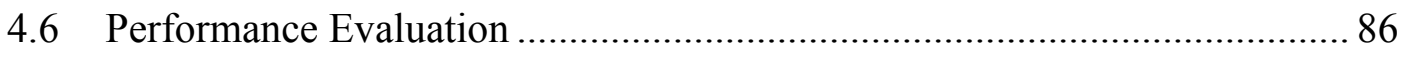

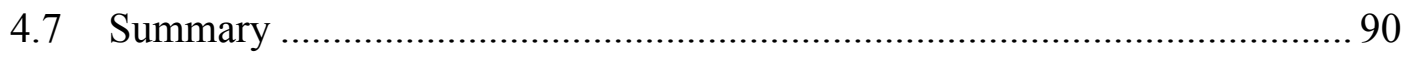

Chapter 5 Secure Group Mobility Support for 6LoWPAN Networks........................... 91

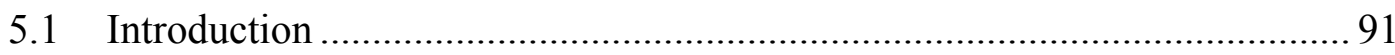

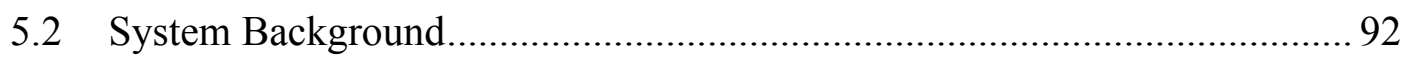

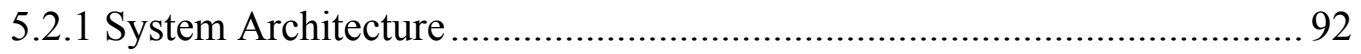

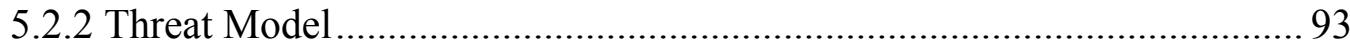

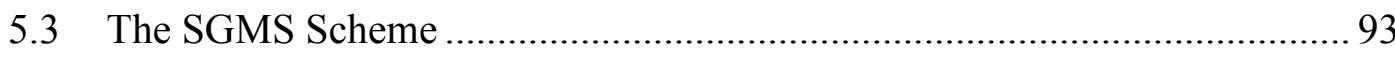

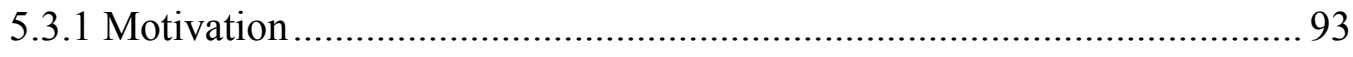

5.3.2 Details of the SGMS Scheme................................................................. 94

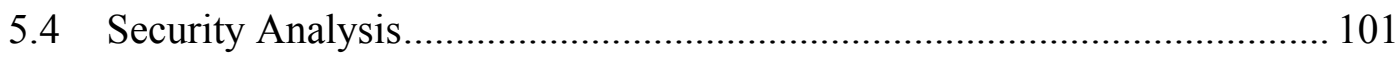




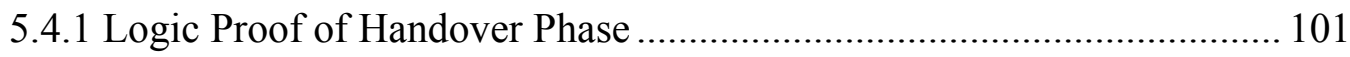

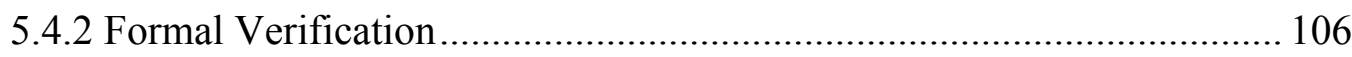

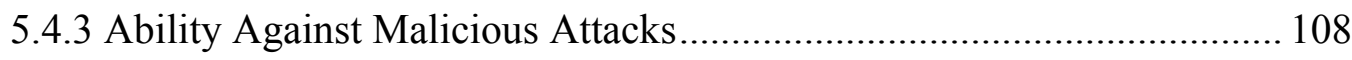

5.5 Performance Evaluation ........................................................................ 110

5.5.1 Computational Overhead ………………………………………….... 110

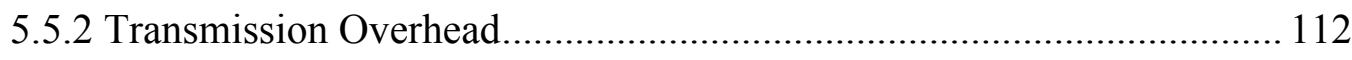

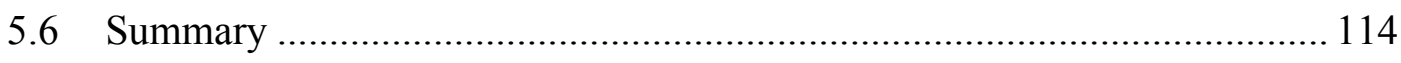

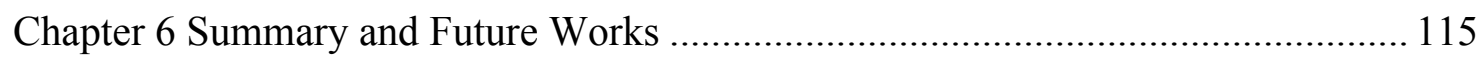

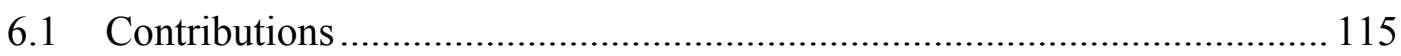

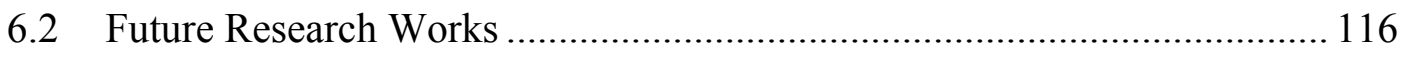

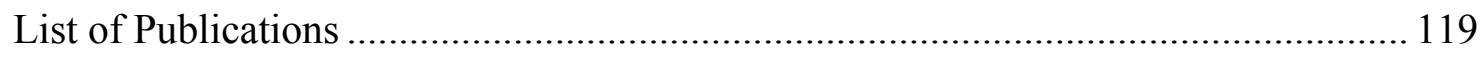

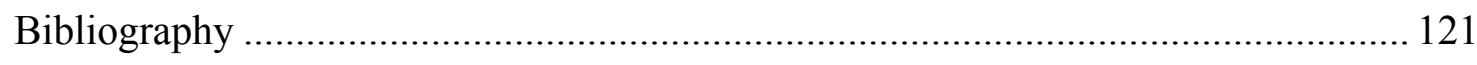

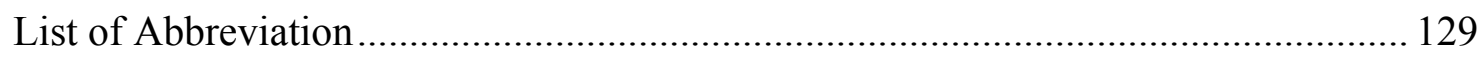




\section{Summary}

The machine-to-machine (M2M) communication, which plays a vital role in the Internet of Things (IoT), allows wireless and wired systems to monitor environments and exchange the information among various machines automatically without human interventions. In future, M2M communication could make many possible applications, such as e-health, smart grids, industrial automation and environmental monitoring, to

produce various wide markets with many opportunities and to bring many more benefits to humans. To promote the development of the IoT and exploit the M2M applications, the Internet Engineering Task Force (IETF) has been developing a standard named Internet Protocol version 6 (IPv6) over low-power wireless personal area networks (LoWPANs) (6LoWPANs) to enable millions of IP-based M2M sensing devices to connect to the open Internet. Since these 6LoWPAN devices are resource constrained in terms of power, memory and battery, traditional security mechanisms which ensure the data security cannot be directly applied. Moreover, due to the reason that the mobile nodes (MNs) would compose a considerable part of future M2M systems, the Proxy Mobile IPv6 (PMIPv6) has been devised by the IETF networkbased localized mobility management (NETLMM) working group to manage the mobility of the MNs with limited resources.

Along with its development, 6LoWPAN networks still face various security threats and trust crises. Due to the large number of interconnected devices in the communication network that are generally deployed in the unattended environment, security is a critical issue for 6LoWPAN networks. Although the 6LoWPAN standard and PMIPv6 protocol have specified many important issues, various security issues have not been addressed. Most of the existing solutions, which focus on the enhancement of the abilities to withstand malicious attacks, are designed for static nodes while the security schemes for protecting the MNs is almost a blank page. 
To fill up this gap, in this thesis, we first introduce the concept and the architecture of the 6LoWPAN in which some mobility support protocols proposed by the IETF can be implemented to realize nodes' mobility. In particular, the security requirements and vulnerabilities in 6LoWPAN are addressed which are important criteria when designing a 6LoWPAN security mechanism. Based on the existing security issues in 6LoWPAN, several security schemes are proposed to enable 6LoWPAN devices to roam in the 6LoWPAN environment efficiently and securely. To meet the requirements of the future IoT that devices can access various network services whenever and wherever needed, supporting a large number of devices is a prerequisite. Therefore, these proposed schemes are designed to be able to provide secure mobility protections for a single node as well as a group of nodes. The logic correctness of the proposed schemes is proved by the protocol logic. The formal verification by the verification tools and the performance evaluation results show that the proposed security schemes for 6LoWPAN networks could not only enhance the security functionalities with the ability to prevent various malicious attacks but also be computationally efficient. 


\section{List of Figures}

Figure 1.1 General Architecture of a CPS ............................................................ 1

Figure 1.2 High-Level Architecture for M2M ...................................................... 3

Figure 1.3 The Reference Model of 6LoWPAN Protocol Stack ................................. 5

Figure 1.4 The 6LoWPAN Architecture................................................................. 6

Figure 3.1 6LoWPAN System Architecture of EAKES6Lo ................................... 31

Figure 3.2 Registration Phase of EAKES6Lo........................................................ 37

Figure 3.3 Authentication and Key Establishment Phase of EAKES6Lo .................... 38

Figure 3.4 Handover Phase of EAKES6Lo ............................................................. 40

Figure 3.5 Output Results of EAKES6Lo using AVISPA ...................................... 49

Figure 3.6 Comparison of Performance Evaluation between EAKES6Lo and SAKES 57

Figure 4.1 6LoWPAN System Architecture of ESPH .......................................... 61

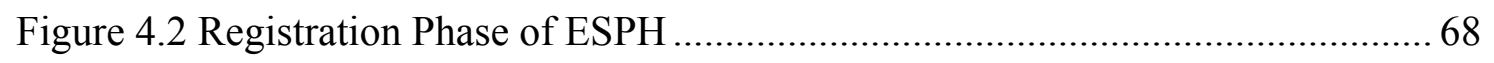

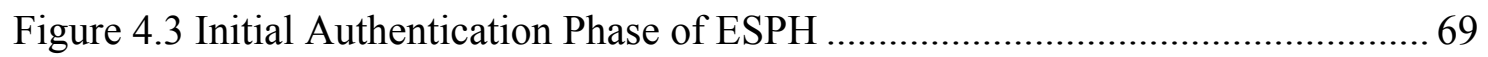

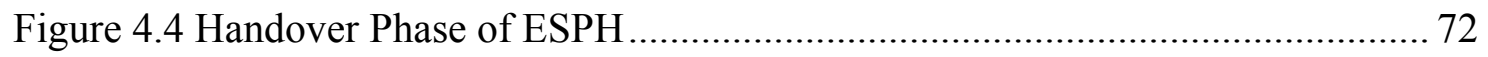

Figure 4.5 An Example of Key Chain Calculation .................................................. 74

Figure 4.6 Output Results of ESPH using AVISPA ............................................. 84

Figure 4.7 Comparison of Performance Evaluation between ESPH and SPAM.......... 90

Figure 5.1 6LoWPAN System Architecture of SGMS ........................................ 92

Figure 5.2 Registration Phase of SGMS ............................................................. 95

Figure 5.3 Initial Authentication Phase of SGMS ............................................... 96

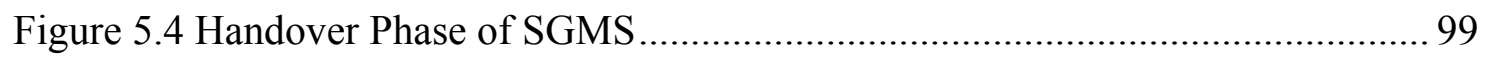

Figure 5.5 Output Results of Fast Authentication in Handover Phase ...................... 107 
Figure 5.6 Comparison of Performance Evaluation between SGMS and SPAM....... 113 


\section{List of Tables}

Table 1.1 Comparison of Mobility Protocols ..................................................... 9

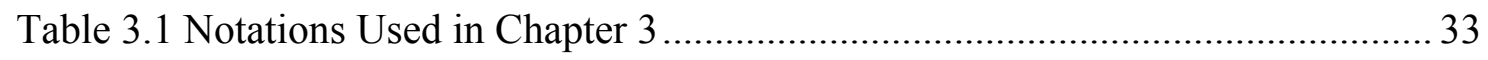

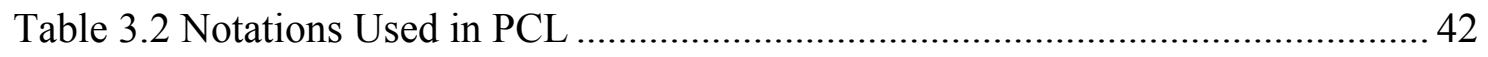

Table 3.3 Comparisons of Security Properties between EAKES6Lo and SAKES ....... 52

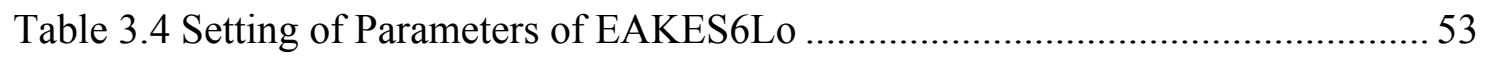

Table 3.5 Comparisons of Ticket-based Schemes .............................................. 58

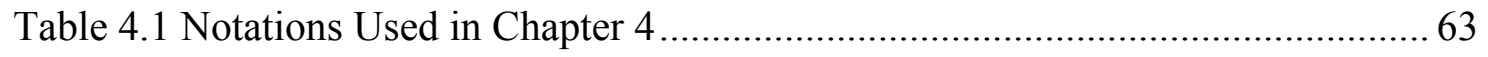

Table 4.2 Comparisons of Security Properties between ESPH and SPAM.................. 86

Table 4.3 Computational Time of Cryptographic Operations ................................... 87

Table 4.4 Comparison of Average Time Consumption between ESPH and SPAM ..... 89

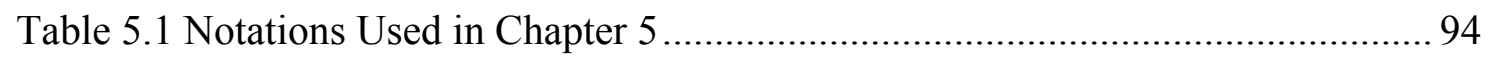

Table 5.2 Notations Used in BAN Logic .......................................................... 102

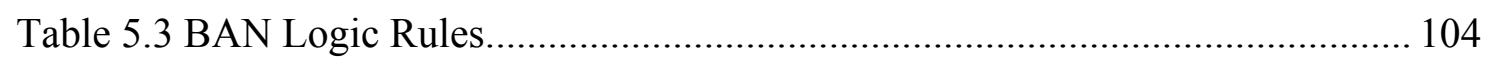

Table 5.4 Comparison of Properties of Authentication Protocols ............................. 109

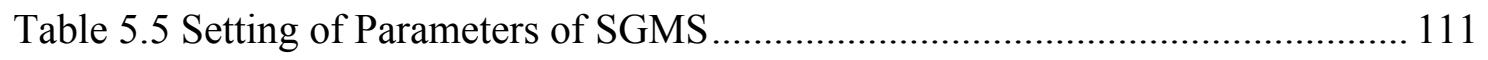

Table 5.6 Comparison of Computational Cost of Initial Authentication.................... 111

Table 5.7 Comparison of Energy Cost of Fast Authentication in Handover Phase..... 112 


\section{Chapter 1 Introduction}

With the rapid development of computer networks, electronic technology, and control technology as well as increased requirements for intelligence in modern industry, the need for physical devices to become more compatible with information and the Internet is growing fast [1]. However, there is still a serious gap between the cyber world, where information is exchanged and transformed, and the physical world in which we live [2]. The cyber world and the physical world have been considered as two different entities in the past decades. In line with this issue, the cyber-physical system (CPS), which can seamlessly integrate the functions of computing, communication, and control, has developed as an intelligent system that performs computing and physical processes as a single entity. A CPS mainly consists of two subsystems, a physical processing system and a cyber system, which are usually composed of a set of networked agents including sensors, actuators, control processing units, and communication devices, as shown in Figure 1.1 [3]. The physical process system is controlled and monitored by the cyber system, which consists of small

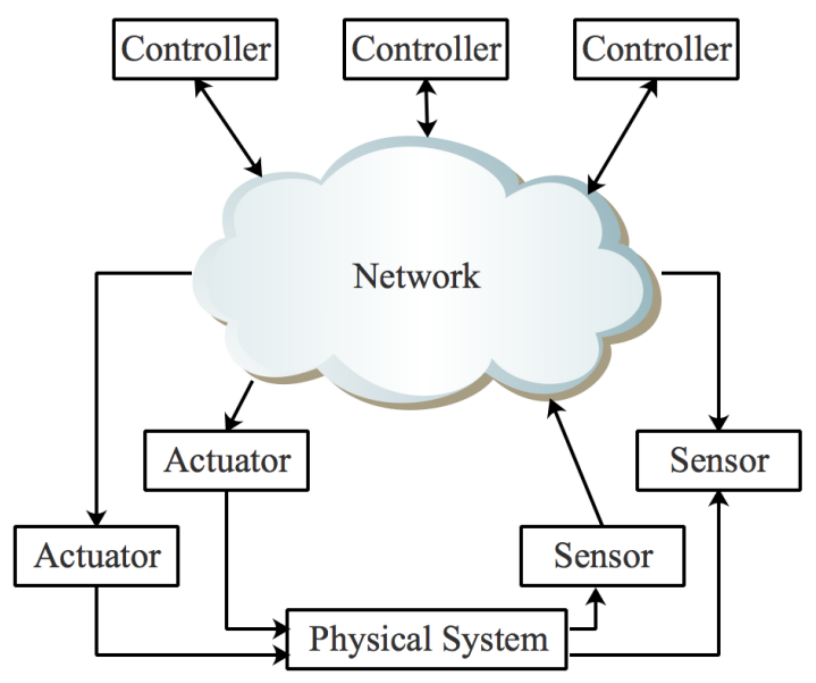

Figure 1.1 General Architecture of a CPS 
devices with basic computation and wireless communication capabilities. While functioning, all the units within the system cooperate with each other over the CPS network, which links the cyber system and the physical system, forming a large-scale heterogeneous distributed real-time system.

Machine-to-machine (M2M) communication, which allows wireless and wired systems to monitor different environmental and physical conditions without human intervention, is the major communication technology used in most existing CPSs. In M2M, each device, such as a sensor or a smart meter, captures an event as it happens, such as a change of temperature or inventory level, which will be relayed through a wireless, wired, or hybrid network to an application that translates the captured event into meaningful information to trigger an actuation [4].

Since M2M devices are linked and managed through communication technologies that require integration and convergence among many different communication systems, architectures for $\mathrm{M} 2 \mathrm{M}$ have been proposed and developed in various standards organizations. The European Telecommunications Standards Institute (ETSI) has formed a technical committee (TC) to perform the standardization work on M2M communication. The main goal of the committee is to develop an end-to-end (E2E) architecture for M2M systems with outstanding features such as sensor network integration, quality of service (QoS), security, discovery and localization, and so on [5]. Figure 1.2 shows the high-level architecture of the M2M communications paradigm, which mainly consists of two domains: (1) the M2M device and gateway domain and (2) the network domain.

In the M2M device and gateway domain, the M2M devices can collect monitoring data from different sensors and transmit the gathered information or reply to the requests for the information automatically. The M2M devices can be connected to the network domain via an access network or an M2M gateway, which acts as a proxy between the M2M devices and the network domain. An M2M area network can provide the connectivity between the M2M devices and the M2M gateways. The implementation of the M2M area networks will rely on wireless personal area network 


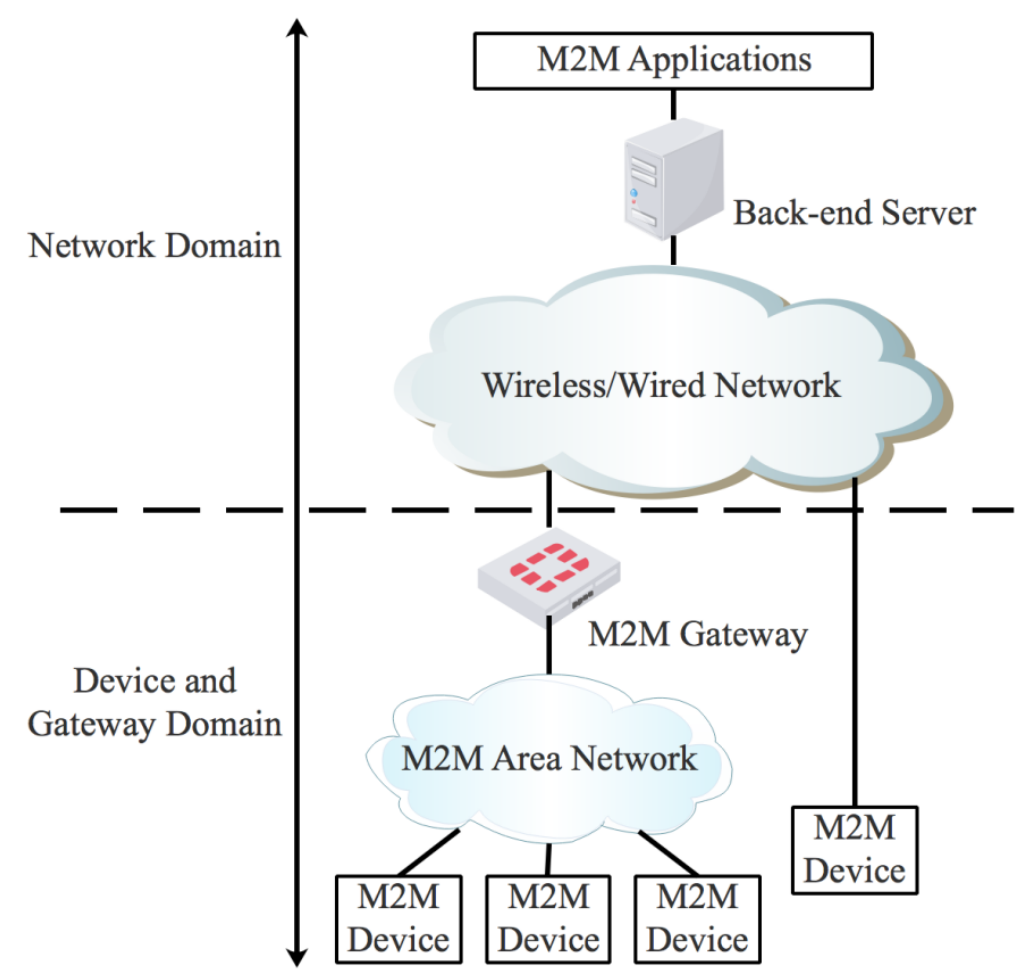

Figure 1.2 High-Level Architecture for M2M

technologies such as Zigbee, Bluetooth, and so on, or other local networks such as Power Line Communications (PLC), Meter-Bus (M-BUS), Wireless M-BUS [6].

The major function of the M2M network domain is to provide reliable wired or wireless channels to enable transmission of sensory data from the M2M device and gateway domain to M2M applications. An access network, such as xDSL (Digital Subscriber Line Technologies), satellite, global system for mobile communications (GSM) enhanced data rates for GSM evolution (EDGE) radio access network (GERAN), wireless local area network (W-LAN), and worldwide interoperability for microwave access (WiMAX), can be implemented to allow the M2M devices or gateways to communicate with the core network (CN) [7]. The M2M CN provides the Internet Protocol (IP) connectivity, services and network control functions, roaming, and interconnection with other networks. The M2M service capabilities (SCs) provide 
the M2M functions, which are exposed through a set of open interfaces. The M2M applications can use the M2M SCs through these interfaces.

However, with an enormous growth in resource constrained devices emerging in the M2M network, the integration of those devices implemented with various wireless personal area network technologies and the IP-based services seem to become more challenging. In order to allow a large number of devices to interoperate with the Internet for extending the use of M2M applications, Internet Protocol version 6 (IPv6) over low-power wireless personal area networks (LoWPANs) (6LoWPANs) [8] proposed by the Internet Engineering Task Force (IETF) could be applied to the M2M system. By 6LoWPAN, the IPv6 is adapted to be applicable to wireless embedded devices which enables the seamless connection between the open Internet and the M2M area network. It is foreseeable that sensor nodes, in combination with the nextgeneration Internet protocol IPv6, will accelerate the development of the M2M technology.

\subsection{LoWPAN}

Due to a large number of devices, the M2M needs to achieve the networking of devices and the compatibility and interoperability among different types of equipment. IPv6, which enables more ubiquitous computation, is a better choice for M2M sensing applications because it has much larger address space. However, the Institute of Electrical and Electronics Engineers (IEEE) 802.15.4 [9] only specified the standard of two bottom layers for low-rate wireless personal area networks. Other higher-level layers and interoperability sublayers are not defined in IEEE 802.15.4. How to integrate IPv6 with the existing M2M network has been a hot issue. Therefore, the IETF has been developing the 6LoWPAN standard which optimizes IPv6 for use with low-power, low-bandwidth communication technologies [10].

The IETF has chartered the 6LoWPAN working group to standardize protocols at various layers to connect the LoWPANs to the open Internet. The 6LoWPAN standard adopts two bottom layers of IEEE 802.15.4 [11]. However, the packet length supported by 6LoWPAN bottom layers is 127 bytes while it is at least 1280 bytes in IPv6. In 
order to connect seamlessly between the media access control ( $\left.\mathrm{MAC}^{1}\right)$ layer and the network layer, the 6LoWPAN working group suggests that adding an adaptation layer between these two layers to realize the IPv6 header compression, packet fragmentation, reassembly and mesh route forwarding. Figure 1.3 is the reference model of 6LoWPAN protocol stack.

\begin{tabular}{|c|}
\hline Application Layer \\
\hline Transport Layer \\
\hline Network Layer \\
\hline 6LoWPAN Adaptation Layer \\
\hline IEEE 802.15.4 Media Access Control Layer \\
\hline IEEE 802.15.4 Physical Layer \\
\hline
\end{tabular}

Figure 1.3 The Reference Model of 6LoWPAN Protocol Stack

\subsubsection{LoWPAN Architecture}

A 6LoWPAN network, which consists of numerous low-cost devices connected using wireless links, can be deployed in real-world applications with limited resources requirements. A simple example of how to deploy a 6LoWPAN network is presented in Figure 1.4. As shown in the figure, the 6LoWPAN networks and several common devices, such as servers, computers, printers, etc., are connected to the Internet through the IP routers. There are three types of devices inside a typical LoWPAN: hosts, routers and one or more edge routers.

6LoWPAN host (6LH): Hosts are end devices which do not have the capability to route packets to other communicating devices in the network. As being restricted by the characteristics of 6LoWPAN devices (i.e. low power, low memory and low processing), battery-operated hosts may sleep periodically to conserve energy. Mobile hosts can move freely within a LoWPAN or between LoWPANs.

6LoWPAN router (6LR): Routers are essential intermediate nodes which are 


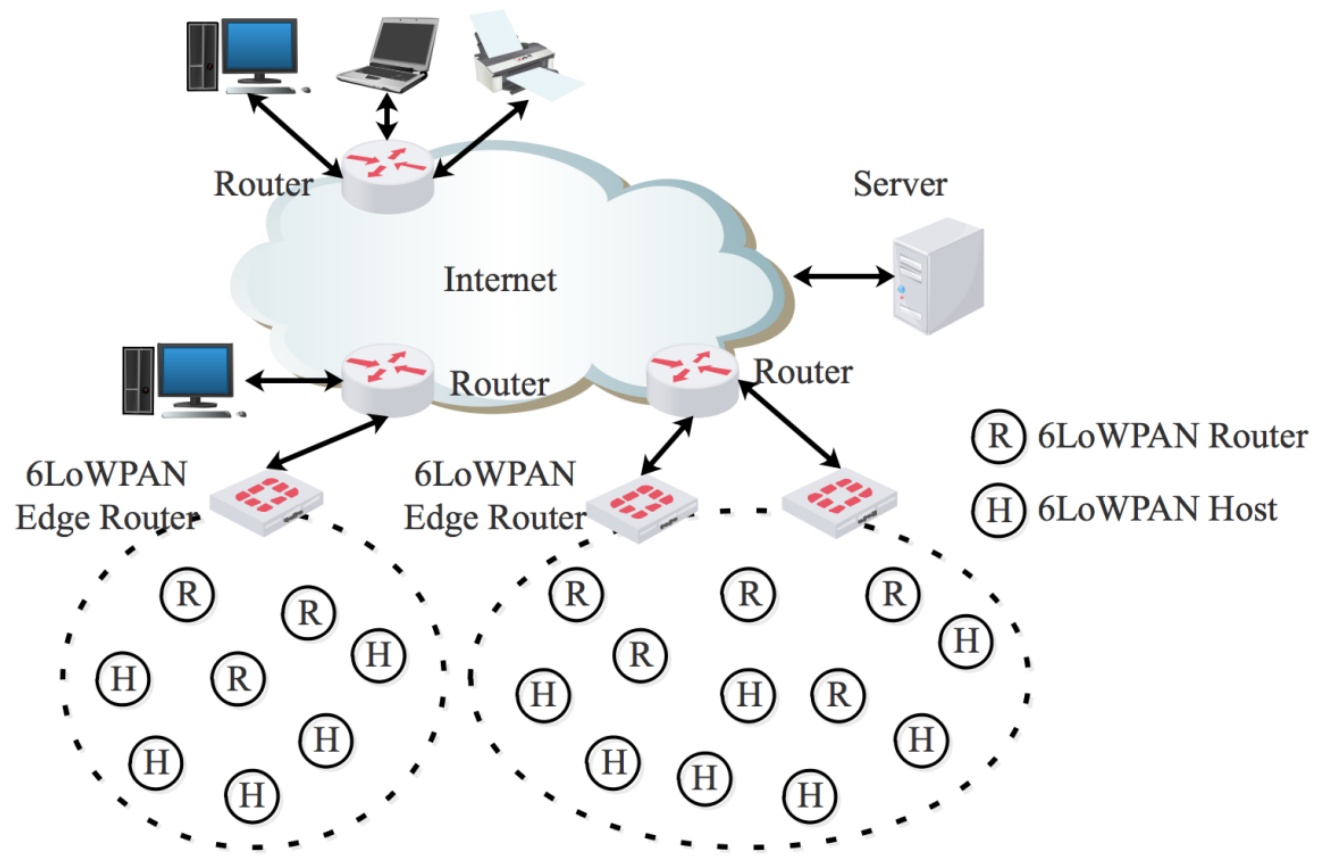

Figure 1.4 The 6LoWPAN Architecture

responsible for forwarding and routing data packets transmitted between the 6LoWPAN hosts and the edge router. Routers commonly are placed in the multi-hop mesh topology of 6LoWPAN networks.

6LoWPAN edge router (6LER): The 6LER plays a significant part as it connects the 6LoWPAN network and the backbone IPv6 network. In specific, for the data transmission between different 6LoWPANs, the 6LER is responsible for relaying the data packets. Moreover, for data packets originated from backbone network and destined to the 6LoWPAN network, the 6LER applies 6LoWPAN IPv6 header compression and packet fragmentation to transform the data into the format fit for the 6LoWPAN network.

\subsubsection{LoWPAN Applications}

Although the applications of 6LoWPAN are restricted by the inherent characteristics, i.e. low power, short range, low bit rate [12], there are a wide variety of 
applications based on 6LoWPAN, with the request of system flexibility without cumbersome cables and high energy consumption, that could benefit the world [13].

As one potential application, the ubiquitous healthcare service enables remote monitoring health status of patients in real time. Several 6LoWPAN based healthcare systems, such as those in $[14,15]$, have been proposed to offer efficient, flexible and reliable services for monitoring and tracking patients, staffs, and even bio-devices. The integration of the IP technology in low-power wireless devices can achieve real-time communications, allowing that the healthcare specialists diagnose the disease by the information of the patients received from remotely controlled wearable sensors via the Internet.

Another possible application is to monitor the manufacturing process in industry $[16,17]$, where diverse sensors, actuators and controllers are connected to achieve passive monitoring and active control and automation. The 6LoWPAN protocol optimizes the traditional IP techniques to enable efficient transmission of IPv6 packets over the wireless networks. The seamless integration of low power wireless devices into the hierarchical industrial IP networks allows them to interoperate with the rest of IPv6 enabled Internet, which largely promotes the M2M.

Moreover, 6LoWPAN can be applied in the home automation control system [18, 19] to offer services such as monitoring home temperature and status of doors or windows, as well as controlling the air conditioner or switches. Telematics $[20,21]$ can also be achieved by using 6LoWPAN nodes for monitoring cars' movements. In addition to the applications mentioned above, a wide range of applications (i.e. agricultural monitoring, asset management, smart office, etc.) can be enabled by the 6LoWPAN to realize IP connectivity.

\subsubsection{Mobility Protocols in 6LoWPAN}

Mobility is the act that the point of attachment of a mobile node (MN) changes which is caused by some reasons such as physical movement, failure of some routers or radio channel conditions [22]. However, changing the point of attachment may result in longer handover delay, packet loss or even have an influence on the proper functioning 
of applications. If an MN moves to a new domain with a different IP address assigned, this node will lose its previous connections, but if the previous IP address remains unchanged, the data packet cannot be correctly routed since the latest point of attachment cannot be reflected by the fixed address. Thus, a mobility protocol which supports efficient handovers for $\mathrm{MNs}$ is urgently needed to deal with the problems encountered during the handover.

- $\quad$ Mobile IPv6 (MIPv6) [23]: MIPv6 is a host-based protocol proposed for MNs to continuously access the network services while moving among foreign networks. It is required that the $\mathrm{MN}$ needs to send mobility-related signaling messages to its home agent (HA) to maintain the binding state between the MN's care-of address and its home address. MIPv6 ensures that the MN can keep its home address while roaming in the network. The home address is regarded as an identification of an $\mathrm{MN}$, irrespective of the node's current location. As for the care-of address which associates the node's home address, it will be updated each time when $\mathrm{MN}$ attaches to a new foreign agent (FA). Therefore, the data packets can be accurately redirected to the $\mathrm{MN}$ based on its care-of address. Subsequent work [24, 25] has been proposed to improve the performance of MIPv6. However, protocols based on MIPv6 require that the mobility-related signaling needs to be sent by the node itself which is not applicable for resource constrained sensor nodes.

- Proxy Mobile IPv6 (PMIPv6) [26]: PMIPv6 is a network-based localized mobility management protocol proposed by the IETF network-based localized mobility management (NETLMM) working group, to support mobility without the involvement of the MN. Unlike MIPv6, the mobile access gateway (MAG), which the $\mathrm{MN}$ is attached to, is responsible for exchanging the signaling related to mobility with the local mobility anchor (LMA) on behalf of the MN. PMIPv6 allows the MN to maintain the same network prefix assigned by the LMA even when it is away from its home network. Hence, the configuration of care-of address of the MN is not needed, so that PMIPv6 
significantly reduces the signaling overhead of the $\mathrm{MN}$ and lower the handoff latency.

- Network Mobility (NEMO) [27]: Evolved from the MIPv6, NEMO is a network-based mobility protocol which supports the mobility of an entire network viewed as a single unit. A mobile router (MR) is employed to connect the mobile network and the Internet. As the default gateway of the mobile network, MR is capable of changing the point of the attachment of the entire mobile network. In common with the MIPv6, NEMO also requires both the home address and the care-of address. The MR is responsible for registering a unique home address with the HA and configuring a care-of address used for forwarding the data packets when moving away. The benefit is that NEMO also provides reachability for local fixed nodes which regard the mobile network as a common IPv6 access network.

The comparison among the MIPv6, the PMIPv6 and the NEMO is presented in Table 1.1. The MNs implemented the MIPv6 need to perform all the mobility signaling with the HA themselves which is a huge consumption of resources. By the NEMO, the MR can perform the MIPv6 functions on behalf of the nodes within its network. Although the signaling cost is less than the MIPv6, a powerful MR is still required. As for PMIPv6, actions with high computational overhead are migrated to the MAG instead of the MNs. Besides, the MIPv6 and the NEMO adopt the Internet Protocol Security (IPsec) to provide the protection of the signaling between the MN/MR and the

Table 1.1 Comparison of Mobility Protocols

\begin{tabular}{ccccc}
\hline \hline & $\begin{array}{c}\text { Node/ Network } \\
\text { Mobility }\end{array}$ & $\begin{array}{c}\text { Node/ Network- } \\
\text { based Mobility }\end{array}$ & Care-of Address & Data Buffered \\
\hline MIPv6 & Node & Node-based & Required & HA \\
PMIPv6 & Node & Network-based & Not Required & LMA or MAG \\
NEMO & Network & Network-based & Required & HA \\
\hline \hline
\end{tabular}


HA which is not suitable for resource constrained sensor nodes. In PMIPv6, the PMIPv6 standard only recommends that the transmission of the signaling between the MAG and the LMA should be secured by the IPsec while the protection for the messages sent from the MNs is not specified. Since the 6LoWPAN devices cannot support the full functions of the IPsec due to the unaffordable energy cost, it is possible to implement PMIPv6 as a mobility support protocol in 6LoWPAN.

\subsubsection{Security of 6LoWPAN Networks}

Although the mobility of the 6LoWPAN devices brings the flexibility of a system construction, it also may lead to more security challenges to the whole system. The 6LoWPAN communication networks have several insecure features. First, a 6LoWPAN network is easily eavesdropped due to the broadcast nature of the communication medium. Second, sensor nodes are easy to be compromised by malicious attacks due to limited energy capabilities and computing resources for security services. In addition, traditional wired based security schemes cannot be straightly applied in 6LoWPAN network since the computational overhead of those schemes are too much for the resource constrained 6LoWPAN devices. The above mentioned insecure features make the 6LoWPAN system vulnerable to malicious adversaries. There is a high demand for exploring solutions to effectively protect 6LoWPAN communications.

To establish a secure 6LoWPAN communication, security mechanisms should meet the following requirements [28-30]:

- Data Confidentiality: Confidentiality prevents private sensory data from being eavesdropped by passive attackers, which can be achieved by using authentication and cryptographic encryption to ensure that the information transmitted in the 6LoWPAN networks can be accessible only to authorized parties.

- Data Integrity: Data integrity ensures that the data cannot be illegally altered in transit by unauthorized participants before reaching the right recipient. Integrity, which can be achieved by employing cryptographic integrity checks, 
is critical in 6LoWPAN networks since tampering with the information could result in severe consequences.

- Data Authentication: Though integrity guarantees that the data cannot be illegally altered in a transmission, it cannot know whether the data is originated from the recipient which is authorized to access the private information. As a prerequisite for secure communications, authentication allows the authenticator to identify whether the data source is sent from a reliable party.

- Data freshness: Data freshness is that the data as well as the secret keys are generated recently and have not been replayed by any adversary.

- Availability: Since wireless radio communication is often used in 6LoWPAN networks, communication availability can be affected by jamming attacks. To defend such attacks, a mechanism should be applied in the system to provide availability, which ensures that the network services should always be available for the authorized parties.

- Robustness: Robustness ensures that even some exceptions occurred, such as malicious attacks on networks or equipment failures, the networks should still be usable.

- Resiliency: An acceptable level of security of the system can be maintained even when some 6LoWPAN devices are compromised, which prevent the attackers from taking control of the system.

- Energy efficiency: Since most of the 6LoWPAN nodes are devices with constrained power capacities, limited computing and storage resources, the mechanisms used in the 6LoWPAN networks should be lightweight to prolong the lifetime of the network.

- Assurance: Assurance is the ability to ensure that different information should be disseminated at specified guaranteed level.

The security requirements for 6LoWPAN mentioned above should be achieved to overcome vulnerabilities to various security threats, which have been specified by [2831]. The resource-constrained 6LoWPAN devices are usually placed in unattended and 
accessible locations without specific supervision and are more easily tampered with by unauthorized people compared with traditional equipment that has enough resources and is carefully protected. Thus, the security issues faced by the 6LoWPAN system are challenging. In addition to the security issues encountered in general scenarios, the following security threats classified in a layer-wise order should be considered in 6LoWPAN communication.

- Physical Layer [13]: An attacker is able to physically move a 6LoWPAN device from an authorized place or even destroy the devices permanently. The shared secrets, credentials and keys stored inside a device could be stolen and modified to allow an adversary to inject malicious packets or clone many legitimate nodes. Moreover, denial of service (DoS) attacks at the physical layer could be jamming or tampering the signal. However, these attacks are difficult to detect due to the unattended nature of 6LoWPAN.

- MAC $^{1}$ Layer: Being characterized as low-power and low-cost devices, 6LoWPAN devices always require resources saving operations, such as sleep, to extend their lifetime. Such constraints can be exploited by attackers to drain the power of the 6LoWPAN devices with many unnecessary tasks. In addition, packet collision launched by the attackers can result in disrupting of services or even battery exhaustion. Packet collision, which only consumes little energy while it is hard to detect, can cause packet loss.

- Network layer: In addition to the common malicious attacks against network layer such as selective forwarding, sinkhole attack, wormhole attack and Sybil attack, which disrupt the network services, security attacks at the adaptation layer should be taken seriously as well. Since the 6LoWPAN devices cannot support large IPv6 packets from the outside network, the packets are fragmented if the payload length exceeds the maximum number of bytes. The datagram size or offset of the fragmented packets may be modified during the transmission. Attackers can inject malicious fragments to prevent the nodes from extracting the correct information. Moreover, the reassembly buffer 
space could be quickly occupied if only parts of the fragments are sent by the adversaries.

- Transport layer: At this layer, attackers could launch Transmission Control Protocol (TCP) synchronize (SYN) message flood attack to consume considerable resources of a legitimate device by forcing a host to retain enough resource for these half-open connections and request retransmissions of the frames. Besides, security concerns at this layer raised in [32] that the overload of the User Datagram Protocol (UDP) ports may result in incorrect types of payload or the wrong content. To solve the problem, security mechanisms that provide content validation and integrity should be employed when using those ports.

- Application layer: An adversary may launch overwhelm attack by deceptive sensory stimuli to cause large volumes of traffic forwarded to the edge router. Confidential application data may be illegal copied or forged to prevent the system from operating properly.

As well as the above mentioned malicious attacks classified in a layer-wise order, privacy attacks require attentions, including monitoring/eavesdropping data transmitted in the network by other devices, revealing nodes' identities or other confidential data to unauthorized third parties. A privacy concern of interface identifiers (IIDs) in 6LoWPAN is pointed out by [33] that there is no protection method for the global uniqueness of 6LoWPAN IIDs derived from 64 bits extended unique identifier (EUI) $\mathrm{MAC}^{1}$ addresses. Another privacy concern raised by [34] is that the generation of IIDs with insufficient entropy may pose security threats to the 6LoWPAN devices such as location tracking, device-specific vulnerability exploitation and address scanning. The vulnerabilities of the 6LoWPAN networks will be exhibited under all of the above mentioned malicious attacks. The potential damages caused by the compromised 6LoWPAN networks would hinder the adoption of the technique in practical. Therefore, the security schemes which could protect the 6LoWPAN networks are required. 


\subsection{Motivation}

Unlike traditional IPv6 network, 6LoWPAN devices are characterized as resourcelimited devices in terms of power, storage, memory and battery life. Due to these constraints, traditional and mature security mechanisms cannot be directly applied to the 6LoWPAN network. A primary design criterion for 6LoWPAN systems is transmitting a message over the network as small as possible since the energy cost of packets transmission is much higher than local computation [28]. When taking the inherent properties of 6LoWPAN devices into consideration, a secure 6LoWPAN system design is based on a tradeoff between efficiency and security.

Attacks in 6LoWPAN networks from an attacker outside the network can be classified as passive and active attacks. For example, at the physical layer, passive attacks such as eavesdropping and traffic analysis are hard to detect while active attacks such as DoS attacks and scrambling attacks are easy to identify but could cause much more damage than passive attacks. Corresponding detection and authentication mechanisms should be in place. As for insider attacks, aiming at preventing the system from working normally, an adversary could compromise a legitimate device to extract confidential information to launch destructive attacks. How to protect the 6LoWPAN nodes against node capture attack is very challenging.

As the sensory information collected by the 6LoWPAN devices may be sensitive, certain protection measures for preventing the attacker from deriving privacy information, such as encryption or access control, should be taken. In the context of 6LoWPAN networks, identities, locations, and sensory information can all be considered as privacy.

The vulnerabilities of the 6LoWPAN system may lead to the system to be destroyed under attacks mentioned above. It is vital to construct secure and efficient 6LoWPAN communication systems with strong countermeasures against various malicious attacks [35]. 


\subsection{Objectives}

The security mechanisms of 6LoWPAN networks should be enhanced to provide confidentiality, integrity and availability for secure communications with the ability to prevent hostile attacks. The objectives of this thesis are listed below.

- The 6LoWPAN devices can obtain sensory data or gather other information and send them out to the 6LoWPAN gateway or servers. Since the resource constrained 6LoWPAN devices are usually deployed in unattended and accessible places, they are more easily tampered by attackers to cause damages or take illegal actions. Thus, authentication mechanisms are required to prevent against eavesdropping or unauthorized accesses to the data while minimizing the cost of resources. These mechanisms should also provide mobility support for 6LoWPAN nodes to enable secure and efficient handovers.

- To overcome the threats to steal or to alter the information delivered in the communication networks, proper encryption method should be adopted to ensure fast verification of the messages, and integrity and confidentiality of data communication among 6LoWPAN devices.

- The back-end authentication server should guarantee that its services are accessible to authorized devices at any time. Servers may help devices establish shared secret keys by distributing keying materials. However, key management is not defined in the 6LoWPAN specification. Therefore, appropriate key management methods should be developed to regulate the secret key distribution and provide fast key generation approach for mobile 6LoWPAN devices.

\subsection{Contributions of the Thesis}

The research in this thesis focuses on developing authentication and key establishment protocols to deal with the challenging security and mobility issues in the 
6LoWPAN networks. The main contributions of the research work presented in this thesis are listed below.

- To provide security protection for mobile 6LoWPAN hosts, an enhanced authentication and key establishment scheme in 6LoWPAN (EAKES6Lo) is proposed for secure communications between the hosts and the gateway. With the help of the handover ticket issued by the server and assigned to each legitimate mobile host, the authentication procedure occurred during each handover is simplified while still meeting the security requirements and as a result the local computational overhead and the energy cost of transmission are apparently reduced.

- $\quad$ To further improve the handover efficiency, an efficient and secure PMIPv6based handover (ESPH) scheme is proposed. PMIPv6 is a network-based mobility protocol which is applicable for resource constrained 6LoWPAN devices since a proxy exchanges the mobility-related messages on behalf of the MNs. The session key established between the $\mathrm{MN}$ and the gateway is based on a hash chain generated according to the movement of the device. Thus, the handover latency and the packet loss problems are further reduced.

- To provide security protections for seamless group handovers, a secure group mobility scheme (SGMS) is designed to meet the requirement of the future Internet of Things (IoT) with supporting a large number of devices as a prerequisite. The formal verification by the verification tools and the performance evaluation results show that the proposed scheme could not only enhance the security functionalities with the ability to prevent various malicious attacks but also be computationally efficient.

\subsection{Organization of the Thesis}

The remainder of this thesis is organized as follows. In Chapter 2, the current research works on the security of the 6LoWPAN, which are categorized as detection, authentication, key management, privacy and the compression of the IPsec solutions, are reviewed. In Chapter 3, an authentication and key establishment scheme to enhance 
security in 6LoWPAN (EAKES6Lo) is described in details. In Chapter 4, an efficient and secure PMIPv6-based handover (ESPH) scheme is proposed. In Chapter 5, a secure group mobility scheme (SGMS) is proposed to provide security and fast handover support for groups of 6LoWPAN MNs. Finally, the conclusions and the future work which could improve the security for 6LoWPAN networks are discussed in Chapter 6. 


\section{Chapter 2 Related Works}

Security is a major concern of most 6LoWPAN applications, with a high demand for appropriate security mechanisms to protect the 6LoWPAN communication systems. Many solutions applied in the 6LoWPAN networks are available to overcome the vulnerabilities caused by the security threats mentioned earlier. In this chapter, the solutions that are currently available and used in the 6LoWPAN networks will be introduced. These security solutions can be classified as detection, authentication, key management, privacy and the compression of the IPsec schemes.

\subsection{Detection Schemes}

Sensor nodes are generally deployed and distributed in the unattended places, and it is easy for messages to be attacked in the transmission process. As DoS attacks are considered to be an important security issue, a novel DoS detection architecture that integrates an intrusion detection system (IDS) [36] is proposed. The network framework of this proposal is developed within the project aimed to realize the integration of the IoT and the mainstream enterprise systems and provide support for E2E business applications. To evaluate the performance of the proposed architecture, a preliminary implementation was completed and tested against a real DoS attack using a penetration testing system. The result proves that their detection mechanism is successful in detecting DoS attacks on 6LoWPAN. In future, the implementation of this architecture is expected to be completed and tested against different real attacks.

In addition to DoS attack, wormhole attack is one of the most common security attacks in a sensor network that a malicious node tunnels the collected data to another malicious node placed at a distant location, which can significantly disrupt the routing protocols but it is hard to detect. Krentz et al. [37] proposed a secure channel reciprocity-based wormhole detection (SCREWED) mechanism to detect hidden wormholes based on the received signal strength indicators (RSSIs). SCREWED contains two phases, namely sampling and judgement. In the sampling phase, the 
RSSIs of the two designated frames PING and PONG, which are sent with random transmission powers through different channels, are recorded for further analysis. In the judgement phase, whether a wormhole node exists or not is determined by the number of received PONGs and the sample correlation coefficient. Unlike SCREWED, another wormhole detection mechanism proposed by Gai [38] is based on the rank value of each node. Two attributes are used to discover whether a source node sending messages is malicious. One attribute is Rank_Threshold which is the difference of the rank values between its parent and the node itself. Another attribute is Rank_Diff which is the rank difference between the source node and the node itself. If the Rank_Diff is greater than the Rank_Threshold, the source node is regarded as an attacker. This detection system is easy to be implemented without any complex computation.

As another destructive attack against routing, sinkhole attack aims to attract a large amount of data packets by falsely claiming that it has the optimum route. An IDS of sinkhole attacks on 6LoWPAN for IoT (INTI) [39] is proposed to identify sinkhole attacks in 6LoWPAN network taking into account the mobility of the nodes. Three predictions: uncertainty $(u)$, belief $(b)$ and disbelief $(d)$ are used in the calculation of a node's reputation computed by $\operatorname{Beta}(\alpha, \beta)$ distribution. If the reputation result is less than 0.5 , the node is assumed as a sinkhole node which will be isolated from the network. The performance evaluation shows that the detection rate is $92 \%$ if the nodes are fixed. And the rate becomes $75 \%$ if the simulation is conducted in the mobile scenario.

The above-mentioned detection mechanisms mainly target at one particular attack. To extend the IDS to detect multiple attacks at the same time, an IDS called SVELTE [40] which literary means elegantly slim is proposed by Raza et al. to protect against multiple attacks in a 6LoWPAN network. There are three main modules in the SVELTE which are a 6LoWPAN mapper, an intrusion detection component and a distributed mini-firewall. The 6LoWPAN mapper gathers information about the routing protocol for low-power and lossy networks (RPL) and reconstructs the RPL topology in the edge router. Four detection algorithms implemented in the intrusion detection component are used for detecting and correcting the inconsistencies of RPL topology, 
checking node availability, validating the routing graph and verifying the E2E packet loss. This distributed mini-firewall is proposed to protect the 6LoWPAN network against external hosts if the number of reports accusing those hosts reaches the specified threshold. This proposed detection system could easily be extended to detect many potential 6LoWPAN intrusions.

Since many 6LoWPAN devices are simple and low cost, attackers can launch various attacks to compromise the functionality of those devices and obtain their credentials or secret keys. Detection mechanisms are needed to early detect malicious attacks or even forecast those attacks before any severe damage of the network is caused. In contrast to attacks which are easy to be detected, attacks like eavesdropping and traffic analysis which are difficult to be noticed should be prevented as well. Due to resource constraints on a number of 6LoWPAN devices, detection mechanisms should be lightweight and easy to be expanded.

\subsection{Authentication Schemes}

To support the secure connections between the legitimate devices and the server, different authentication approaches have been proposed to support security functionalities and try to meet the security requirements of communication systems based on 6LoWPAN. Authentication procedures can ensure the information transmitted to the legitimate users and prevent the systems from compromised attacks.

Wang et al. [41] proposed a secure mobility support scheme for 6LoWPAN based on its novel hierarchical IPv6 address structure which consists of three parts: the global routing prefix, the full-function device (FFD) ID and the reduced-function device (RFD) ID. With this address structure, the security of intra-network and inter-network mobility handover can be achieved by the authentication process. The performance evaluation demonstrates that the mobility handover cost and delay are reduced. However, how to pre-distribute the pairwise key between every two nodes is not describe in this paper.

A secure authentication and key establishment scheme (SAKES) [42] for M2M communication in the 6LoWPAN is proposed. SAKES uses pairwise keys during node 
authentication phase and the lightweight asymmetric key cryptography during the session key establishment process. Security analysis is performed for different attack scenarios. The impact of DoS attack on the performance of the 6LoWPAN is evaluated. The evaluation is carried out by using the OPNET simulation tool. Their simulation result shows the severity of DoS attack and the necessity of the proposed security scheme so as to maintain the constrained resources. However, it has a limitation that the security protection has only provided to the static nodes in the 6LoWPAN networks and the attacks can only be prevented under the assumption that the 6LHs and the 6LRs have not been compromised.

Oliveira et al. [43] proposed a network admission control solution for 6LoWPAN networks aimed at isolating unauthorized nodes from building connections with the legitimate nodes or with the Internet. Before a device is deployed in the target place, it should be manually configured with pre-shared keys using wired connection. Next, the presence of a node can be accurately detected by the edge router after a minimized neighbor discovery protocol. Then, the authenticity of a detected node is verified using a one-way challenge authentication process. The authorized node list is sent to legitimate nodes periodically or once the authorization list is updated. After the authentication of a node, the node starts sending and receiving data packets. The acceptance of the incoming frames is determined by whether the source and the destination addresses of those frames belong to an authorized node.

As the size of a data packet supported by the network layer and the bottom layer is different, packets fragmentation is provided at the adaptation layer to enable large IPv6 packets to be transmitted over the 6LoWPAN network. However, due to the short of the authentication mechanisms for fragments, duplicate or malicious fragments injected on the sly are hard to be detected. Besides, the memory of a node can be occupied with incomplete packets sent by the attackers, which completely blocks the node from receiving any valid fragmented packet. To fight against these attacks, Hummen et al. [44] proposed two lightweight schemes for resource constrained 6LoWPAN networks. The content-chaining scheme appends a token after the payload of each fragmented packet. The token, which is a hash value of the payload and the token from the next 
packet, will be verified by the destination node. Moreover, the split buffer approach is proposed to allow nodes to discard the incomplete packet with the lowest score, which is rated based on the sending behavior for a packet.

As a potential mobility support protocol for IPv6, PMIPv6 is suitable to be implemented in the 6LoWPAN network due to its low signaling overhead. Certain security protection mechanisms are needed for PMIPv6 since it is very sensitive to security threats. Zhou et al. [45] proposed an authentication method in PMIPv6 to provide access authentication on MNs as well as prevent against some common security threats. The performance analysis is conducted with the consideration of the impacts of parameters such as mobility and traffic. However, when the MN changes its attached point, it still needs to send messages to the previous MAG (PMAG) which may not be applicable in some scenarios that the node has already lost the connection with the PMAG while moving. A secure password authentication mechanism (SPAM) for secured seamless handover in PMIPv6 has been proposed in [46]. Though its computational overhead is low, SPAM has some security weaknesses as pointed out in [47]. The nonce sent in the first message during authentication phase cannot be identified whether it is fresh or not. Besides, if the group key shared among MAGs and the LMA is exploited by a dishonest entity, some confidential information could be easily obtained to register many legal but not existed MNs, which may lead to DoS attack. A secure fast handover mechanism for PMIPv6 networks has been proposed in [48] to lower the authentication delay and provide security functionality in the handover process. It suffers from replay attacks and impersonation attacks.

\subsection{Key Management Schemes}

Key management is the management of various cryptographic keys in a cryptosystem, which includes dealing with the generation, exchange, storage, use, and replacement of different keys.

Krentz et al. [49] proposed an adaptable pairwise key establishment scheme (APKES) to realize energy efficiency and DoS resilience. APKES is more like a framework that four pluggable schemes can be integrated with the framework so that 
the whole scheme can be applied to different 6LoWPAN networks. Three phases are involved in APKES: 1) Optional preloading of short addresses: The 802.15.4 addresses which consist of a 2-byte personal area network (PAN) ID and a 2-byte short or a 8byte EUI-64 are used as devices' ID. 2) Preloading of cryptographic material: the cryptographic materials should be preloaded on a device before it is placed in the network. 3) Pairwise key establishment: three newly defined 802.15.4 commands are employed during the three-way message exchange to establish pairwise keys between devices. However, comprised devices could still be threats since the secret pairwise key can be learned by the attacker.

Misra et al. [17] proposed an adapted public key infrastructure (PKI) model to provide an efficient and secure integration into industrial 6LoWPAN networks. To relieve the nodes of processing overhead, the bulk of the key-related actions are migrated to the edge routers. Only two keys need to be saved permanently in the nodes' memory, which are the private key of the node and the public key of the 6LER. If a node requires the public key of a certain node, a request is sent to the edge router. Once the node receives the key from the edge router, it can start communicating with the corresponding device. The evaluation results show that the proposed PKI model can be implemented efficiently and safely in industrial 6LoWPAN devices.

Key management is one of the greatest challenges in 6LoWPAN network security. Symmetric key based key management mechanisms are more popular than asymmetric key-based schemes due to lower computation cost. With a large variety of devices deployed in the 6LoWPAN system, the group key management and hybrid key management, which combines symmetric and asymmetric cryptography, are possible future trends and should be addressed.

\subsection{Privacy Schemes}

In most existing 6LoWPAN address configuration protocols, the IP address assigned to an MN remains the same even when it is away from home. Therefore, it is easy for eavesdroppers to track the node through its permanent IP address. To this end, a 6LoWPAN addressing scheme with privacy support is proposed in [50] to lessen the 
privacy concerns of a permanent IP address by periodically changing the address even without duplicate address detection (DAD) and extra overheads. A node's IP address is randomly chosen from an address set with randomly generated size. Therefore, the eavesdropper cannot infer a specified node from different addresses. The evaluation results show that this scheme achieves the address privacy with better performance.

As for most current address configuration solutions with $\mathrm{DAD}$, a complete address constructed by a newly joined node is broadcasted in the network. However, if an attacker seizes the address and registers it in advance, the node's address becomes invalid. Thus, the legitimate node may not function properly in the 6LoWPAN network since the attacker keeps claiming that the node's addresses intended to be configured are occupied. In order to protect the address from being in possession of the attackers, an improved DAD for 6LoWPAN [51] is proposed. To keep the privacy of the address, the node will only broadcast a part of the address rather than the exposure of a complete address in the improved DAD so that the attacker is unable to occupy the target address. Besides, broadcasting a partial address in the improved DAD not only improves the success rate in address registration but also lowers the transmission cost.

\subsection{The Compression of the IPsec Schemes}

With the help of the 6LoWPAN, numerous smart devices implemented with various communication technologies will be interconnected together to achieve all-IP network. To protect the data transmission over insecure channels, E2E security should be provided with certain security mechanisms. However, E2E security is not provided in IEEE 802.15.4. The IPsec [52] is a security protocol to provide secure E2E communications at the IP layer. Although the IPsec is mandatory with IPv6, it may not be suitable for the resource constrained devices in the 6LoWPAN networks because these devices may not have enough capability to perform all of the IPsec functions [28].

To adapt the IPsec to nodes with limited power, Granjal et al. [53] proposed two compressed security headers AH (Authentication Header) [54] and ESP (Encapsulated Security Payload) [55] to enable the E2E protection on 6LoWPAN nodes. However, only the header dispatch value of two different modes, which are transport and tunnel 
modes, and the format of the compressed header are presented while the exact the specifications of these two compressed headers are not provided.

Raza et al. [56] proposed a secure E2E communication scheme for 6LoWPAN. The work designs, implements and evaluates a compressed lightweight 6LoWPAN extension for IPsec. The detailed IPsec specification and implementation for 6LoWPAN are presented. They have extensively evaluated their implementation and demonstrated that it is possible and feasible to use compressed IPsec to secure communication between devices. Through the application of IPsec, 6LoWPAN nodes are able to authenticate, encrypt, and check the integrity of messages using standardized and established IPv6 mechanisms.

To extend the work of [56] in which only the detailed specification of compressed $\mathrm{AH}$ header is discussed, a specification for compressed ESP header was proposed in [57]. A thorough experimental evaluation of the adoption of the two proposed compressed headers along with a comparison against the link layer security of IEEE 802.15.4 are presented. This proposal is based on the LOWPAN_IPHC (IPv6 header compression) [32]. The evaluation results further demonstrate that the compressed IPsec is feasible to be employed in the 6LoWPAN to enable E2E communication. Although some existing proposals and drafts have proved that the IPsec can be compressed to protect the communication transmitted over the 6LoWPAN network, the official specifications of 6LoWPAN are still not finalized. Therefore, future research works on secure E2E communication together with the key management solutions, e.g. Internet Key Exchange (IKE), are demanded.

\subsection{Summary}

In this chapter, the state-of-the-art security solutions for the 6LoWPAN network are reviewed and classified into five major categories of detection, authentication, key management, privacy and compressed IPsec schemes. These security issues and corresponding solutions in the 6LoWPAN networks have paved the way for the further research in this area. Security mechanisms of these five categories are still needed to be enhanced. The IDSs in 6LoWPAN are required for the development because the IDS is 
a security approach to detect intrusions or attacks and discover the anomaly, which will be much beneficial to the 6LoWPANs [58]. To support massive devices, new efficient group authentication mechanisms are required for the future 6LoWPAN. The development of key management systems for the 6LoWPAN among nodes has become more important to be promoted. Further deliberated solutions of security issues in 6LoWPAN are needed to ensure the E2E secure communications at the network layer. Security header mapping mechanisms between the 6LoWPAN and the Internet are required [59].

As a summary, the existing 6LoWPAN security mechanisms are inadequate and need to be improved to make the communication environment more secure. In future, new security threats and solutions could also be discovered to cope with security and privacy issues, such as key management, secure routing, data encryption, etc. 


\section{Chapter 3 A Mutual Authentication and Key Establishment}

\section{Scheme for M2M Communication in 6LoWPAN Networks}

\subsection{Introduction}

In recent years, the M2M communication has been subjected to extensive research efforts. Unlike the traditional Internet, the devices deployed in the M2M systems could have such limited resources that the mature communication technologies cannot be applied directly. To achieve the implementation of the all-IP network, 6LoWPAN is proposed to allow IP technologies to adapt to those resources constrained devices which largely promotes the development of M2M. Though 6LoWPAN [60] is a promising technology, it has various security issues unspecified while many threats and trust crises are existing along with its development. As reviewed in the previous chapter, many research solutions on node authentication, such as [41-48], have been proposed to overcome the vulnerabilities of the 6LoWPAN systems.

As a related research area, different authentication approaches have been proposed to support security and try to meet the security requirements of the M2M communication systems. An efficient password-based authentication and key agreement scheme has been proposed in [61] to provide the security for M2M home network services. Motivated to improve the security functionalities of the M2M communications, a dynamic encryption authentication between mobile devices and the M2M service provider with low cost has been designed in [62]. The security functionalities have been significantly improved by applying the proposed lightweight encryption algorithm. However, it still depends on a pre-shared key space and the noncompromised sensor nodes.

With the rapid development of mobile communication techniques, a more effective and suitable protocol to handle fast handovers for MNs is needed. A handover authentication scheme based on the tickets for IEEE $802.16 \mathrm{~m}$ networks is proposed in [63] to perform the handover authentication between the mobile station (MS) and the 
base station (BS). Though the MS and the BS can fast authenticate with each other using the handover ticket, the pairwise master key is shared between the MS and all BSs which is insecure if one of the BS is compromised by an adversary. A ticket-based authentication protocol in [64] can also achieve fast handover authentications. However, the access point (AP) needs to generate a ticket for each neighbor AP. It will incur high storage consumption for the devices with the limited power supplies.

In this chapter, as the major contribution, an enhanced authentication and key establishment scheme is designed for M2M communications in the 6LoWPAN networks (EAKES6Lo). To overcome the shortcomings mentioned above, a hybrid cryptography approach is employed for secure authentication and flexible key establishment with the consideration of the resource constraints at the 6LoWPAN devices. A handover ticket is generated for a mobile device to achieve a fast authentication when performing handovers. Thus, the full authentication process will only be executed once the ticket is expired, which can largely improve the handover efficiency. Besides, this proposal has an outstanding feature to provide the security supports to both static and mobile devices in the 6LoWPAN networks. The security proof by the Protocol Composition Logic (PCL) can derive the logic correctness of the proposed scheme. A formal verification by using Automated Validation of Internet Security Protocols and Applications (AVISPA) has been performed to prove that the proposed scheme is able to prevent against many malicious attacks. Moreover, the simulation results by Matrix Laboratory (MATLAB) show that the proposed scheme in 6LoWPAN could incur less computational and transmission overhead.

\subsection{System Background}

\subsubsection{LoWPAN System Architecture}

The architecture of the 6LoWPAN networks under the EAKES6Lo is shown in Figure 3.1. The system consists of a number of 6LHs, some 6LRs, a 6LER and a remote server. The $6 \mathrm{LHs}$, also known as the end devices, which can be mobile or static, are used for gathering sensory information, while they do not have the routing function. 
The mobile 6LHs can move across different domains within the network, while the authentications are required when they come into a new 6LR's domain. The 6LRs are the essential components for forwarding messages between the 6LoWPAN devices. The 6LHs and 6LRs are resources constrained on both energy and computation. The 6LER, which connects the 6LoWPAN network and the IPv6 network, can gather sensory information forwarded from the 6LRs and transmit them to the remote server. The 6LER, works as a gateway, has more storage and computing capacities.

The 128-bit IPv6 address of a 6LoWPAN node contains a 64-bit network prefix and a 64-bit IID. The IID can be the global unique IEEE-defined EUI-64 or can be derived from a 16-bit short address which is unique within a PAN. If the short address is used, the 48-bit MAC ${ }^{1}$ address is formed by a 16-bit PAN ID, 16-bit zeros and the 16-bit short address. To derive the 64-bit IID from the MAC ${ }^{1}$ address, 16-bit 0xffee is inserted into the middle of the MAC ${ }^{1}$ address.

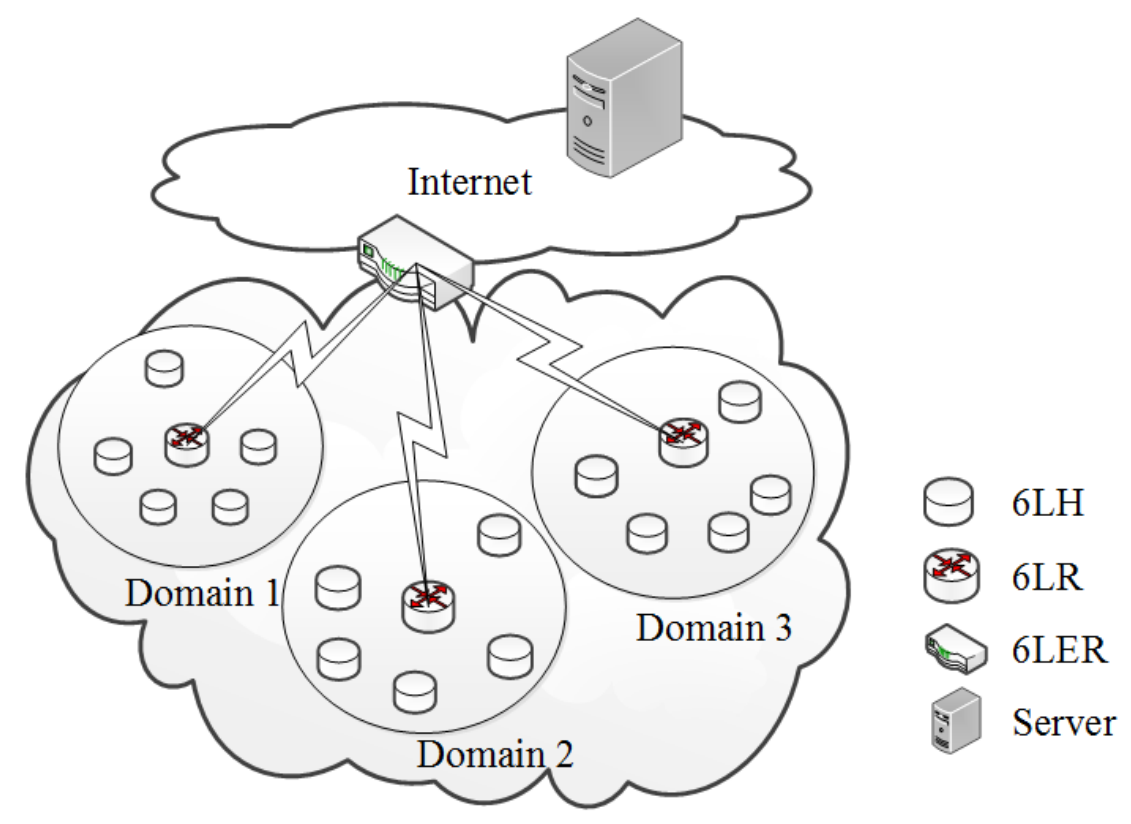

Figure 3.1 6LoWPAN System Architecture of EAKES6Lo

\subsubsection{Elliptic Curve Diffie-Hellman}

As a variant of the Diffie-Hellman scheme for the elliptic curves, Elliptic Curve Diffie-Hellman (ECDH) is utilized to set up a shared secret between the 6LH and the 
server. First, an elliptic curve $E$ over a finite field $F_{p}$, a base point $G$, and other parameters are needed to be agreed between two parties, Alice and Bob. Second, Alice and Bob generate their own private/public key pair. The private key of Alice is an integer $s_{A}$ which is randomly chosen from $\{1, \ldots, n-1\}$, where $n$ is the prime order of the subgroup. The public key is calculated by $Q_{A}=s_{A} G$ using the elliptic curve point addition. Similarly, Bob has his own private key $s_{B}$ and public key $Q_{B}=s_{B} G$. Then, Alice and Bob will exchange their public keys while keeping private keys secret. When receiving the public key of Bob, Alice will compute $s_{A} Q_{B}$. Bob will compute $s_{B} Q_{A}$ after receiving Alice's public key. The shared secret that they both calculate are the same, because $s_{A} Q_{B}=s_{A}\left(s_{B} G\right)=s_{B}\left(s_{A} G\right)=s_{B} Q_{A}$. This shared secret can be used as a symmetric key to encrypt/decrypt the exchanged messages.

\subsubsection{Attack Model}

The resource constrained 6LoWPAN devices are usually placed in unattended and accessible locations without specific supervision and more easily attacked by adversaries compared to traditional equipment which has enough resources. The threat model to the EAKES6Lo works based on the Dolev-Yao intruder model [65], which is a classic intruder model used to analyze security protocols. This model is versatile and can be used in a wide range of application scenarios. Besides, the intruder model is extended in our research by considering compromised devices. The intruder could interact with other network entities as a legitimate device by using valid credentials. Moreover, Dolev-Yao has been widely used in logic proof or automatic verification tools. The attacker may be an outsider or a legitimate principal in the system that can:

- $\quad$ Eavesdrop on any message transmitted through the network.

- $\quad$ Alter, decompose, synthesize, forge, replay or inject any message and send it to a legitimate entity.

- Impersonate a legal principal by using valid credentials.

- Decrypt or encrypt messages if obtaining the corresponding secret key.

However, it is assumed that there are certain things that an adversary is unable to: 
- Guess a nonce which is randomly generated from a sufficiently large space.

- Retrieve the information from a given ciphertext or generate a valid ciphertext from a given plaintext without a complete and correct key.

- Calculate the corresponding private key given a public key.

\subsection{Review of the SAKE Scheme}

\subsubsection{Details of the SAKES Scheme}

The SAKES scheme [42] is the first state-of-the-art solid solution to provide authentication and key establishment for 6LoWPAN networks. Therefore, the details of the operations of the SAKES will be reviewed in this section. It is assumed that all the hosts and routers, which have registered to the 6LER, are static and the 6LER and the 6LRs have more sufficient computation and storage capabilities. The notations used are listed in Table 3.1.

Table 3.1 Notations Used in Chapter 3

\begin{tabular}{cc}
\hline \hline Notation & Description \\
\hline$I D_{x}$ & Identity of $x$ \\
$N_{x}$ & Nounce generated by $x$ \\
$T_{x}$ & Timestamp generated by $x$ \\
$T_{E X P}$ & Expiration timestamp generated by the server \\
$K_{x \leftrightarrow y}$ & Symmetric key shared between $x$ and $y$ \\
$K p r i_{x}$ & Private Key of $x$ \\
$K p u b_{x}$ & Public Key of $x$ \\
$H K$ & Secret key used for generating the MAC \\
$S K_{x \leftrightarrow y}$ & Session key established between $x$ and $y$ \\
\hline \hline
\end{tabular}




\section{1) Authentication Phase}

A 6LH first broadcasts a HELLO request searching for neighbor 6LRs. When the 6LH receives the HELLO reply from a neighbor $6 \mathrm{LR}$, the $6 \mathrm{LH}$ will compute $\mathrm{Ci}=$ $\left(I D_{6 L H}, I D_{6 L R}, I D_{S E R}, N_{6 L H}\right) K_{6 L H \leftrightarrow 6 L E R}$ and the message authentication code (MAC $\left.{ }^{2}\right)$ $M A C=\operatorname{hash}\left(C i, K_{6 L H \leftrightarrow 6 L E R}, I D_{6 L H}, N_{6 L H}\right)$, and send the request message < $M A C, C i, I D_{6 L H}, N_{6 L H}>$ to the neighbor 6LR for forwarding the message.

Once the $6 \mathrm{LR}$ gets the message, it will check the MAC ${ }^{2}$ of the received message. After verifying the $\mathrm{MAC}^{2}$, the $6 \mathrm{LR}$ will compute $M A C=\operatorname{hash}\left(C i, K_{6 L R \leftrightarrow 6 L E R}\right.$, $\left.I D_{6 L R}, N_{6 L R}\right)$ and send the message $<M A C, C i, I D_{6 L R}, N_{6 L R}>$ to the 6LER.

When the 6LER receives the message, the 6LER will check whether the 6LH and the $6 \mathrm{LR}$ have been registered with the $6 \mathrm{LER}$ and the $\mathrm{MAC}^{2}$ is valid. After verifying the message and the identities, the 6LER will send the message $<\left(N_{6 L E R},\left(I D_{6 L H}, I D_{6 L R}\right.\right.$, $\left.\left.I D_{S E R}\right) \operatorname{Kpri}_{6 L E R}, K p u b_{6 L R}, \operatorname{Kpri}_{6 L R}\right) K_{6 L R \leftrightarrow 6 L E R}>$ which contains a lightweight public-private key combination based on the Elliptic Curve Cryptography (ECC) to the $6 \mathrm{LR}$ and the message $<\left(I D_{6 L R}, N_{6 L E R}\right) K_{6 L H \leftrightarrow 6 L E R}>$ to the $6 \mathrm{LH}$, respectively. If the $6 \mathrm{LR}$ is not registered with the 6LER, the 6LER will broadcast the identity of the suspected 6LR to other devices in the 6LoWPAN and look for an authenticated 6LR nearest to the $6 \mathrm{LH}$ from the registry of the 6LER. It then decides the correct path to reach the request.

2) Key Establishment Phase

The $6 \mathrm{LR}$ sends a request message $<\left(M A C, C i, I D_{6 L R}, N_{6 L R}\right) K p r i_{6 L R}>$ to the server where $C i=\left(I D_{6 L H}, I D_{6 L R}, I D_{S E R}\right) K_{p r i} i_{6 L E R}$ and $M A C=\operatorname{hash}\left(C i, I D_{6 L R}\right.$, $\left.N_{6 L R}, K p u b_{6 L R}\right)$. The server will check the $\mathrm{MAC}^{2}$ of the received message. If it is valid, the server will calculate the session key $S K_{S E R \leftrightarrow 6 L H}=g^{\text {Kpub }_{6 L R} * K_{\text {Kri }}}{ }_{S E R} \bmod p$ by using Diffie-Hellman key exchange method. Then, the server will send the reply message $<\left(M A C, I D_{S E R}, p, g, N_{S E R}\right) K p r i_{S E R}>$ to the $6 \mathrm{LR}$, where $M A C=$ $\operatorname{hash}\left(I D_{S E R}, K p u b_{S E R}, p, g, N_{S E R}\right)$.

Since the $6 \mathrm{LH}$ is resource constrained, the $6 \mathrm{LR}$ will calculate the session key

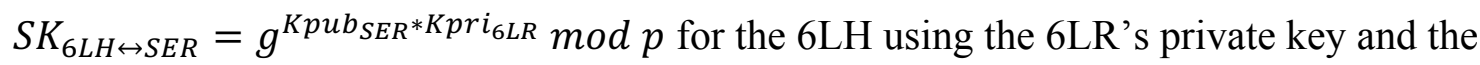


server's public key. After that, the session key $S K_{6 L H \leftrightarrow S E R}$ will be sent to the $6 \mathrm{LH}$, so the $6 \mathrm{LH}$ can communicate with the remote server in a secure way.

\subsubsection{Vulnerabilities of the SAKES Scheme}

We have investigated the details of the operation of the SAKES carefully with the exploration of various vulnerabilities of the SAKES scheme. The major shortcomings of the SAKES scheme can be found as follows.

1) Vulnerability to Compromised Attacks

The security analysis on the SAKES states that it has the ability to protect against sinkhole and wormhole attacks. However, these attacks can only be prevented under the assumption that the 6LHs and the 6LRs have not been compromised. By the SAKES scheme, the session key between the 6LH and the server is generated by the 6LR, which means that the 6LR has all the session keys of the 6LHs within its domain and can decrypt the messages sent by the 6LH or the server. Since the session key established between the 6LH and the server will be known to an attacker if a 6LR is compromised, the information forwarded by the 6LR will all be exposed. Moreover, the routers' private/public key pairs are generated by the 6LER which is energy consuming for a resource constrained 6LoWPAN device. If the 6LER is compromised, all the keys can be obtained and the session keys established between the 6LH and the remote server can be easily computed.

2) Lack of Mobility Support

By the SAKES, the configuration assumption is that all devices are static. However, in the real-world applications, the 6LHs or even the 6LRs could be mobile. For example, when a patient is moving within a hospital with several sensors, it is important to provide mobility support for those sensors so that the real-time monitoring can be continued without an interruption. With the rapid development of wireless technologies and the increasing demand for more flexible and convenient mobile devices, the support for MNs is required in 6LoWPAN systems. Although the SAKES scheme could adapt to the mobility of the MNs, each re-authentication costs too much computational overhead when a handover is performed. 


\subsection{Proposed Scheme}

\subsubsection{Motivation}

In order to overcome the shortcomings of SAKES, the enhanced authentication and key establishment (AKE) scheme is designed with novel ideas. To improve the ability to defend against compromised attacks, the 6LR is able to include more information to the messages sent from the 6LH or the 6LER with a limited ability for the decryption. Besides, to reduce the risk when the 6LR is compromised, the 6LH should generate the private/public key pair itself. Moreover, in order to support the mobile applications, mobile hosts should be included in the system architecture. The mobile 6LHs need to be authenticated when they come into a new 6LR's domain.

\subsubsection{Details of the Proposed EAKES6Lo}

To enhance the security functionality of the 6LoWPAN networks, the EAKES6Lo scheme is designed in the 6LoWPAN networks for the secure M2M communications. It consists of three phases: 1) pre-deployment phase, 2) AKE phase and 3) handover phase. The 6LHs, whether it is static or mobile, need to perform the first two steps to ensure their legitimacy and prevent unauthorized disclosure of information. The MNs need to follow the third step when performing handovers. The notations used in EAKES6Lo are listed in Table 3.1. It is assumed that all the devices have a unique 64bit IID or 16-bit short address within a single LoWPAN and the link between the 6LER and the remote server is secure.

1) Pre-deployment Phase

Before the deployment of a new $6 \mathrm{LH}$, a parameter list $\{p, q, a, b, G, n, h\}$ of ECC is published by the system through the following steps:

- Choose a $k$-bit prime number $p$ specifying the finite field $F_{p}$.

- Choose two coefficients $a$ and $b$ specifying an elliptic curve $E / F_{p}$ which is defined by the equation $y^{2}=x^{3}+a x+b \bmod p$. 
- $\quad$ Choose a base point $G=(x, y)$ on $E\left(F_{p}\right)$ that generates the subgroup whose prime order is $n$ and cofactor is $h$

After publishing the parameter list, a public/private key pair can be generated based on these parameters. For example, a secret number $r_{i} \in Z_{q}^{*}$ ( $q$ is a prime number) is selected to be the private key of a $6 \mathrm{LH} i$ and $R_{i}\left(=r_{i} G\right)$ is its public key.

By the EAKES6Lo, prior to the deployment of a new host $i$, each host in the 6LoWPAN network has a unique identity such as the MAC ${ }^{1}$ address and must be registered with the server. The host $i$ sends a registration request to the server with its ID and its public key. After checking that the ID has not been registered before, the server will send its public key and a randomly generated number $S_{i}$ in response to the request and store $I D_{i}, K p u b_{i}$ and $S_{i}$ in the database which will be further used to produce the symmetric key between the host $i$ and the remote server. The registration steps are depicted in Figure 3.2.

$\begin{array}{lll}\text { Host } \boldsymbol{i} & \text { Registration Req. }\left\langle I D_{i} \| K p u b_{i}\right\rangle \\ \longleftarrow & \text { Registration Res. }\left\langle S_{i} \| K p u b_{S}\right\rangle\end{array}$

Figure 3.2 Registration Phase of EAKES6Lo

The symmetric key $K_{H_{i} \leftrightarrow S E R}$ between the $6 \mathrm{LH} i$ and the remote server is calculated by both parties in advance in order to save more time when performing the AKE phase. The key $K_{H_{i} \leftrightarrow S E R}$ is computed by itself using ECDH and simple hash function through the function $K_{H_{i} \leftrightarrow S E R}=\operatorname{Hash}\left(\operatorname{Kpri}_{H_{i}} \times K p u b_{S E R}, S_{i}\right)$, while key $K_{S E R \leftrightarrow H_{i}}$ is obtained by the server calculating $K_{S E R \leftrightarrow H_{i}}=\operatorname{Hash}\left(\operatorname{Kpri}_{S E R} \times K p u b_{H_{i}}, S_{i}\right)=K_{H_{i} \leftrightarrow S E R}$.

2) Authentication and Key Establishment Phase

In this phase, the 6LHs, the 6LRs, the 6LER and the server will exchange six messages to achieve a mutual authentication as shown in Figure 3.3. The details of the AKE process are described as follows. 


$$
\begin{aligned}
& \begin{array}{llll}
\text { 6LH } & \text { 6LR } & \text { 6LER } & \text { Server }
\end{array} \\
& \left\{I D_{6 L H}\left|I D_{6 L R}\right| T_{6 L H} \mid\left\{N_{6 L H}\right\} K_{6 L H \leftrightarrow S E R}\right\} K \operatorname{Pri}_{6 L H} \\
& \left\{I D_{6 L H}\left|I D_{6 L R}\right| T_{6 L H} \mid\left\{N_{6 L H}\right\} K_{6 L H \leftrightarrow S E R}\right\} K_{\text {pri }} i_{6 L H} \\
& \left\{I D_{6 L H}\left|I D_{6 L R}\right| T_{6 L H} \mid\left\{N_{6 L H}\right\} K_{6 L H \leftrightarrow S E R}\right\} K p r i_{6 L H} \mid\left\{T_{6 L E R} \mid M A C 1\right\} K p r i i_{6 L E R} \\
& M A C 1=H_{M A C}\left(T_{6 L E R}\left|I D_{6 L H}\right| I D_{6 L R}\left|I D_{6 L E R}\right| K p u b_{6 L H}\left|K p u b_{6 L R}\right| K p u b_{6 L E R}\right) \\
& \left\{I D_{6 L H}\left|I P v 6_{6 L H}\right| K p u b_{6 L H}\left|T_{S E R}\right| T_{E X P}\right\} \operatorname{Kpri}_{S E R} \mid M A C 2 \\
& M A C 2=H M A C_{H K}\left(\text { Ticket } \mid N_{6 L H}\right) \\
& \left\{I D_{6 L H}\left|I P v 6_{6 L H}\right| K_{p u b} b_{6 L H}\left|T_{S E R}\right| T_{E X P}\right\} K \operatorname{Pri}_{S E R} \mid M A C 2 \\
& M A C 2=H M A C_{H K}\left(\text { Ticket } \mid N_{6 L H}\right) \\
& \left\{I D_{6 L H}\left|I P v 6_{6 L H}\right| K p u b_{6 L H}\left|T_{S E R}\right| T_{E X P}\right\} K \operatorname{Pri}_{S E R} \mid M A C 2 \\
& M A C 2=H M A C_{H K}\left(\text { Ticket } \mid N_{6 L H}\right)
\end{aligned}
$$

Figure 3.3 Authentication and Key Establishment Phase of EAKES6Lo

When a new 6LH wants to join in a LoWPAN, it will perform neighbor discovery defined in [66] to obtain its unique global IPv6 address. A nonce $N_{6 L H}$ is randomly chosen by the $6 \mathrm{LH}$ to be encrypted by symmetric key $K_{6 L H \leftrightarrow S E R}$. Then, $I D_{6 L H}, I D_{6 L R}$ received through router advertisement, timestamp $T_{6 L H}$ generated by the $6 \mathrm{LH}$, encrypted nonce and the signature will be sent out to the 6LR as message 1 by the $6 \mathrm{LH}$. The content of message 1 is as follows.

$$
\left\{I D_{6 L H}\left|I D_{6 L R}\right| T_{6 L H} \mid\left\{N_{6 L H}\right\} \mathrm{K}_{6 L H \leftrightarrow S E R}\right\} K K \operatorname{ri}_{6 L H}
$$

The 6LR, which gets message 1 from the $6 \mathrm{LH}$, will verify the signature. If the signature is valid and the identity of the router contained in the message is correct, message 1 is going to be forwarded to the 6LER as message 2.

When the 6LER receives the message from the $6 \mathrm{LR}$, it will verify the signature of this message. If it is valid, the 6LER will compare the identities $I D_{6 L R}$ and $I D_{6 L H}$ with the registered ID list and check whether the identities and the addresses of the devices are consistent. If the 6LR has not been registered or the message forwarded by the 6LR and the information related to the router contained in the message are not the same, the 
6LER will inform other 6LRs and 6LHs, and add the suspected 6LR to the black list. When the $6 \mathrm{LH}$ gets the response message from the 6LER, it will find another 6LR to transmit the information. If the 6LR is a legitimate device, the message forwarded by the 6LR, a timestamp $T_{6 L E R}$ and the value $M A C 1$ will be signed by the 6LER and sent out to the server as message 3 to confirm that the host has access to the 6LoWPAN network. The content of message 3 is as follows.

$$
\begin{aligned}
& \left\{I D_{6 L H}\left|I D_{6 L R}\right| T_{6 L H} \mid\left\{N_{6 L H}\right\} K_{6 L H \leftrightarrow S E R}\right\} K_{\text {pri }} i_{6 L H} \mid\left\{T_{6 L E R} \mid M A C 1\right\} \text { Kpri }_{6 L E R} \\
& M A C 1=\operatorname{Hash}_{H K}\left(T_{6 L E R}\left|I D_{6 L H}\right| I D_{6 L R}\left|I D_{6 L E R}\right| K p u b_{6 L H}\left|K p u b_{6 L R}\right| K p u b_{6 L E R}\right)
\end{aligned}
$$

After receiving the message 3, the server will first check if the time of transmission is below the defined threshold using the two timestamps included in the message and the receiving time. Then, the information relevant to the $6 \mathrm{LH}$ will be retrieved from the list of registered devices. Next, the signature signed by the 6LH and the 6LER will be verified using the corresponding public key stored in server's database. The MAC ${ }^{2}$ value of $T_{6 L E R}, I D_{6 L H}, I D_{6 L R}, I D_{6 L E R}, K p u b_{6 L H}, K p u b_{6 L R}$ and $K p u b_{6 L E R}$ is compared with the received one to ensure the integrity of the received data. If the $6 \mathrm{LH}$ has not been registered, a warning message will be sent to the 6LER and will be broadcasted to the whole 6LoWPAN network that the 6LH is an illegitimate device. If the 6LH is confirmed to be legitimate, the nonce $N_{6 L H}$ can be derived from the encrypted message by symmetric key $K_{S E R \leftrightarrow 6 L H}$. A ticket, which is employed during the handover phase, is calculated by the function Ticket $=\left\{I D_{6 L H}, I P v 6_{6 L H}, K p u b_{6 L H}, T_{S E R}, T_{E X P}\right\} K p r i_{S E R}$. The ticket and a new $\mathrm{MAC}^{2}$ value computed by the ticket and the nonce $N_{6 L H}$ are sent to the 6LER as message 4 as follows.

$$
\begin{aligned}
& \left\{I D_{6 L H}\left|I P v 6_{6 L H}\right| K_{\text {pub }} b_{6 L H}\left|T_{S E R}\right| T_{E X P}\right\} \text { Kpri }_{S E R} \mid M A C 2 \\
& \left.M A C 2=\text { Hash }_{H K} \text { (Ticket } \mid N_{6 L H}\right)
\end{aligned}
$$

When the 6LER receives the message sent by the server, it verifies the signature, stores the $I D_{6 L H}$ in the list of the legitimate devices and forwards the message to the 6LR as message 5. Similarly, the 6LR will perform the same procedure as the 6LER and send the message as message 6 to the $6 \mathrm{LH}$.

After receiving the forwarded message from the $6 \mathrm{LR}$, the $6 \mathrm{LH}$ checks whether the $\mathrm{MAC}^{2}$ value is valid and stores the ticket that can be further used when a handover 
occurs. Finally, the new 6LH has authenticated with the remote server and a secure connection between them has been set up.

3) Handover Phase

When a mobile $6 \mathrm{LH}$ is going to perform a handover, the handover ticket issued during the authentication phase will be used to achieve a fast authentication with the 6LRs. When a mobile 6LH moves from the area of 6LR1 to the area of 6LR2, it will send a handover request to $6 \mathrm{LR} 2$, which contains the handover ticket, a timestamp and the $M A C 3$. The handover steps are depicted in Figure 3.4.

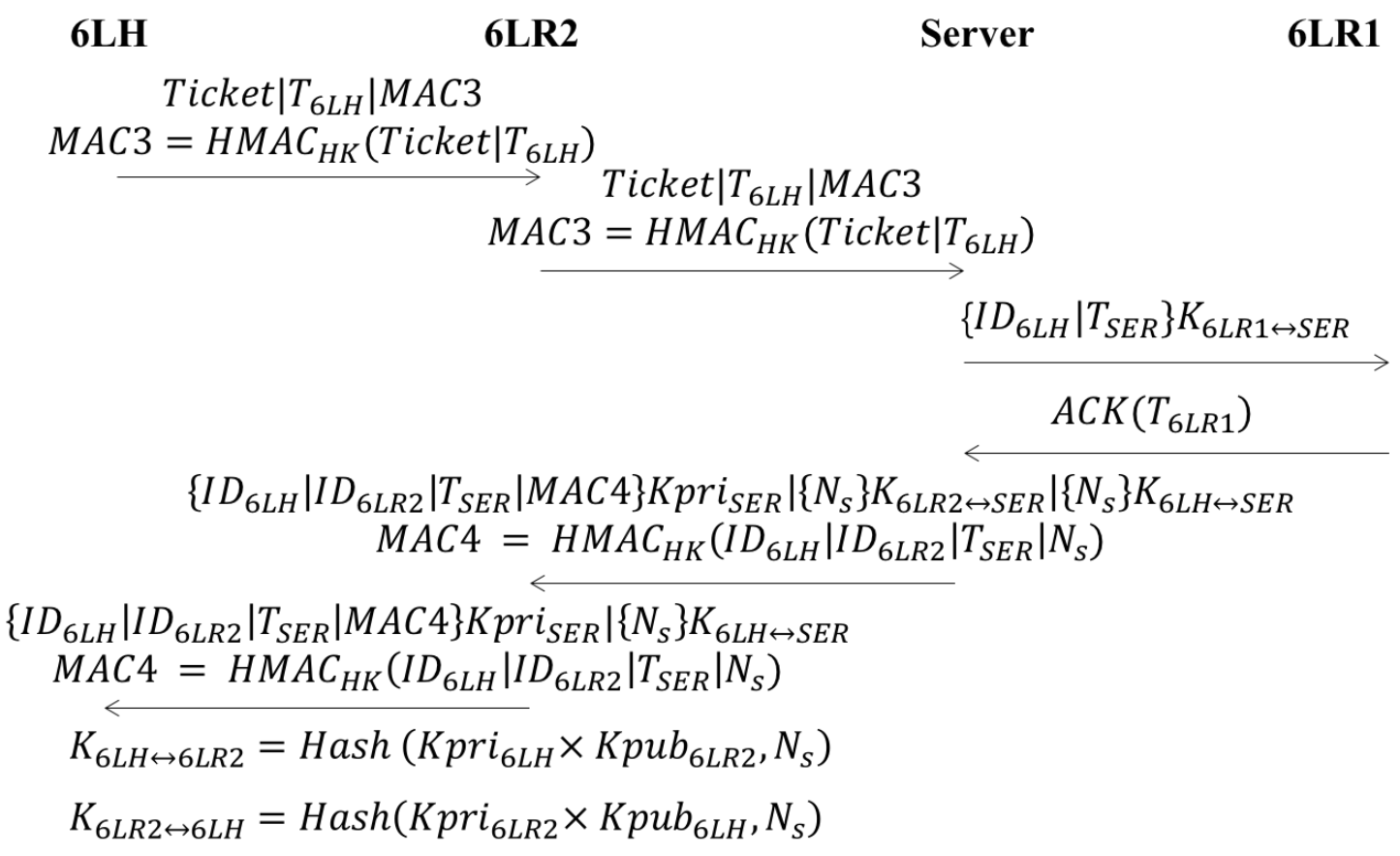

Figure 3.4 Handover Phase of EAKES6Lo

Ticket $\left|T_{6 L H}\right| M A C 3$

$M A C 3=\operatorname{Hash}_{H K}\left(\right.$ Ticket $\left.\mid T_{6 L H}\right)$

Upon receiving the request from the 6LH, the 6LR2 should make sure whether the ticket is expired. If so, the request is discarded. Otherwise, the 6LR2 verifies the signature and the value of $M A C 3$. If they are valid, a message will be sent to the server for authentication. When the server receives the message, it will verify the authenticity 
of $6 \mathrm{LH}$ and 6LR2. Then, a notification will be sent to the 6LR1 to inform that the 6LH is no longer in its area. The 6LR1 checks if the $6 \mathrm{LH}$ is out of its range and deletes the device from its member list. An acknowledgement (ACK) will be sent back to the server. When receiving the confirmation from the $6 \mathrm{LR} 1$, a random number will be generated by the server for establishing a symmetric key shared between the $6 \mathrm{LH}$ and the 6LR2. The content of the message sent to the 6LR2 is as follows.

$$
\begin{aligned}
& \left\{I D_{6 L H}\left|I D_{6 L R 2}\right| T_{S E R} \mid M A C 4\right\} K p r i \_S E R\left|\left\{N_{S}\right\} K_{6 L R 2 \leftrightarrow S E R}\right|\left\{N_{S}\right\} K_{6 L H \leftrightarrow S E R} \\
& M A C 4=\operatorname{Hash}_{H K}\left(I D_{6 L H}\left|I D_{6 L R 2}\right| T_{S E R} \mid N_{S}\right)
\end{aligned}
$$

After receiving the message, the random number will be stored by the 6LR2 if the signature, timestamp and the $\mathrm{MAC}^{2}$ value are valid and the rest of the message will be transmitted to the $6 \mathrm{LH}$. Similarly, the $6 \mathrm{LH}$ verifies the validity of the message and stores the nonce $N_{s}$. Then the symmetric key shared between the $6 \mathrm{LH}$ and the 6LR2 can be calculated as follows.

$$
\begin{aligned}
& K_{6 L H \leftrightarrow 6 L R 2}=\operatorname{Hash}\left(\operatorname{Kpri}_{6 L H} \times K p u b_{6 L R 2}, N_{S}\right) \\
& K_{6 L R 2 \leftrightarrow 6 L H}=\operatorname{Hash}\left(\operatorname{Kpri}_{6 L R 2} \times K p u b_{6 L H}, N_{S}\right)
\end{aligned}
$$

\subsection{Security Analysis}

In this section, the proposed EAKES6Lo scheme is analyzed from three aspects. First, a proof of the logic correctness by using PCL will be provided to model the EAKES6Lo scheme and derive its corresponding security properties. Then, a formal verification is taken by using AVISPA to automatically verify the security functionality. Finally, further analysis and explanation on the ability against various malicious attacks of the proposed solution are provided.

\subsubsection{Security Proof}

PCL [67, 68] is a formal logic to prove the logic correctness and find vulnerabilities of a given protocol. The sequence of the actions is proved by PCL with the consideration of the given sequence of actions and arbitrary actions of attackers. PCL can be used to prove security properties of network protocols without reasoning about an attacker while only reasoning about the actions of honest roles under unknown 
environmental conditions. By PCL, there are three steps of proving the logic correctness of a given protocol using PCL: 1) Model the protocol: The actions of each participants will be written in a protocol programming language. 2) Specify the preconditions and invariants: Initial assumptions and invariants are specified, such as which two entities have a shared secret. 3) Prove the security property: The conclusion on whether the security property that each sending and receiving event happen in order has been achieved or not will be derived using PCL axioms and rules. The notations used in PCL are listed in Table 3.2.

Table 3.2 Notations Used in PCL

\begin{tabular}{|c|c|c|}
\hline & Notations & Descriptions \\
\hline \multirow{7}{*}{ Terms } & $\hat{X}, \hat{Y}, \hat{Z}, \hat{S}$ & Principals \\
\hline & $X, Y, Z, S$ & Threads \\
\hline & $x$ & Basic term variable \\
\hline & $u$ & Basic term \\
\hline & $K$ & Key \\
\hline & $E N C_{K}\{|x|\}$ & Term encrypted with key $K$ \\
\hline & $S I G_{K}\{|x|\}$ & Term signed with key $K$ \\
\hline \multirow{8}{*}{ Actions } & Send $u$ & Send a term $u$ \\
\hline & Receive $x$ & Receive term into variable $x$ \\
\hline & New $x$ & Generate new term $x$ \\
\hline & Match $u / u$ & Match a term to a pattern \\
\hline & $x:=\operatorname{sign} u, K$ & Sign the term $u$ using key $K$ \\
\hline & Verify $x, u, K$ & Verify the signature \\
\hline & $x:=$ symenc $u, K$ & Encrypt the term $u$ using symmetric key $K$ \\
\hline & $x:=$ symdec $u, K$ & Decrypt the term $u$ using symmetric key $K$ \\
\hline
\end{tabular}


Before modelling the EAKES6Lo by PCL, the parties involved in the operation of the protocol and the messages exchanged among them should be explicitly described in a simplified arrows-and-messages way as follows:

$6 \mathrm{LH} \rightarrow 6 \mathrm{LR}:\left(I D_{6 L H}, I D_{6 L R}, T_{6 L H},\left(N_{6 L H}\right) K_{6 L H \leftrightarrow S E R}\right) K p r i_{6 L H}$

6LR $\rightarrow$ 6LER: $\left(I D_{6 L H}, I D_{6 L} R, T_{6 L H},\left(N_{6 L H}\right) K_{6 L H \leftrightarrow S E R}\right) K p r i_{6 L H}$

6LER $\rightarrow$ Server: $\left(T_{6 L E R}, M A C 1\right) K p r i_{6 L E R},\left(\begin{array}{c}I D_{6 L H}, I D_{6 L R}, T_{6 L H} \\ \left(N_{6 L H}\right) K_{6 L H \leftrightarrow S E R}\end{array}\right) K p r i_{6 L H}$

Server $\rightarrow 6$ LER: $M A C 2$, ticket

$$
\left(\text { ticket }=\left(I D_{6 L H}, I P v 6_{6 L H}, K p u b_{6 L H}, T_{S E R}, T_{E X P}\right) \text { Kpri }_{6 L H}\right)
$$

6LER $\rightarrow 6 \mathrm{LR}: M A C 2$, ticket

6LR $\rightarrow 6 \mathrm{LH}:$ MAC2, ticket

1) Protocol Modelling

By the PCL, the first step of the security proof is the protocol modelling, which is to express the execution steps of all the participants, also called principals, in detail to formally prove the security properties of a protocol [67]. By the EAKES6Lo, there are four participants including $6 \mathrm{LH}, 6 \mathrm{LR}, 6 \mathrm{LER}$ and server which are described as $\hat{X}, \hat{Y}, \hat{Z}$ and $\hat{S}$ respectively.

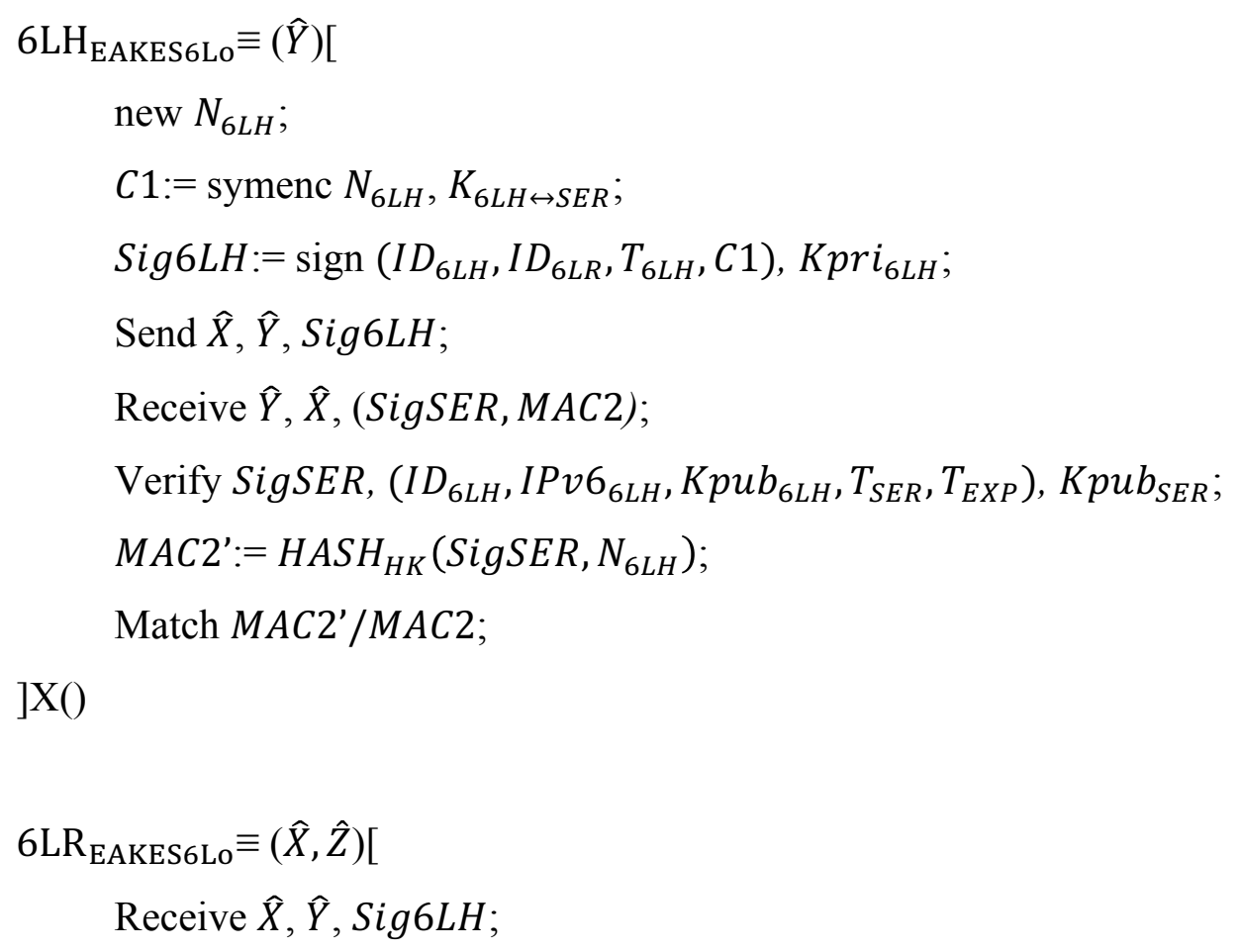


Verify Sig6LH, $\left(I D_{6 L H}, I D_{6 L R}, T_{6 L H}, C 1\right), K p u b_{6 L H}$;

Send $\hat{Y}, \hat{Z}, \operatorname{Sig} 6 L H$;

Receive $\hat{Z}, \widehat{Y},($ SigSER, MAC2);

Verify SigSER, $\left(I D_{6 L H}, I P v 6_{6 L H}, K p u b_{6 L H}, T_{S E R}, T_{E X P}\right), K p u b_{S E R}$;

Send $\hat{Y}, \hat{X},(\operatorname{SigSER}, M A C 2)$;

$\mathrm{Y}()$

$6 \operatorname{LER}_{\text {EAKES } 6 \mathrm{Lo}} \equiv(\hat{Y}, \hat{S})[$

Receive $\hat{Y}, \hat{Z}$, Sig $6 L H$

Verify Sig6LH, $\left(I D_{6 L H}, I D_{6 L R}, T_{6 L H}, C 1\right), K p u b_{6 L H}$;

$M A C 1:=H_{A S H} H_{H K}\left(T_{6 L E R}, I D_{6 L H}, I D_{6 L R}, I D_{6 L E R}, K p u b_{6 L H}, K p u b_{6 L R}\right.$, $\left.K p u b_{6 L E R}\right)$;

$\operatorname{Sig} 6 L E R:=\operatorname{sign}\left(T_{6 L E R}, M A C 1\right), K_{\text {pri }} i_{6 L E R} ;$

Send $\hat{Z}, \hat{S}$, (Sig6LH, Sig6LER);

Receive $\hat{S}, \hat{Z},($ SigSER, MAC2);

Verify SigSER, $\left(I D_{6 L H}, I P v 6_{6 L H}, K p u b_{6 L H}, T_{S E R}, T_{E X P}\right), K p u b_{S E R}$;

Send $\hat{Z}, \hat{Y},($ SigSER, MAC2);

$\mathrm{ZZ}()$

Server $_{\text {EAKES6Lo }} \equiv(\hat{Z})[$

Receive $\hat{Z}, \hat{S},($ Sig $6 L H$, Sig $6 L E R)$;

Verify Sig6LH, $\left(I D_{6 L H}, I D_{6 L R}, T_{6 L H}, C 1\right), K p u b_{6 L H}$;

Verify Sig6LER, $\left(T_{6 L E R}, M A C 1\right), K u b_{6 L E R}$;

$M A C 1:=H_{A S H} H_{H K}\left(T_{6 L E R}, I D_{6 L H}, I D_{6 L R}, I D_{6 L E R}, K p u b_{6 L H}, K p u b_{6 L R}\right.$, $\left.K p u b_{6 L E R}\right)$;

Match $M A C 1^{\prime} / M A C 1$;

$N_{6 L H}:=\operatorname{symdec} C 1, K_{6 L H \leftrightarrow S E R} ;$

SigSER: $=\operatorname{sign}\left(I D_{6 L H}, I P v 6_{6 L H}, K p u b_{6 L H}, T_{S E R}, T_{E X P}\right), K p r i_{S E R}$

$M A C 2:=\operatorname{HASH}_{H K}\left(\operatorname{SigSER}, N_{6 L H}\right)$; 
Send $\hat{S}, \hat{Z},($ SigSER, MAC2);

$\mathrm{S}()$

2) Precondition and Invariants

By the PCL, in the second stage of the security proof, the preconditions of a protocol should be stated and established. With the satisfaction of the preconditions, the protocol can be ensured to execute correctly. For example, for initiator $\hat{X}$, the precondition of the EAKES6Lo is represented as $\theta_{\text {EAKES6Lo }}$ that the 6LH and the server have the shared secret key $K_{6 L H \leftrightarrow S E R}$ before the authentication is stated.

$$
\theta_{\text {EAKES6Lo }}=(\operatorname{Honest}(\hat{X}) \wedge \operatorname{Honest}(\hat{Z})) \supset\left(\operatorname{Has}\left(\hat{X}, K_{6 L H \leftrightarrow S E R}\right) \wedge \operatorname{Has}\left(\hat{Z}, K_{6 L H \leftrightarrow S E R}\right)\right)
$$

The invariants, which describe the environment where the protocol can operate and preserve its security properties, should be stated to proceed with the security proof. By the PCL, the invariants can be proved by the honesty rule that if a property holds before each role starts, and is preserved after any sequence of actions performed by an honest principal, then the property holds for every honest principal [67]. Invariant $\Gamma_{E A K E S 6 L O}$ is described as follows.

$$
\begin{aligned}
\Gamma_{E A K E S 6 L O} & =\operatorname{Send}(Y, t 1) \wedge \text { Contains }\left(t 1, H_{A S H_{H K}}\left(\operatorname{SigSER}, N_{6 L H}\right)\right) \\
& \supset\left(\operatorname { N e w } ( Y , N _ { 6 L H } ) \vee \left(\operatorname{Receive}(Y, t 2) \wedge \text { Contains }\left(t 2, N_{6 L H}\right)\right.\right. \\
& <\operatorname{Send}(Y,(\widehat{Y}, \hat{X},(\operatorname{SigSER}, M A C 2)))))
\end{aligned}
$$

3) Security Property

By the PCL, in the final stage of the security proof, the security properties of the protocol should be demonstrated that each sending action takes place before the corresponding receiving action. Each message sent by the initiator should be received by the responder and vice versa. Moreover, the order of sending or receiving actions executed by each principal should be the same.

To prove the security property of the proposed protocol, some axioms and rules $[67,68]$ have been used summarized as follows.

AA1. $T[a]_{\mathrm{X}}$ a

AA4. $\mathrm{T}[a ; \ldots ; b]_{X} \mathrm{a}<\mathrm{b}$ 
Axiom AA1 states that if the precondition is true, after an action has been executed by a principal, the corresponding predicate which asserts that the action happened previously is true. AA4 states that after actions $a, \cdots, b$ have been done by the thread $X$ sequentially, the action predicates that the action a happened before the action $\mathrm{b}$.

AN1. $\operatorname{New}(X, x) \wedge \operatorname{New}(Y, x) \supset X=Y$

AN3. $T[\text { new } x]_{X} \operatorname{Fresh}(X, x)$

Axiom AN1 states that a nonce is created by a unique thread and AN3 says that if a nonce $x$ is generated by thread $X$, then $x$ is fresh.

REC. $\operatorname{Receive}(X, x) \supset \operatorname{Has}(X, x)$

PROJ. $\operatorname{Has}(X,(x, y)) \supset \operatorname{Has}(X, x) \wedge \operatorname{Has}(X, y)$

DEC. $\operatorname{Has}\left(X, E N C_{K}\{|x|\}\right) \wedge \operatorname{Has}(X, K) \supset \operatorname{Has}(X, x)$

REC states that a principal has the term $x$ if the principal has received it. PROJ allows a tuple to decompose itself into its components and DEC means that if the key is known, an encrypted term can be decrypted.

SEQ. $\frac{\phi_{1}[P]_{X} \phi_{2} \phi_{2}\left[P^{\prime}\right]_{X} \phi_{3}}{\phi_{1}\left[P P^{\prime}\right]_{X} \phi_{3}}$

Sequencing rule states that when the post-condition of $P$ and the pre-condition of $P^{\prime}$ matches, the two cords $P$ and $P^{\prime}$ can be sequentially composed.

P1. Persist $(X, t)[a]_{X}$ Persist $(X, t)$ for Persist $\in\{$ Has, FirstSend, $a$, Gen $\}$

Axiom P1 states that if certain predicates are true, they will be held after further actions.

FS1. Fresh $(X, t)\left[\operatorname{send} t^{\prime}\right]_{X} \operatorname{FirstSend}\left(X, t, t^{\prime}\right)$, where $t \subseteq t^{\prime}$

FS2. FirstSend $\left(X, t, t^{\prime}\right) \wedge \mathrm{a}\left(Y, t^{\prime \prime}\right) \supset \operatorname{Send}\left(X, t^{\prime}\right)<\mathrm{a}\left(Y, t^{\prime \prime}\right)$, where $X \neq Y$ and $t \subseteq t^{\prime \prime}$

Axioms FS1 states that if a thread $X$ generates a fresh nonce $t$, this nonce will be first sent in a message $t^{\prime}$ after the execution of the action sending $t^{\prime}$ by the thread $X$. Axiom FS2 says that if a term $t^{\prime \prime}$, which contains a term $t$, is involved in some actions done by a thread $Y$ and the term $t$ have been sent inside the term $t^{\prime}$ by a thread $X$ before, then the sending action by $X$ must have happened prior to $Y$ 's action.

HON. $\frac{\operatorname{Start}(X)[]_{X} \phi \quad \forall \rho \in Q . \forall P \in B S(\rho) \cdot \phi[P]_{X} \phi}{\operatorname{Honest}(X) \supset \phi}$ 
The honesty rule $\mathrm{HON}$ is applied for proving that certain actions of other parties in the protocol have been performed or not.

HASH4. Has $\left(X, \operatorname{HASH}_{K}(x)\right) \supset \operatorname{Computes}\left(Y, H_{A S H_{K}}(x)\right) \vee \exists Y, m$. Computes( $\left.Y, \operatorname{HASH}_{K}(x)\right) \wedge \operatorname{Send}(Y, m) \wedge \operatorname{Contains}\left(m, \operatorname{HASH}_{K}(x)\right)$

HASH4 states that if thread $X$ has the hash value, the hash value is computed by $X$ itself or others.

- Security Property of the $6 \mathrm{LH}$

The formal property of $6 \mathrm{LH}_{\text {EAKES6Lo }}$ is described as follows:

$$
\Gamma_{\text {EAKES6Lo }} \rightarrow \theta_{\text {EAKES6Lo }}\left[6 \mathrm{LH}_{\mathrm{EAKES6Lo}}\right]_{X} \operatorname{Honest}(\hat{Y}) \wedge \hat{Y} \neq \hat{X} \supset \phi_{6 L H}
$$

The formula $\theta[P]_{X} \phi$ means that if the precondition $\theta$ is true, the postcondition $\phi$ is true after actions $P$ executed by $X$. The postcondition $\phi_{6 L H}$ means that if $\hat{Y}$ is assumed to be honest, $\hat{X}$ is guaranteed that $\hat{Y}$ participates in EAKES6lo and each sending and receiving action happens in order after the actions executed by 6LHEAKES6Lo.

$$
\begin{aligned}
\phi_{6 L H} & \equiv \exists Y .((\operatorname{Send}(X, m s g 1)<\operatorname{Receive}(Y, m s g 1)) \\
& \wedge(\operatorname{Receive}(Y, m s g 1)<\operatorname{Send}(Y, m s g 2)) \\
& \wedge(\operatorname{Send}(Y, m s g 2)<\operatorname{Receive}(X, m s g 2))),
\end{aligned}
$$

where $m s g 1$ and $m s g 2$ are $<\left(I D_{6 L H}, I D_{6 L R}, T_{6 L H},\left(N_{6 L H}\right) K_{6 L H \leftrightarrow S E R}\right) K p r i_{6 L H}>$ and $<M A C 2,\left(I D_{6 L H}, I P v 6_{6 L H}, K p u b_{6 L H}, T_{S E R}, T_{E X P}\right) K p r i_{6 L H}>$ respectively.

Proof. A proof of the security property is shown as follows:

(1) AN3 $\theta_{\text {EAKES6Lo }}\left[\text { new } N_{6 L H}\right]_{X} \operatorname{Fresh}\left(X, N_{6 L H}\right)$

(2) FS1

$\operatorname{Fresh}\left(X, N_{6 L H}\right)[\operatorname{send} \hat{X}, \hat{Y}, \operatorname{Sig} 6 L H]_{X} \operatorname{FirstSend}\left(X, N_{6 L H 1},(\hat{X}, \hat{Y}, \operatorname{Sig} 6 L H)\right)$

(3) (1), (2), SEQ, P1

$$
\theta_{\text {EAKES6Lo }}\left[6 L H_{E A K E S 6 L o}\right]_{X} \operatorname{FirstSend}\left(X, N_{6 L H 1},(\hat{X}, \hat{Y}, \operatorname{Sig} 6 L H)\right)
$$

(4) (3), FS2 $\theta_{\text {EAKES6Lo }}\left[6 L H_{\text {EAKES6Lo }}\right]_{X} \operatorname{Receive}(Y,(\hat{X}, \hat{Y}, \operatorname{Sig} 6 L H)) \wedge \hat{Y} \neq \hat{X}$

$$
\supset \operatorname{Send}(X,(\widehat{X}, \widehat{Y}, \operatorname{Sig} 6 L H))<\operatorname{Receive}(Y,(\widehat{X}, \widehat{Y}, \operatorname{Sig} 6 L H))
$$

(5) AA1, P1, SEQ

$$
\theta_{\text {EAKES6Lo }}\left[6 L H_{E A K E S 6 L o}\right]_{X} \operatorname{Receive}(X,(\hat{Y}, \hat{X}, \operatorname{SigSER}, M A C 2))
$$


$\supset \operatorname{Has}\left(X, \operatorname{HASH}_{H K}\left(\operatorname{SigSER}, N_{6 L H}\right)\right)$

(6) (5), HASH4 $\theta_{\text {EAKES } 6 L o}\left[6 L H_{\text {EAKES } 6 L o}\right]_{\mathrm{X}} \exists Y, t 1$. Send $(Y, t 1)$

$\wedge$ Contains $\left(t 1, H_{A S H_{H K}}\left(\operatorname{SigSER}, N_{6 L H}\right)\right)$

(7) HON $\left(\operatorname{Honest}(\hat{Y}) \wedge \operatorname{Send}(Y, t 1) \wedge \operatorname{Contains}\left(t 1, \operatorname{HASH}_{H K}\left(\operatorname{SigSER}, N_{6 L H}\right)\right)\right)$

$\supset\left(\operatorname{New}\left(Y, N_{6 L H}\right) \vee\left(\operatorname{Receive}(Y, t 2) \wedge \operatorname{Contains}\left(t 2, N_{6 L H}\right)\right.\right.$

$<\operatorname{Send}(Y,(\hat{Y}, \hat{X},(\operatorname{SigSER}, M A C 2)))))$

(8) (1), (7), SEQ, AN1 $\theta_{\text {EAKES6Lo }}\left[6 \mathrm{LH}_{\text {EAKES6Lo }}\right]_{X}$ Honest $(\hat{Y}) \wedge \hat{Y} \neq \hat{X}$

$\supset(\exists Y$. Receive $(Y,(\hat{X}, \hat{Y}, \operatorname{Sig} 6 L H))$

$<\operatorname{Send}(Y,(\hat{Y}, \hat{X},(\operatorname{SigSER}, M A C 2)))$

(9) (5), (6), (8), SEQ $\theta_{\text {EAKES6Lo }}\left[6 \mathrm{LH}_{\text {EAKES6Lo }}\right]_{X} \operatorname{Honest}(\hat{Y}) \wedge \hat{Y} \neq \hat{X}$

$\supset \exists Y, t 1$. Send $(Y, t 1) \wedge \operatorname{Contains}\left(t 1, H_{A S H_{H K}}\left(\operatorname{SigSER}, N_{6 L H}\right)\right)$

$<\operatorname{Receive}(X,(\widehat{Y}, \hat{X}, \operatorname{SigSER}, M A C 2))$

$\supset \operatorname{Send}(Y,(\hat{Y}, \hat{X},(\operatorname{SigSER}, M A C 2)))$

$<\operatorname{Receive}(X,(\hat{Y}, \hat{X},(\operatorname{SigSER}, M A C 2)))$

(10) (4), (8), (9)

$$
\theta_{\text {EAKES6Lo }}\left[6 \mathrm{LH}_{\text {EAKES6Lo }}\right]_{X} \text { Honest }(\hat{Y}) \wedge \hat{Y} \neq \hat{X} \supset \phi_{6 L H}
$$

By now, each sending and receiving event of the principal $\widehat{X}, \hat{Y}$ have been proved to happen in order. Here, only the authentication property for $\hat{X}$ is presented. The proof for $\hat{Y}, \hat{Z}$ and $\hat{S}$, which can be derived in the similar way, are omitted.

\subsubsection{Formal Verification}

After the derivation of the logic correctness of the proposed protocol, the protocol needs to be further verified by AVISPA. AVISPA is a formal verification tool which can automatically validate the network security protocols and applications. Modules and a High Level Protocol Specification Language (HLPSL) are provided for specifying protocols and their security properties [69]. The HLPSL specification is automatically translated into a lower level language called Intermediate Format (IF) by AVISPA, which is used as the input of various back-ends. Two back-ends, constraint logic-based attack searcher (CL-Atse) [70] and on-the-fly model checker (OFMC) [71], 
are employed in the security analysis of EAKES6Lo due to the support of algebraic computations such as exclusive-or (XOR) and exponentiation. CL-Atse runs a protocol in all possible ways over bounded sessions, translating traces into constraints on the current intruder and environment state. Constraints are simplified by using rewriting and redundancy elimination techniques to model all the reachable states of the participants and to decide whether some security properties holds in all reachable states. For OFMC, an infinite tree is built based on the designed protocol, where the root is the initial system state and children represent the ways that a state can evolve. OFMC uses symbolic techniques to search for an attack by exploring tree in a demand driven way.

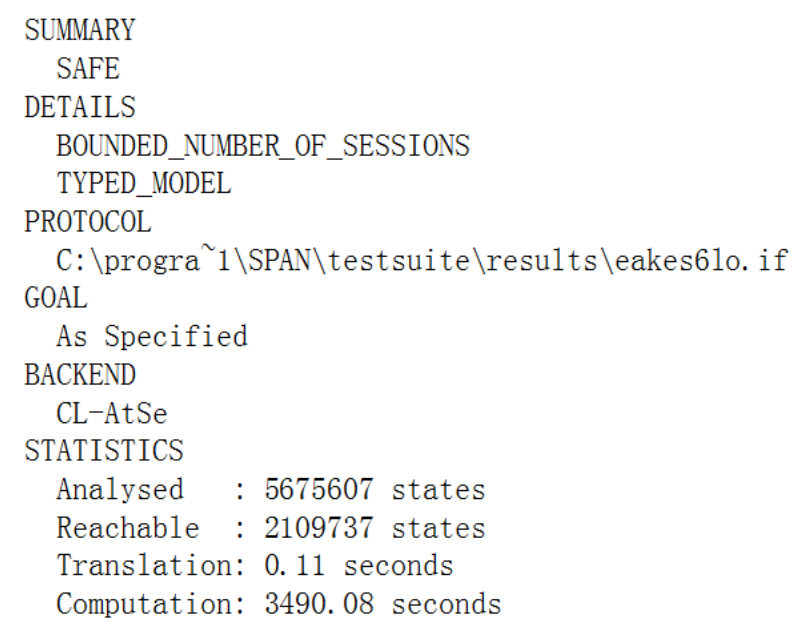

a) Output Results of EAKES6Lo using CL-Atse

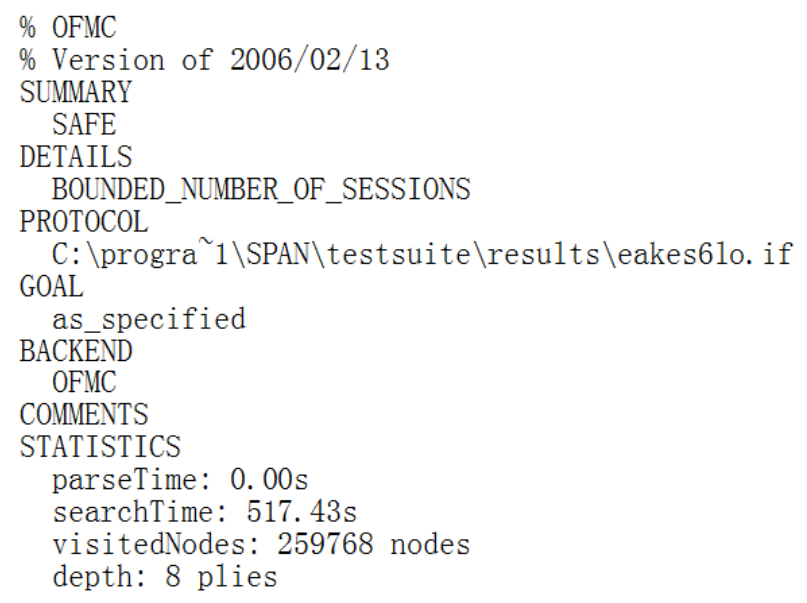

b) Output Results of EAKES6Lo using OFMC

Figure 3.5 Output Results of EAKES6Lo using AVISPA 
The proposed protocol is written in HLPSL and is analyzed by the two above mentioned back-ends. It is assumed that the intruder of the proposed protocol initially has the identities and public keys of all the participants in the proposed protocol, its own private key, symmetric keys shared with other entities, and other public functions. The security goals are defined in AVISPA that the confidential information are kept secret among the corresponding roles and the roles can be mutually authenticated. The output results of EAKES6Lo using CL-Atse and OFMC, as shown in Figure 3.5, demonstrate that the protocol is safe and proved to achieve the mutual authentication and goals as specified. In CL-AtSe, 5675607 states are analyzed and 2109737 states are reachable. It takes $0.11 \mathrm{~s}$ for protocol translation and $3490.08 \mathrm{~s}$ for algebraic computation. In OFMC, the protocol is analyzed with limited depth (the depth for the search is 8 ). The total number of nodes searched is 259768 , which takes $517.43 \mathrm{~s}$.

\subsubsection{Ability Against Malicious Attacks}

1) Replay Attacks.

A replay attack is an action to maliciously resend the obtained valid transmitted messages to a receiver by the attacker. By the proposed scheme, the replay attacks can be prevented by utilizing the timestamp mechanism. Suppose that an attacker has got one of the transmitted messages and sends it repeatedly. The receiver will compare the timestamp contained in the message with the receiving time. If the time difference is greater than the defined time threshold, the message will be considered as invalid and will be ignored. Even if the attacker can alter or delete the time stamp in the message, the attack cannot succeed since the message is transmitted with a signature or a MAC ${ }^{2}$ value.

2) Man-in-the-middle (MITM) Attacks.

An MITM attack is an attack that an attacker is involved in the communication between the two parties while they still believe that they are communicating directly with each other. Once the attack is successful, the attacker can eavesdrop, alter or delete the transmitted messages. Since the $\mathrm{MAC}^{2}$ and signature have been utilized for the protection in EAKES6Lo, the MITM attacks can be prevented as well. For example, 
although the attacker can obtain $I D_{6 L H}$ from the handover ticket, the signature value cannot be successfully changed because the attacker does not have the corresponding private key.

3) Impersonation Attacks.

By an impersonation attack, an adversary can disguise as one of the legitimate parties in the system. Since all the parties are assumed to have registered with the server before the deployment, if an adversary impersonates a 6LR in the network, the server will check its identity and its corresponding address to determine whether it is legitimate. When a $6 \mathrm{LH}$ moves from the area of 6LR1 to the area of 6LR2, the 6LH will initialize a handover request. After checking the validity of the handover ticket, the 6LR2 will tell 6LR1 that this 6LH has already left this area. The 6LR1 will delete $I D_{6 L H}$ from its member identity list. In this case, the attacker cannot disguise as a valid $6 \mathrm{LH}$ which still in the area of the previous router.

4) Sybil Attacks.

By a Sybil attack, an attacker can forge multiple identities. Like the impersonation attacks, the Sybil attacks can be prevented by the proposed scheme because when a $6 \mathrm{LH}$ is authenticated with the server, the server will check the registered ID and the corresponding address stored in the database. If the $6 \mathrm{LH}$ has multiple IDs using the same address, the server will consider it as a compromised device and send a warning message to the 6LER. The 6LER will then add the suspected 6LH to the black list and broadcast the list in the system.

5) Compromised Attacks.

By the proposed scheme, if a 6LR is compromised after the authentication, the attacker cannot threat the system because the secret nonce sent by a 6LH will be encrypted by the session key $K_{6 L H \leftrightarrow S E R}$. If a $6 \mathrm{LH}$ is compromised, the attacker can only obtain the messages sent to this $6 \mathrm{LH}$. Since the messages transmitted through the 6LER are either encrypted by the symmetric key or sent with a signature, the impacts of compromising the 6LER will be limited. The trusted server, which has enough storage and computing capacities, can execute more complicated security mechanisms to 
ensure its safety and it is assumed to be secure in EAKES6Lo. Table 3.3 lists the comparison of security properties between our solution and the SAKES scheme.

Table 3.3 Comparisons of Security Properties between EAKES6Lo and SAKES

\begin{tabular}{ccc}
\hline \hline & EAKES6Lo & SAKES \\
\hline Type of cryptosystem & Hybrid & Hybrid \\
Support for mobility & Yes & No \\
Against replay attack & Yes & Yes \\
Against MITM attack & Yes & Yes \\
Against impersonation attack & Yes & Yes \\
Against Sybil attack & Yes & Yes \\
Against compromised attack & Yes & No \\
Output result of AVISPA & Safe & Unsafe \\
\hline \hline
\end{tabular}

\subsection{Performance Evaluation}

In this section, our EAKES6Lo protocol is compared with the existing relevant protocols in terms of computation overhead and transmission overhead. The performance evaluation is conducted by MATLAB R2014b. The applied cryptographic algorithms employed in the simulation are Secure Hash Algorithm (SHA)- 256, Advanced Encryption Standard (AES)-128 and the Elliptic Curve Digital Signature Algorithm (ECDSA)-160. The setting of parameters is listed in Table 3.4.

\subsubsection{Computational Overhead}

In this section, the computation overhead of the proposed scheme is analyzed by MATLAB. The time of cryptographic operations used in the simulation refer to the 
Table 3.4 Setting of Parameters of EAKES6Lo

\begin{tabular}{cc}
\hline \hline Parameters & Value (bits) \\
IPv6 address & 128 \\
ID (MAC ${ }^{1}$ address) & 48 \\
Nonce & 32 \\
Timestamp & 32 \\
Hash value/MAC & 256 \\
\hline \hline
\end{tabular}

simulation results in $[72,73]$. The average time for hashing message using SHA-256 is $T_{H}=0.286 \mathrm{~ms} /$ byte. The average time for encrypting/decrypting message using AES128 Counter (CTR) mode is $T_{A E S}=0.177 \mathrm{~ms} /$ byte. The time needed for generation and verification of ECDSA are $T_{S}=520 \mathrm{~ms}$ and $T_{V}=1020 \mathrm{~ms}$ respectively. It is assumed that the time for generation of ECC private/public key pair is $T_{G}=550 \mathrm{~ms}$ and the time for calculating an ECDH key is $T_{E C D H}=500 \mathrm{~ms}$.

Thus, the total computational overhead of the EAKES6Lo and the SAKES are $6.24 \mathrm{~s}$ and $7.407 \mathrm{~s}$, respectively. Though EAKES6Lo is proved to be safe against the attacks as claimed, other types of malicious attacks as well as unknown attacks, which cannot be predicted, may cause the interruption of the execution of the protocol during the AKE phase. So, it is assumed that any type of attack may randomly occur at any step of the protocol execution during the AKE phase. The protocol cannot proceed if an attack successfully breaks it in the execution. With the increasing number of successful attacks, the average total time delay for a complete execution of the protocol will take longer. The comparison of the total time delay of the full protocol between EAKES6Lo and SAKES is shown in Figure 3.6 (c). The number of attacks used in the simulation is set to 10000 . It is shown that the EAKES6Lo scheme has lower computational overhead than that of the SAKES scheme. 
At each of the 6LHs, though the time cost of the EAKES6Lo is higher than that of the SAKES, the SAKES scheme is vulnerable to the compromised attacks because the 6LR can decrypt messages sent from the 6LH or the 6LER to compute the session key for the $6 \mathrm{LH}$ during the key establishment phase. To reduce the risks incurred by the compromised attacks, by the EAKES6Lo scheme, the 6LH will generate the session key itself. As a result, the computation cost of the $6 \mathrm{LH}$ is increased, but the ability to withstand the compromised attacks will be enhanced. The comparison of the total time delay of the 6LR between EAKES6Lo and SAKES is shown in Figure 3.6 (a). As for the server, the performance evaluation has been conducted on Intel(R) Core(TM) i53317U CPU@1.70 GHz with8 GB RAM and Windows 7 system. Since it has much more power than the wireless devices with limited resources, the total time delay of the EAKES6Lo scheme and the SAKES scheme are $26.26 \mathrm{~ms}$ and $39.46 \mathrm{~ms}$, respectively. The performance comparison between them is shown in Figure 3.6 (b).

\subsubsection{Transmission Overhead}

6LoWPAN devices are resource constrained devices regarding computation power, storage, and power supplier. Since the energy cost of transmission is much higher than the computation, the packet length should be as short as possible to extend the lifetime of the entire 6LoWPAN network. The energy consumption of the transmission also refers to the simulation results in [73]. The transmission overhead for receiving (RX) is $1.808 \mu \mathrm{J} /$ byte and transmitting with $0 \mathrm{dBm}$ power level (TX $0 \mathrm{dBm}$ ) is $1.672 \mu \mathrm{J} /$ byte. The energy cost of transmission of data within a LoWPAN using wireless communication is evaluated while the transmission using wired connection outside a LoWPAN is not considered. Thus, the total transmission overhead of the EAKES6Lo scheme is $1.405 \mathrm{~mJ}$, which is lower than that of $2.163 \mathrm{~mJ}$ by the SAKES scheme. The comparisons of the energy cost between EAKES6Lo and SAKES are shown in Figure $3.6(d)(e)$. 


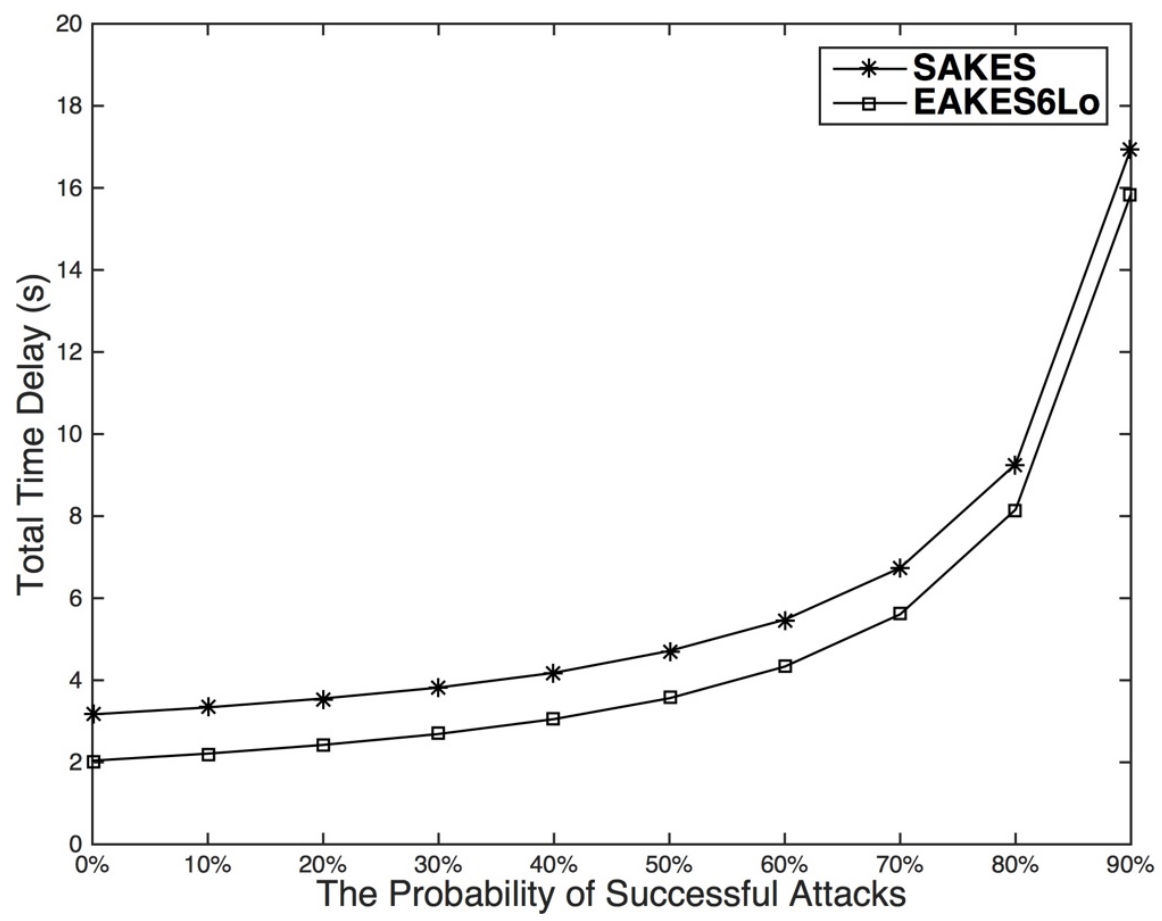

a) Total Time Delay of the 6LR

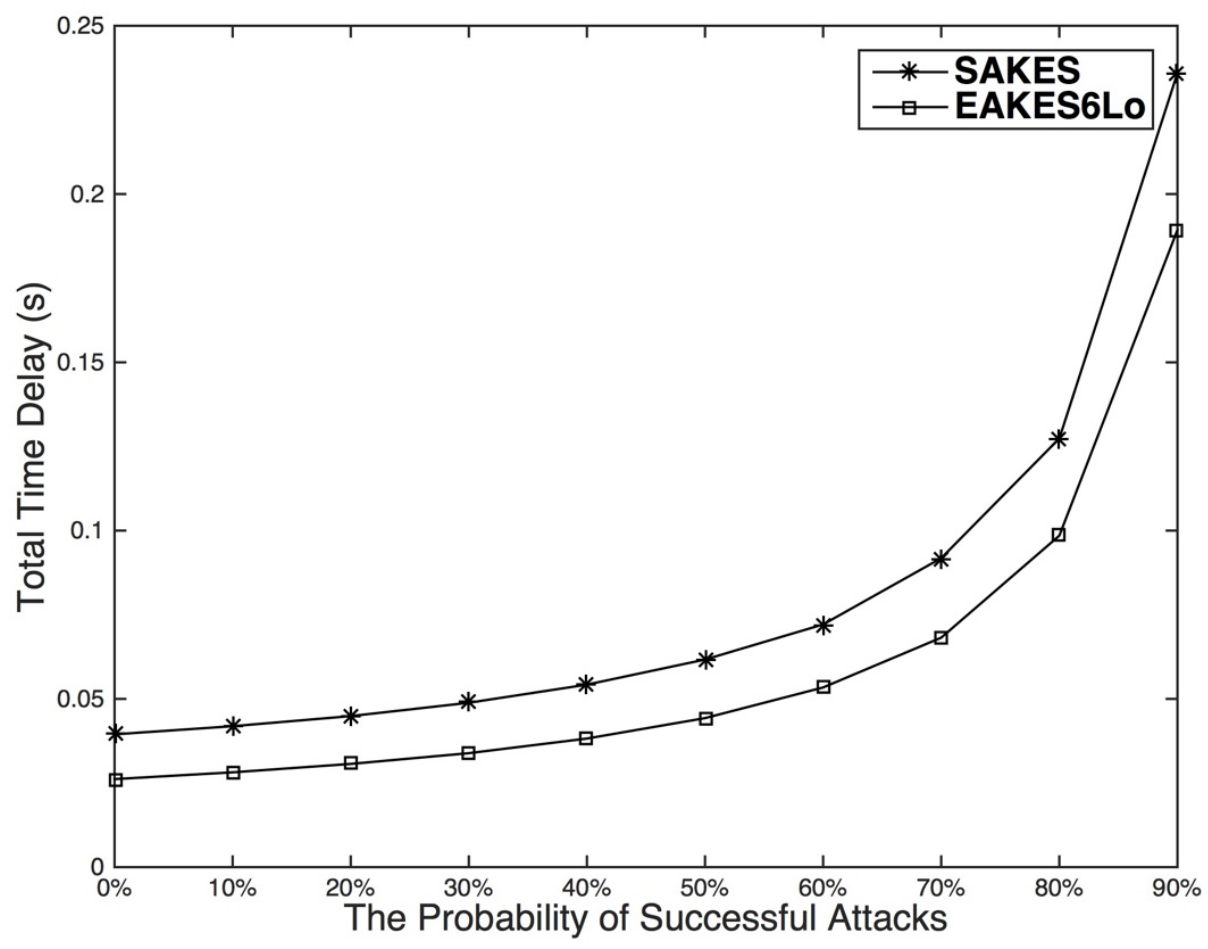

b) Total Time Delay of the Server 


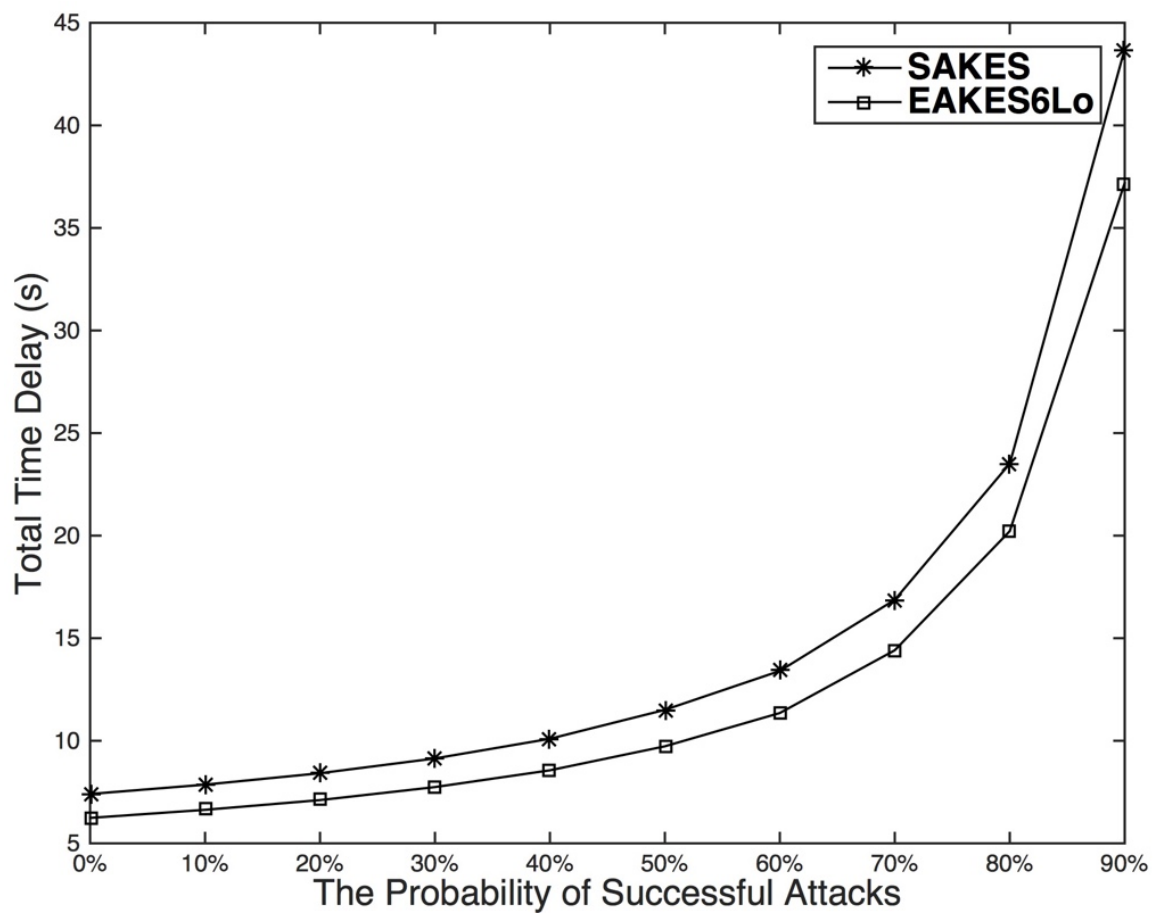

c) Total Time Delay of the Protocol

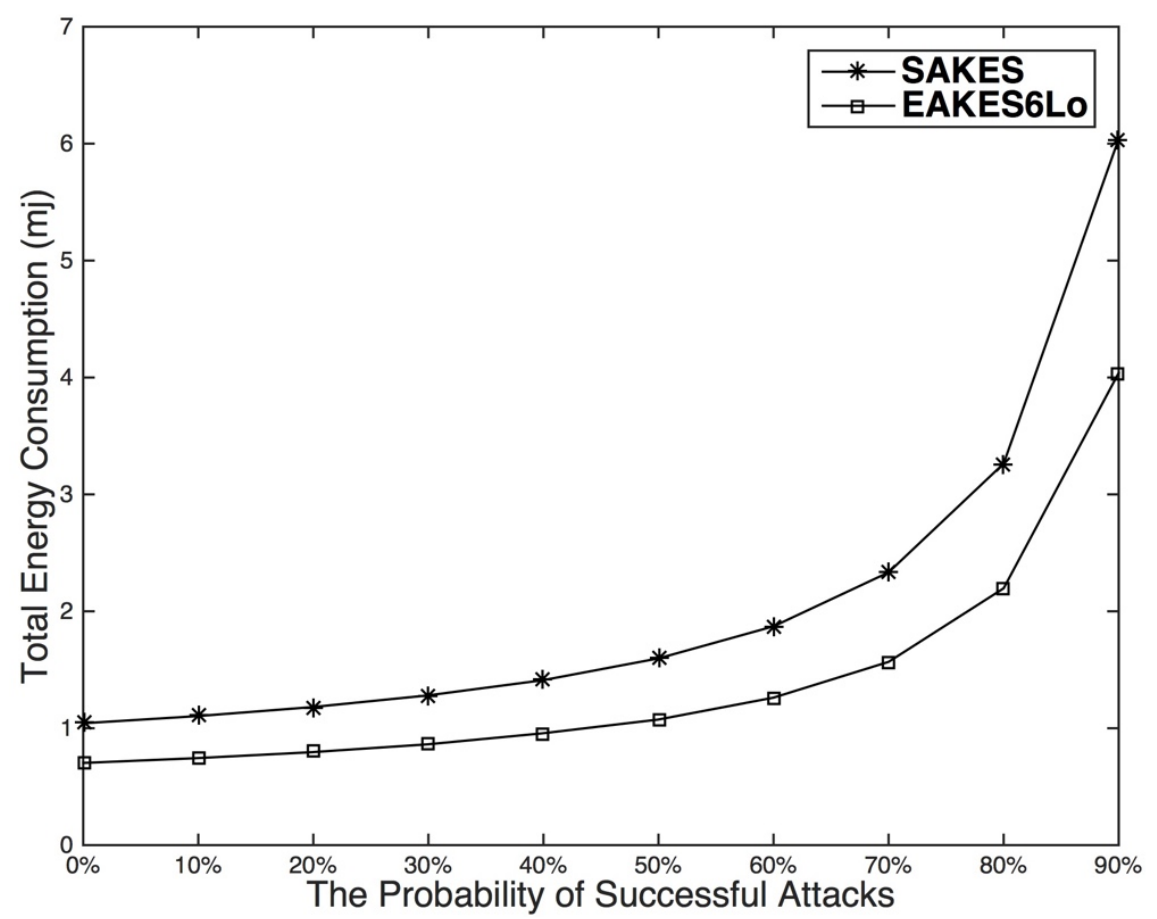

d) The Energy Consumption of the 6LR 


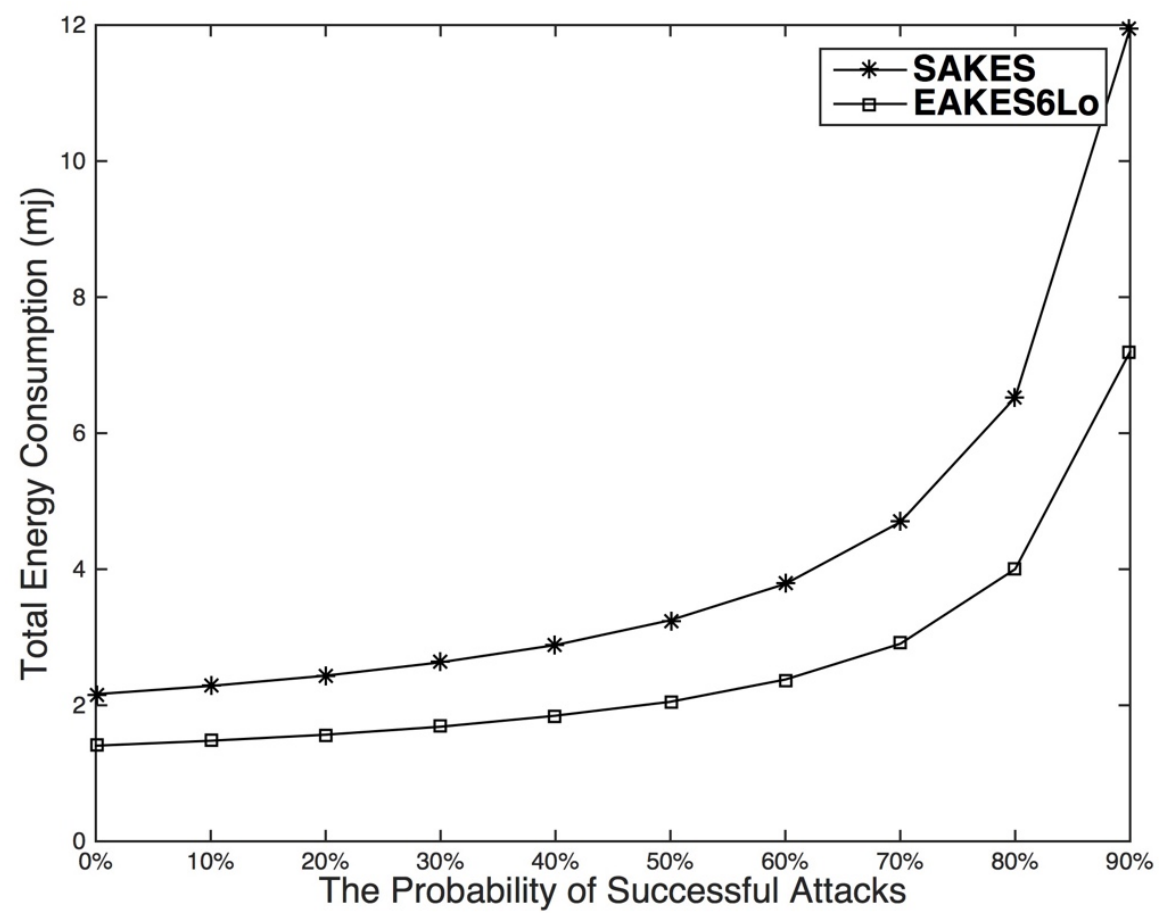

e) The Energy Consumption of the Protocol

Figure 3.6 Comparison of Performance Evaluation between EAKES6Lo and SAKES

\subsubsection{Comparison of Handover Processes}

A comparison of our proposed scheme EAKES6Lo with Fu et al. [63] and Zhang et al. [64] is given in Table 3.5. Though the handover authentication scheme [63] is proposed to achieve fast handover authentication using the handover ticket, the pairwise master key is shared between the MS and all BSs which is not secure nor scalable. If one of the BS is compromised by an adversary, the pairwise master key for devices that have stayed in this domain will all be exposed. The ticket-based authentication protocol [64] can also achieve fast handover authentication. However, the ticket can only be used when the mobile device roams in the service area of one of the neighbor APs. For example, if there are $m$ potential target APs for the mesh client, the AP has to generate $m$ tickets which is energy consuming for the resource constrained devices. 
Table 3.5 Comparisons of Ticket-based Schemes

\begin{tabular}{cccc}
\hline \hline & Fu et al. [63] & Zhang et al. [64] & EAKES6Lo \\
\hline Type of cryptosystem & Symmetric & Symmetric & Asymmetric \\
Requirement of neighbor graph & No & Yes & No \\
$\begin{array}{c}\text { Number of messages exchanged } \\
\text { during handover }\end{array}$ & 3 & 2 & 6 \\
$\begin{array}{c}\text { Number of tickets needed for } \\
\text { handover }\end{array}$ & 1 & $m^{*}$ & 1 \\
Same Pairwise Session keys & Yes & No & No \\
\hline \hline
\end{tabular}

* $m$ denotes the number of neighbor APs

\subsection{Summary}

To enhance the security functionality for the M2M communications in the 6LoWPAN networks, the EAKES6Lo scheme has been proposed in this chapter. A hybrid cryptography has been used to provide secure information exchanges among the $6 \mathrm{LHs}$, the 6LRs, the 6LER and the remote server. The security proof by the PCL can prove the logic correctness of the proposed scheme. The security analysis by the AVISPA has shown that the EAKES6Lo scheme is safe and can successfully prevent various malicious attacks including replay attacks, MITM attacks, impersonation attacks, and Sybil attacks, etc. The evaluation of the performance of the EAKES6Lo scheme demonstrates that EAKES6Lo has less computational overhead and transmission overhead and can support more efficient handovers. 


\section{Chapter 4 An Efficient and Secure PMIPv6-based Handover Scheme for 6LoWPAN Networks}

\subsection{Introduction}

In the previous chapter, we have introduced the EAKES6Lo, an authentication and key establishment scheme designed for the 6LoWPAN networks. Although the EAKES6Lo can provide security protections for MNs, the packet loss and the handover delay issues are inadequately considered. Besides, asymmetric encryption method produces much heavy computation overhead for the 6LoWPAN devices. To reduce the energy cost of the MNs and improve the handover efficiency, PMIPv6, a networkbased mobility management protocol as introduced in section 1.1.3, has been proposed by the IETF for resource constrained MNs to continuously access the network services while moving. The MAG, which the $\mathrm{MN}$ is attached to, is responsible for sending/receiving mobility-related control messages with the LMA instead of the MN so that PMIPv6 significantly reduces the signaling overhead of the MN and lower the handoff latency.

To enhance the performance of handover phase in terms of latency and packets loss, the fast handovers for PMIPv6 (FPMIPv6) protocol is proposed in [74]. To reduce packets loss, a bi-directional tunnel between the PMAG and the new MAG (NMAG) is established for packets buffering for the MN. FPMIPv6 performs better in term of packet loss compared to PMIPv6. However, more resources and larger buffer size of MAGs are required. To reduce the handover latency, a fast and localized PMIPv6 (FLPMIPv6) [75] is proposed. Although FLPMIPv6 ensures that the packet loss can be minimized, an MN with Media Independent Handover Function (MIHF) is required. Moreover, the signaling overhead between the MN and the MAG is higher than that of the PMIPv6 and FPMIPv6. A last packet marker based fast handover scheme [76] is

proposed to deal with the out of order packets problem. Due to frequent movement of the MNs, a large number of signals between the MAGs and the LMA are required to 
update the binding entry. To overcome this, a chaining based PMIPv6 (CB-PMIPv6) scheme [77] is proposed to reduce the signaling overhead during the handover by chaining the MAGs which the MN has attached to. However, it requires powerful MAGs to store the chained information of each MN when a significant number of MNs attach to the network. Although the schemes mentioned above have improved efficiency and significantly reduce the packets loss during the handover, the security issues have not been addressed.

To address the security with the mobility management, several PMIPv6-based authentication schemes [78-80] have been proposed for vehicular networks. However, very few of the proposals $[46,48]$ target at the security functions of the resource constrained IP-based devices. Although extensible authentication protocol (EAP) based authentication scheme can be used in PMIPv6, the computational overhead is much higher than what the 6LoWPAN resource constrained devices can afford. There are too many authentication messages exchanged between the $\mathrm{MN}$ and the authentication server in EAP-based authentication protocols, which result in long authentication latency. In this chapter, an efficient and secure PMIPv6-based handover (ESPH) scheme is designed for 6LoWPAN networks to provide more efficient and reliable mobility. With the consideration of the resource constrained 6LoWPAN devices, a hybrid cryptography method is employed to ensure the security supports for the MNs when roaming in the 6LoWPAN networks. To improve the security and the efficiency of the handover process, a key chain is generated for secure communication between the MNs and the MAGs to avoid the authentication on MNs each time when they are roaming among MAGs while still keeping security. The security proof, formal verification and the simulation results prove that the ESPH scheme could not only prevent many malicious attacks but also incur less computational overhead and handover latency. 


\subsection{System Background}

\subsubsection{System Architecture}

The 6LoWPAN system, as depicted in Figure 4.1, consists of a number of 6LoWPAN MNs (6MNs), some 6LoWPAN MAGs (6MAGs), a LMA and a authentication, authorization and accounting (AAA) server. The $6 \mathrm{MNs}$, also can be treated as the hosts, are mobile devices used for gathering sensory information. The 6MNs can move across different domains within the 6LoWPAN networks. The 6MAGs, which work as proxies, are responsible for detecting the movements of the $6 \mathrm{MNs}$ attached to it and sending binding updates to the LMA on behalf of the 6MNs. The LMA, which acts as a HA in PMIPv6, maintains the binding cache entries for currently registered $6 \mathrm{MNs}$ and forwards the data packets for the $6 \mathrm{MN}$ and the correspondent node. The LMA assigns the $6 \mathrm{MN}$ 's home network prefix to the link between the $6 \mathrm{MN}$ and the $6 \mathrm{MAG}$, from which the $6 \mathrm{MN}$ can configure its IP addresses. The AAA server is responsible for authenticating the $6 \mathrm{MNs}$ and storing the policy files which are sets of configuration information of the 6MNs.

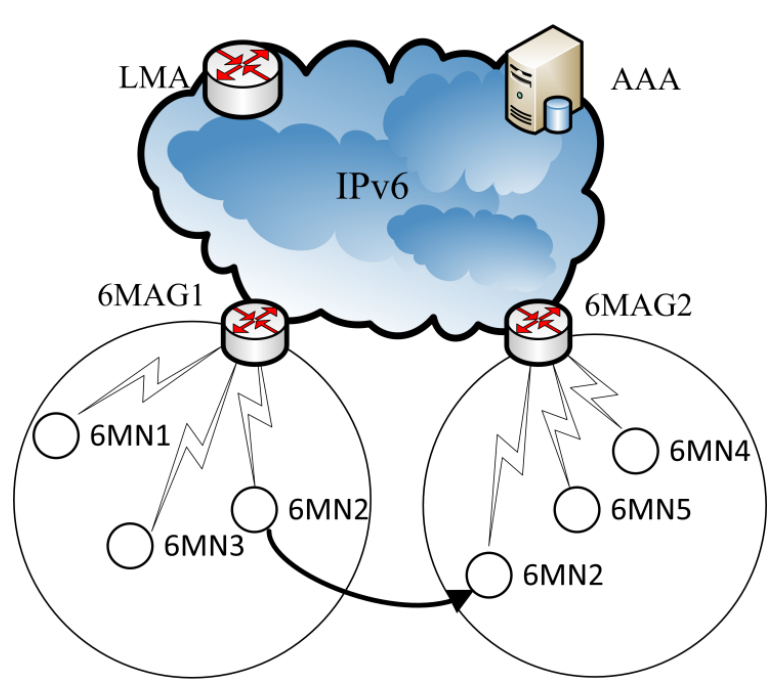

Figure 4.1 6LoWPAN System Architecture of ESPH 
The LMA and the AAA server are assumed to be trusted backbone entities. The 6MAGs are connected with the LMA through wired links with high bandwidth and low bit error rates. Thus, the 6MAGs and the LMA are mandatory to implement IPsec for protecting the PMIPv6 signaling messages. The IPsec is a security protocol to provide secure E2E communications at the IP layer. By the IPsec, the LMA can effectively authenticate the 6MAGs within its scope. Besides, the Internet Key Exchange Protocol version 2 (IKEv2) is implemented to help the 6MAGs, the LMA and the AAA server to establish security associations which are used for pre-sharing the symmetric keys among them. Although the IPsec and the IKEv2 are mandatory with IPv6, they may not be suitable for the resource constrained $6 \mathrm{MNs}$ because these devices may not have enough capability to perform all of the functions.

\subsubsection{Attack Model}

The 6LoWPAN devices with limited resources are usually placed in unattended and accessible places without better supervision and more vulnerable to various attacks compare to traditional equipment which has enough resources. The threat model to ESPH is based on the Dolev-Yao intruder model [65] which is previously described in section 3.2.3.

\subsection{Review of the SPAM Scheme}

\subsubsection{Details of the SPAM Scheme}

The SPAM scheme is a state-of-the-art solid solution to provide PMIPv6-based authentication and key establishment for resource constrained devices. Therefore, the details of the operations of the SPAM will be reviewed in this section. In the SPAM's architecture, since the MAGs, the LMA and the AAA server are connected through wired network, the IPsec and the IKEv2 can be implemented to negotiate the security associations with each other and protect their communications. Thus, some symmetric keys can be pre-shared to assist the authentication procedure. Besides, each device stores the security parameters in its smart card which is tamper-resistant. That is, each 
user needs to input the identifier and password which will be verified by the smart card for accessing the mobile Internet. The notations used are listed in Table 4.1.

Table 4.1 Notations Used in Chapter 4

\begin{tabular}{|c|c|}
\hline Notation & Description \\
\hline$s v$ & Secret key generated by the AAA server \\
\hline$I D_{x}$ & Identity of $x$ \\
\hline$P W_{x}$ & Password of $x$ \\
\hline$P S K$ & Pre-shared symmetric group key \\
\hline$N, r$ & Nonce \\
\hline$r t$ & Root value \\
\hline$T_{i}$ & Timestamp \\
\hline$S K_{x \leftrightarrow y}$ & Session key established between $x$ and $y$ \\
\hline$s_{x}$ & Private key of $x$ \\
\hline$P u b_{x}$ & Public key of $x$ \\
\hline$k_{r}$ & Registration key \\
\hline$H_{0}, H_{1}$ & One-way hash function. \\
\hline$H M A C$ & Hash-based MAC ${ }^{2}$ \\
\hline$E_{K}(M)$ & Message $M$ is encrypted with the key $K$ \\
\hline$D_{K}(C)$ & Ciphertext $C$ is decrypted with the key $K$ \\
\hline
\end{tabular}

Before an MN is deployed into a PMIPv6 domain, a smart card is inserted into the $\mathrm{MN}$ by the user. After that, this MN should register with the AAA server through a secure channel. The registration phase has three steps which are described as follows. A password $P W_{M N}$ is chosen by the user and will be sent with its identification to the 
server. Upon receiving the registration information from the MN, the AAA server will compute the security parameters by $c 1=H_{0}\left(I D_{M N}|| s v\right), c 2=H_{0}\left(P W_{M N}\right) \oplus c 1, c 3=$ $E_{P S K}\left(I D_{A A A} \| s v\right), c 4=H_{0}\left(I D_{A A A} \| s v\right)$, and $c 5=H_{0}(s v)$. These parameters will be stored in the MN's smart card for further usage.

If an $\mathrm{MN}$ is authenticated, it can access the PMIPv6 network. When it roams from the domain of the PMAG to a domain of an NMAG, the handover procedure will be performed. The steps of the proposed handover procedure are described as follows.

- When detecting a handover movement of an MN, the PMAG sends a proxy handover initiate (HI) message $<E_{P S K}\left(I D_{M N}\right)>$ to the NMAG.

- If the proxy HI message can be correctly decrypted by the NMAG, the data packets will be buffered by the NMAG. An encrypted message < $I D_{M A G}, E_{P S K}\left(B P B U\left\|N_{1}\right\| H_{0}\left(N_{1} \| I D_{M A G}\right)>\right.$ will be sent to the LMA to inform that the $\mathrm{MN}$ is going to perform the handover, where BPBU is a bicasting proxy binding update (PBU) message.

- The NMAG will be verified when the LMA receives the BPBU message. If the NMAG is legal, a session key $S K_{L M A \leftrightarrow N M A G}$ will be generated as $H_{0}\left(N_{1} \| N_{2}\right)$, the binding state of the corresponding MN will be updated and a bicasting tunnel is established between the LMA and the NMAG. After that, the downstream data packets will be transmitted to both the PMAG and the NMAG. Then, a bicasting proxy binding acknowledgement (PBA) (BPBA) message $<I D_{L M A}, E_{P S K}\left(B P B A\left\|N_{1} \oplus 1\right\| N_{2} \| H_{0}\left(N_{2} \| I D_{L M A}\right)\right)>$ is sent back to the NMAG to acknowledge the new binding.

- After successfully decrypting the ciphertext sent from the LMA, the NMAG will first check the validity of the information. If it is valid, the session key $S K_{L M A \leftrightarrow N M A G}$ is calculated as $H_{0}\left(N_{1} \| N_{2}\right)$. Otherwise, the message will be ignored. The bi-directional tunnel is established between the PMAG and the NMAG for transferring the data packets belonging to the approaching MN. Afterwards, a proxy handover acknowledgement (HACK) message is sent to the PMAG in response to the proxy HI message protected by the pre-shared symmetric key. 
- When the MN roams to the domain of the NMAG, it sends a router solicitation (RS) message to the NMAG including $<A I D_{M N}, c 3, E_{c 4}\left(R S\left\|A U T H_{M N}\right\|\right.$ $\left.N_{3}\right)>$ where the alias ID $A I D_{M N}=I D_{M N} \oplus H_{0}\left(c 5|| N_{3}\right)$ and the authentication vector $A U T H_{M N}=H_{0}\left(c 1|| N_{3}\right)$.

- Upon receiving the RS message from the newly attached MN, the NMAG decrypts $c 3$ using its pre-shared key PSK and obtains $I D_{A A A}$ and $s v$. The authentication vector will be correctly obtained from the ciphertext if $c 4$ is equal to the hash value of $I D_{A A A}$ and $s v$. The original identification of $\mathrm{MN}$ is calculated by $A I D_{M N} \oplus H_{0}\left(c 5 \| N_{3}\right)$. Once the $\mathrm{MN}$ is authenticated, a PBU message $<E_{S K_{L M A \leftrightarrow N M A G}}\left(N_{2} \oplus 1\right)>$ is going to be sent to the LMA.

- If the PBU message is successfully retrieved, the LMA will then stop the bicasting and update the binding state of the $\mathrm{MN}$. In response to the PBU message, a PBA message is replied by the LMA and sent to the NMAG.

- If the validity of the information is verified, a new nonce $N_{4}$ is generated to calculate the session key $S K_{M N \leftrightarrow N M A G}$ as $H_{0}\left(N_{3} \| N_{4}\right)$ and the router advertisement (RA) message $<I D_{N M A G}, E_{C 4}\left(N_{3} \oplus 1|| N_{4}|| H_{0}\left(I D_{N M A G} \| N_{4}\right)\right)>$ will be sent as a response to the $\mathrm{MN}$. If the $\mathrm{MN}$ is still in the domain of the same LMA, it will obtain the original address. Otherwise, a new global IPv6 address is assigned with a new and unique network prefix. On receipt of the RA message, the $\mathrm{MN}$ verifies the $\mathrm{MAG}$ by checking if $N_{3} \oplus 1$ and $H_{0}\left(I D_{N M A G} \| N_{4}\right)$ are correct. After that, a session key is computed in the same way as the MAG. Thus, the session key $S K_{M N \leftrightarrow N M A G}$ is established between the MN and the NMAG.

- An encrypted message $<E_{S K_{M N} \leftrightarrow N M A G}\left(N_{4} \oplus 1\right)>$ is sent to the NMAG for the confirmation of the nonce $N_{4}$. The message can be accurately decrypted if the session key computed by the MN and the NMAG are the same. Then, the MN sends the last sequence number of the packet sent by the PMAG in order to continuously receive the data packets. The buffered data packets will be sent to this $\mathrm{MN}$ in sequence from the NMAG. 


\subsubsection{Vulnerabilities of the SPAM Scheme}

As pointed out in [47], though the SPAM scheme has some advantages to prevent the packet loss, it has several fatal drawbacks.

- If an adversary eavesdrops on the link and obtains the authentication request message $<A I D_{M N}, c 3, E_{c 4}\left(A U T H_{M N} \| N_{3}\right)>$ sent by the MN, the NMAG cannot distinguish whether the nonce $N_{3}$ is fresh or not when the adversary resends the same information in the RS message to the NMAG. Besides, if key $c 4$ is stolen, the adversary can impersonate a legal MN and establish the session key with MAGs which the MN is not attached to.

- Since the key PSK is pre-shared among all the MAGs and the LMA, the group key $P S K$ can be obtained by the adversary if one MAG is compromised. Thus, $I D_{A A A}$ and the secret key $s v$ can be recovered from $c 3$ by using $P S K$. This drawback is fatal to the whole system because the adversary can perform like a AAA server to generate fake $c 1, c 2, c 3, c 4$ and $c 5$ by inputting a random identification $I D^{\prime}$ and the password $P W^{\prime}$. Therefore, a compromised MAG can assign the security parameters to a large number of MNs which may do not exist. As a result, a lot of legal PBU messages are sent to the LMA which may lead to DoS attack.

In addition to the impact of the compromised MAGs, a registered but dishonest $\mathrm{MN}$ (DMN) can also launch malicious attacks because the security parameters $c 3, c 4$ and $c 5$ assigned to each $\mathrm{MN}$ are the same. For example, if a DMN eavesdrops the messages transmitted between a legal MN and the MAG and captures the messages during the authentication phase, the real ID of a legal MN can be retrieved by the equation $I D_{M N}=A I D_{M N} \oplus H_{0}\left(c 5|| N_{3}\right)$. Moreover, the DMN is able to calculate the session key built between the legal $\mathrm{MN}$ and the MAG using $N_{3}$ and $N_{4}$ which are obtained from the messages encrypted with $c 4$. 


\subsection{The ESPH Scheme}

\subsubsection{Motivation}

To overcome the shortcomings of SPAM, an enhanced handover scheme is designed with novel ideas. To provide a more secure and efficient handover procedure for 6LoWPAN networks, the $6 \mathrm{MN}$ and the LMA generate a same key chain based on the 6MN's movement for secure communication. Thus, the AAA server only needs to authenticate the $6 \mathrm{MN}$ during a certain period when the $6 \mathrm{MN}$ roams in the network which significantly reduces the handover delay. Besides, considering the resource constraints on $6 \mathrm{MNs}$, only symmetric cryptography and simple hash function are used to protect the transmitted messages. As for the 6MAGs, the LMA and the AAA server, since they are linked through wired links and have enough resources and capabilities, more complicated encryption methods are employed for providing a more secure protection.

\subsubsection{Details of the ESPH Scheme}

To enhance the security functionality of the 6LoWPAN networks, the ESPH is designed for secure PMIPv6 in 6LoWPAN networks. It consists of three phases: 1) registration phase, 2) initial AKE phase and 3) handover phase. The notations used in this scheme are listed in Table 4.1. It is assumed that all the devices have a unique 64bit IID or 16-bit short address within a single LoWPAN and the links among the 6MAGs, the LMA and the AAA server are connected using wired cable which can be protected by the existing IPsec. Each legitimate device cannot leak the information to the third party. Besides, the backbone LMA and the authentication server are trustworthy.

1) Registration Phase

Before the deployment of a new 6LoWPAN device, a parameter list $\left\{p, a, b, G, n, h, H_{1}\right\}$ is published by the system through the following steps:

- $\quad$ Choose a $k$-bit prime number $p$ specifying the finite field $F_{p}$. 
- Choose two coefficients $a$ and $b$ specifying an elliptic curve $E / F_{p}$ which is defined by the equation $y^{2}=x^{3}+a x+b \bmod p$.

- Choose a base point $G=(x, y)$ on $E\left(F_{p}\right)$ that generates the subgroup whose prime order is $n$ and cofactor is $h$.

- Let hash function $H_{1}:\{0,1\}^{*} \rightarrow F_{p}^{*}$.

- After publishing the parameter list $\left\{p, a, b, G, n, h, H_{1}\right\}$, a public/private key pair can be generated based on these parameters. For example, a secret number $s_{i} \in[1, n-1]$ is selected to be the private key of a $6 \mathrm{MN} i$ and $P u b_{i}\left(=s_{i} G\right)$ is its public key.

By the ESPH, prior to accessing the network services, each 6MN needs to perform the registration procedure with the AAA server through a secure channel. A unique identification $I D$ and a proper password $P W$ are chosen to be inputted to the tamperresistant smart card of the $6 \mathrm{MN}$ for registration. The smart card calculates a pseudo ID by $P I D=H_{1}\left(I D \| r_{0}\right)$ where $r_{0}$ is a newly generated nonce. The $6 \mathrm{MN}$ first sends a registration request to the server with the real ID, $P W, P I D$ and the nonce $r_{0}$. After checking that this node has not been registered before, the server will send a signature $\sigma=E_{S_{A A A}}\left(H\left(P I D \| I D_{A A A}\right)\right)$ and a key $k_{r}$ for registration in response to the request and store $<I D, P I D, H_{1}\left(I D|| P W \| r_{0}\right), r_{0}, \sigma>$ in the database which will be further used in the following phases. When receiving the key $k_{r}$ and the signature, the $6 \mathrm{MN}$ stores $<$ $I D, P W, P I D, r_{0}, k_{r}, \sigma>$ in its smart card. The registration steps of ESPH are depicted in Figure 4.2.

6MN

AAA

Enters $I D$ and $P W$

$$
\begin{aligned}
& P I D=H\left(I D \| r_{0}\right) \quad<I D\|P W\| P I D \| r_{0}> \\
& P I D=H\left(I D \| r_{0}\right) \\
& \left.<\sigma, k_{r}\right\rangle \quad \sigma=E_{S_{A A A}}\left(H\left(P I D \| I D_{A A A}\right)\right) \\
& I D, P W, P I D, r_{0}, \sigma, k_{r} \quad I D, P I D, H_{1}\left(I D\|P W\| r_{0}\right), r_{0}, \sigma
\end{aligned}
$$

Figure 4.2 Registration Phase of ESPH 


\section{2) Initial AKE Phase}

In this phase, the $6 \mathrm{MN}$, the $6 \mathrm{MAG}$, the LMA and the AAA server will exchange eight messages to achieve a mutual authentication as shown in Figure 4.3. The details of the initial authentication process are described as follows.

$$
\begin{aligned}
& \text { 6MN 6MAG LMA } \quad \text { AAA } \\
& \mathrm{RS}:<P I D\|T 1\| M s g \| u 1=\sigma \oplus H M A C_{k_{r}}(P I D|| T 1 \| M s g)> \\
& <P I D\left\|I D_{6 M A G}\right\| T 1\|T 2\| M s g \| u 2> \\
& u 2=\sigma \oplus H M A C_{S K_{6 M A G \leftrightarrow L M A}}\left(P I D\left\|I D_{6 M A G}\right\| T 1\|T 2\| M s g\right) \\
& <P I D\left\|I D_{6 M A G}\right\| I D_{L M A}\|T 1\| T 3\|M s g\| u 3> \\
& u 3=\sigma \oplus H M A C_{S K_{L M A \leftrightarrow A A A}}\left(P I D\left\|I D_{6 M A G}\right\| I D_{L M A}\|T 1\| T 3 \| M s g\right) \\
& <P I D\|T 4\| u 4=\left(r t 1\|r t 2\| r t 1^{I D}\left\|r t 2^{I D}\right\| H\left(r t 1\|r t 2\| r t 1^{I D} \| r t 2^{I D}\right)\right. \\
& \bigoplus H M A C_{S K_{L M A \leftrightarrow L M A}}(P I D \| T 4)> \\
& <P I D\|T 5\| u 5=\left(\text { key }_{0} \| H\left(\text { key }_{0}\right) \oplus H M A C_{S K_{6 M A G \leftrightarrow L M A}}(P I D \| T 5)\right)> \\
& \text { PBU } \\
& \text { PBA } \\
& \mathrm{RA}:<E_{\text {key }}\left(P I D\left\|I D_{6 M A G}\right\| T 6 \| \overleftarrow{\text { Prefix })>}\right.
\end{aligned}
$$

Figure 4.3 Initial Authentication Phase of ESPH

When a new 6MN first joins in a 6LoWPAN, it will send a RS message that requests a $6 \mathrm{MAG}$ to assign a network prefix for configuring the IPv6 address. The RS message includes the following information. Two numbers $r_{1}$ and $r_{2}$ are randomly chosen for the computation of the key chain. The key $S K_{6 M N \leftrightarrow A A A}$ is calculated using $S K_{6 M N \leftrightarrow A A A}=H_{1}\left(I D \oplus r_{0}|| P I D \| T 1\right)$. A $\mathrm{MAC}^{2}$ will be calculated by the equation $M A C 1=H M A C\left(k_{r}, P I D|| T 1|| M s g\right)$. The $6 \mathrm{MN}$ sends $<P I D\|T 1|| M s g\| u 1=$ $\sigma \bigoplus M A C 1>$ as message 1 to the nearest 6MAG where $M s g=E_{S K_{6 M N \leftrightarrow A A A}}\left(r_{1}\left\|r_{2}\right\|\right.$ $P W \| H_{1}\left(r_{1} \| r_{2}\right)$.

In order to reduce the time used in the AKE phase, the key chain that will be used in handover process, is calculated in advance. The root values $r t_{1}$ and $r t_{2}$ are computed by $r t_{1}=H_{1}\left(P I D \| r_{1}\right)$ and $r t_{2}=H_{1}\left(P I D \| r_{2}\right)$, respectively. Thus, the $6 \mathrm{MN}$ 
can produce the initial session key $k e y_{0}=H_{1}\left((r t 1 \| x)\|0\|\left(r t 2^{I D} \| y\right) \| 0\right)$ based on the coordinate of the $6 \mathrm{MAG}$.

The $6 \mathrm{MAG}$, which receives the RS message from the $6 \mathrm{MN}$, will check the validity of the timestamp by $|T 1-T|<\Delta T$. If it is valid, the hash value will be calculated using the received information by $\operatorname{HMAC}\left(k_{r}, P I D^{\prime} \| T 1^{\prime}|| M s g^{\prime}\right)$ and the signature can be obtained by $\sigma^{\prime}=\sigma \bigoplus M A C 1 \oplus M A C 1^{\prime}$ which can be further verified using the public key of the AAA server. If the equation is satisfied, the $I D_{6 M A G}$ and a new timestamp will be included in the message transmitted to the LMA. The message < $P I D, I D_{6 M A G}, T 1, T 2, M s g, u 2=\sigma \oplus M A C 2>$ will be sent to the LMA as message 2, where $M A C 2=\operatorname{HMAC}\left(S K_{6 M A G \leftrightarrow L M A}, P I D\left\|I D_{6 M A G}\right\| T 1\|T 2\| M s g\right)$.

After receiving the message 2, the LMA will verify it in the same way as the 6MAG. A new message $<P I D, I D_{6 M A G}, I D_{L M A}, T 1, T 3, M s g, u 3=\sigma \bigoplus M A C 3>$ will be sent to the AAA server as message 3 where $M A C 3=H M A C\left(S K_{L M A \leftrightarrow A A A}\right.$, PID $\left.\left\|I D_{6 M A G}\right\| I D_{L M A}\|T 1\| T 3 \| M s g\right)$.

Upon receiving message 3, the AAA server will first check the validity of the message and verify the signature. After that, if the $6 \mathrm{MN}$ has been registered with the server before, the symmetric key $S K_{6 M N \leftrightarrow A A A}$ is calculated by $S K_{6 M N \leftrightarrow A A A}=$ $H_{1}\left(I D \oplus r_{0}\|P I D\| T 1\right)$ according to the stored data corresponding to the pseudo ID. The encrypted information $<r_{1}\left\|r_{2}\right\| P W \| H_{1}\left(r_{1} \| r_{2}\right)>$ can be decrypted if the symmetric key is correct. The received password can be verified if the value of $H_{1}\left(I D^{\prime}\left\|P W^{\prime}\right\| r_{0}{ }^{\prime}\right)$ is equal to the one stored in the AAA server. Two root value $r t_{1}$ and $r t_{2}$ are calculated through the function $r t_{1}=H_{1}\left(P I D \| r_{1}\right)$ and $r t_{2}=H_{1}\left(P I D \| r_{2}\right)$ respectively and will be sent to the LMA for session key establishment. The AAA server sends out $<P I D$, $T 4, u 4=\left(r t_{1}\left\|r t_{2}\right\| r t_{1}^{I D}\left\|r t_{2}^{I D}\right\| H_{1}\left(r t_{1}\left\|r t_{2}\right\| r t_{1}^{I D} \| r t_{2}^{I D}\right)\right) \oplus M A C 4>$ as message 4 to inform the LMA that the $6 \mathrm{MN}$ is legitimate where $M A C 4=\operatorname{HMAC}\left(S K_{L M A \leftrightarrow A A A}\right.$, $P I D \| T 4)$. If the $6 \mathrm{MN}$ has not been registered, a warning message will be sent out that the $6 \mathrm{MN}$ is an illegitimate device.

The LMA, which receives the message 4 from the AAA server, will first check the validity of the timestamp (e.g. $|T 4-T|<\Delta T$ ). After the verification, the LMA will 
use the received $6 \mathrm{MN}$ 's identity, timestamp, the key shared between the LMA and the AAA server to compute the $\mathrm{MAC}^{2}$ value for extracting the root key. The two root keys $r t_{1}^{\prime}$ and $r t_{2}^{\prime}$ can be derived by $\left(r t_{1}^{\prime}\left\|r t_{2}{ }^{\prime}\right\| r t_{1}^{I D^{\prime}}\left\|r t_{2}^{I D \prime}\right\| H_{1}\left(r t_{1}\left\|r t_{2}\right\| r t_{1}^{I D} \| r t_{2}^{I D}\right)\right)$ $\bigoplus H M A C\left(S K_{L M A \leftrightarrow A A A}, P I D \| T 4\right) \oplus H M A C\left(S K_{L M A \leftrightarrow A A A}, P I D^{\prime} \| T 4^{\prime}\right)$. The four root keys are successfully obtained if $H_{1}\left(r t_{1}^{\prime}\left\|r t_{2}^{\prime}\right\| r t_{1}^{I D^{\prime}} \| r t_{2}^{I D \prime}\right)=H_{1}\left(r t_{1}\left\|r t_{2}\right\| r t_{1}^{I D} \|\right.$ $\left.r t_{2}^{I D}\right)$. Then, the key is calculated $k e y_{0}=H_{1}\left(\left(r t_{1} \| x\right)\|0\|\left(r t_{2}^{I D} \| y\right) \| 0\right)$. After knowing the corresponding $6 \mathrm{MN}$ is legal, a new message $<P I D\|T 5\| u 5=$ $\left(\right.$ key $\left._{0} \| H_{1}\left(k e y_{0}\right)\right) \oplus M A C 5>$, where $M A C 5=H M A C\left(S K_{6 M A G \leftrightarrow L M A}, P I D \| T 5\right)$, will be sent out as message 5 to the $6 \mathrm{MAG}$ to which the $6 \mathrm{MN}$ is attached.

When receiving the message 5 from the LMA, the 6MAG recovers the session key $k e y_{0}$ from the received the data. After verifying the validity of the session key, the 6MAG will send a PBU request as message 6 to the LMA for binding. A PBA will be sent back to this $6 \mathrm{MAG}$ as message 7 in which a unique network prefix is assigned to the $6 \mathrm{MN}$ by the LMA. The $6 \mathrm{MAG}$ will send a RA in response to the RS which the $6 \mathrm{MN}$ sends at first. The 6MN's pseudo ID, 6MAG's ID, network prefix and the timestamp are encrypted with the session key $k e y_{0}$. The message $<E_{k e y_{0}}(P I D \|$ $I D_{6 M A G}\|T 6\|$ Prefix) $>$ is included with the RA message sent to the $6 \mathrm{MN}$.

The $6 \mathrm{MN}$, which finally receives the RA message from the $6 \mathrm{MAG}$, will derive the network prefix from the encrypted information by using the calculated session key. If the corresponding key is valid, the message can be obtained correctly. Thereafter, the $6 \mathrm{MN}$ and the $6 \mathrm{MAG}$ can communicate using the key $k e y_{0}$ in a valid session.

3) Handover Phase

Most existing schemes require a full authentication procedure performed during each handover procedure resulting in a longer handover latency and a heavier workload. To reduce the handover latency, the authentication process during the handover phase, which combines the hash chain with the direction of the movement of the $6 \mathrm{MN}$, is much simplified compared to the existing schemes while still retaining security. The handover phase is depicted in Figure 4.4. 


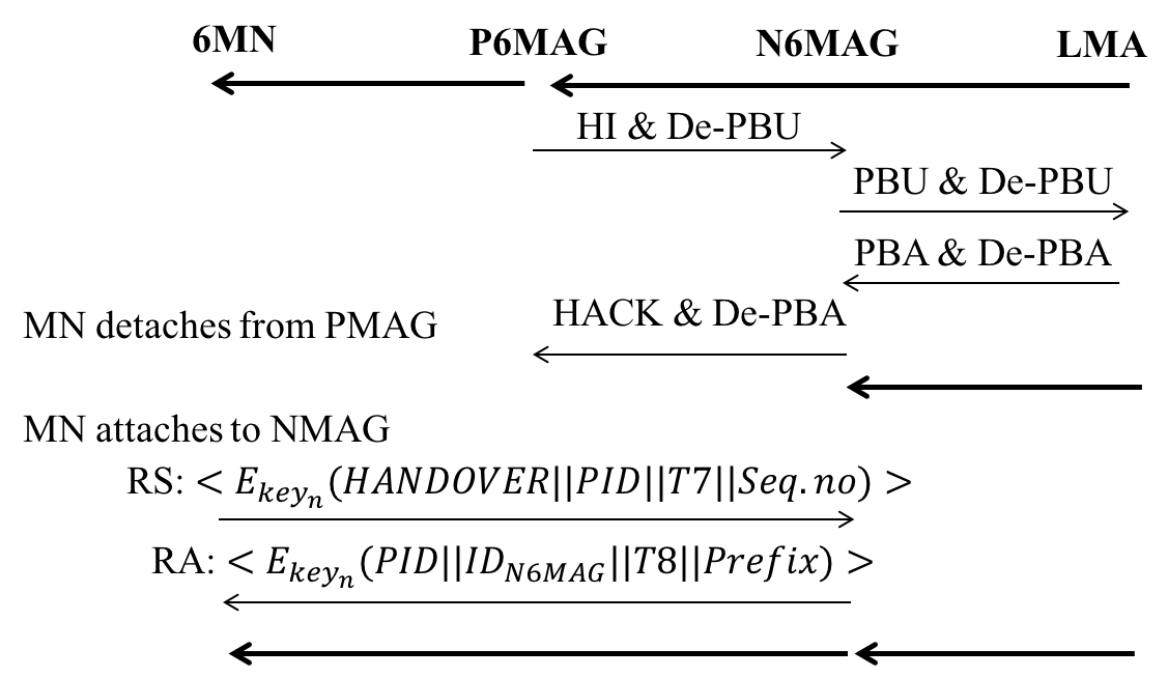

Figure 4.4 Handover Phase of ESPH

If a $6 \mathrm{MN}$ joins the 6LoWPAN network for the first time, it must follow the authentication steps as described in the initial AKE phase. When a $6 \mathrm{MN}$ is going to detach from the previous 6MAG (P6MAG), a HI and a de-registration PBU (De-PBU) message are sent to the target new 6MAG (N6MAG) predicted based on the direction of $6 \mathrm{MN}$ 's movement. The profile of this $6 \mathrm{MN}$ is sent within this request which is protected by the shared key $S K_{P 6 M A G \leftrightarrow L M A}$. In addition, the De-PBU message of the PMAG and the new PBU message are sent from the N6MAG to inform the LMA that the $6 \mathrm{MN}$ is going to roam in the domain of N6MAG. After checking the request, the LMA updates the binding cache entry for recording the N6MAG and sends the session $k e y_{n}$ in advance if this is the $n$th handover for the $6 \mathrm{MN}$. A bi-directional tunnel is built between the N6MAG and the LMA if the PBA message is valid. A HACK and a deregistration PBA (De-PBA) are sent to the P6MAG when N6MAG receives the acknowledge messages and the session key from the LMA. Thus, a bi-directional tunnel is established between these two 6MAGs before the detachment of the $6 \mathrm{MN}$, which is used for transferring data packets belonging to the $6 \mathrm{MN}$. Therefore, a marker message is added after the last data packet sent to the PMAG. The undelivered messages are transmitted to the N6MAG and the N6MAG starts to buffer the data packets when the P6MAG disconnects with the 6MN. 
When the $6 \mathrm{MN}$ attaches to the N6MAG, a RS message, which includes the encrypted handover information $E_{k e y_{n}}(H A N D O V E R\|P I D\| T 7 \| S e q . N o$ ), is sent to the N6MAG if the $6 \mathrm{MN}$ enters its domain. If the handover information can be successfully decrypted from the RS message, the correct sequence number is retrieved. Then, a RA message is sent to the $6 \mathrm{MN}$ in which the same network prefix is included. Thus, the $6 \mathrm{MN}$ can communicate with the N6MAG using the session key key $y_{n}$ to download the buffered data packets and successfully attaches to the N6MAG without changing its IP address.

The calculation of the key chain is performed according to the direction of the $6 \mathrm{MN}$ 's movement. It is assumed that the 6MAGs are uniformly distributed in the 6LoWPAN network and the coordinate of the $6 \mathrm{MAG}$, which the $6 \mathrm{MN}$ is initially attached to when entering the network, is $(x, y)$. The four initial values $\left(r t_{2}^{I D} \| y\right),\left(r t_{1}^{I D} \| y\right),\left(r t_{1} \| x\right),\left(r t_{2} \| x\right)$ - of the hash chains represent four geographical directions: north, south, east, and west, respectively. A hash chain $v$, $H_{1}(v), \ldots, H_{1}{ }^{i}(v), \ldots$ is generated from the root value $v$, where $i$ is the number of times that the $6 \mathrm{MN}$ is moving to a certain direction. The initial value of the key chain starts with the value $k e y_{0}=H_{1}\left(\left(r t_{1} \| x\right)\|0\|\left(r t_{2}^{I D} \| y\right) \| 0\right)$. The setting of the initial key $k e y_{0}$ is to confirm that the secret parameters $r t_{1}, r t_{2}, I D$ are correct. The rest of the key chain values are calculated according to the subsequent movement of the $6 \mathrm{MN}$. Suppose $v_{i}$ is the $i$ th value of the hash chain represented moving eastward or westward where $i$ is the number of times moving eastward or westward and $v_{j}$ is the $j$ th value of the hash chain represented moving northward or southward where $j$ is the number of times moving northward or southward. Thus, the key chain can be represented as $k e y_{n}=H_{1}\left(v_{i}\|i\| v_{j} \| j\right)$ where $n$ means the $n$th handover for the $6 \mathrm{MN}$. If a node moves, only the parts of the key material corresponding to the direction of the movement are updated while the rest of the key material remain the same as the previous key material. An example of the calculation of the key chain is depicted in Figure 4.5. The 6MN first attaches to the 6MAG whose coordinate is $(x, y)$ and starts communicating with this gateway using the initial key key. Suppose that this node is moving northward and 


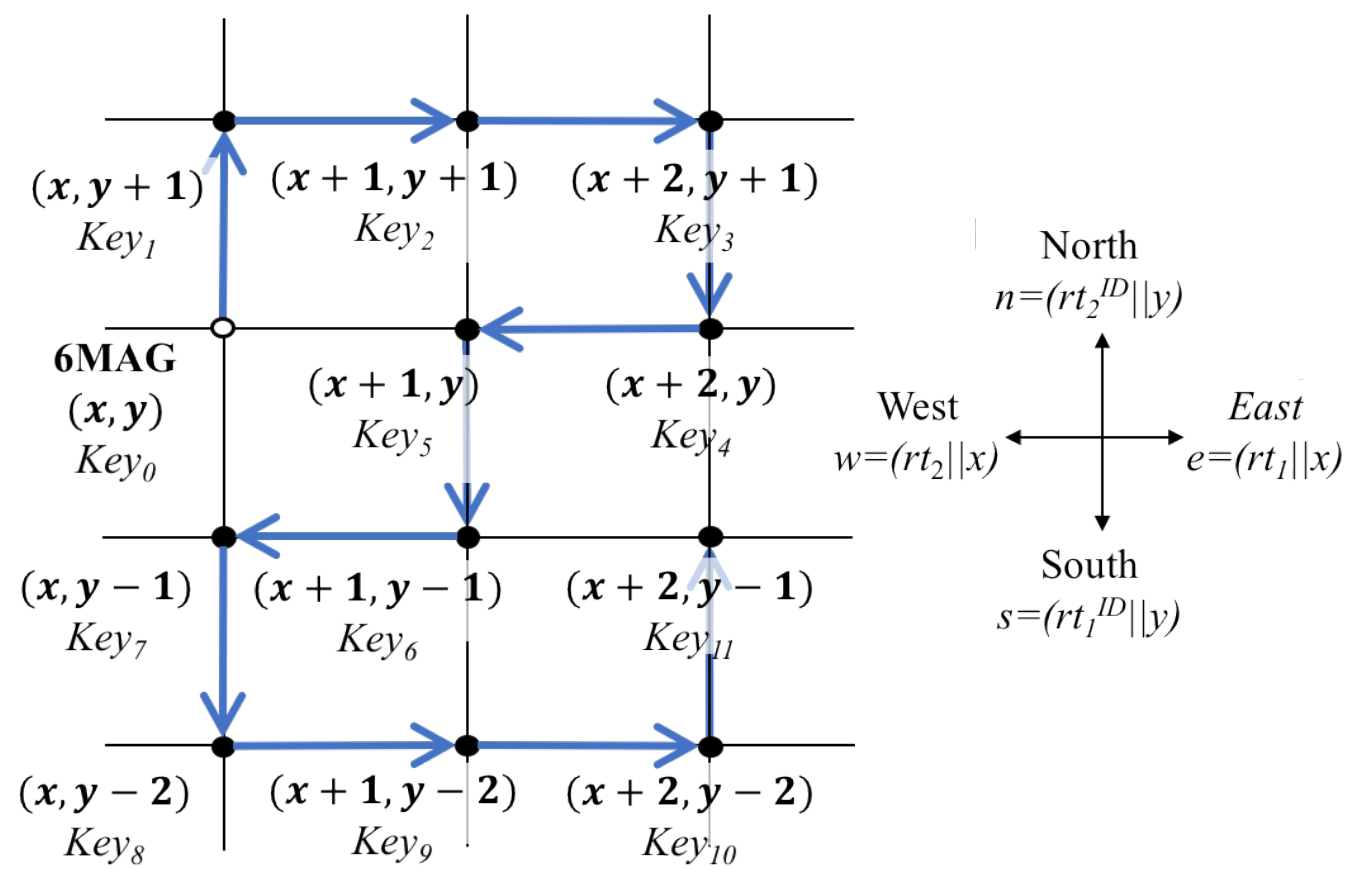

\begin{tabular}{|c|c|}
\hline 6MAG's Coordinate & Key \\
\hline$(\boldsymbol{x}, \boldsymbol{y})$ & $\mathrm{Key}_{0}=H_{1}(e\|0\| n \| 0)$ \\
\hline$(\boldsymbol{x}, \boldsymbol{y}+\mathbf{1})$ & $\mathrm{Key}_{1}=H_{1}\left(e\|0\| H_{1}(n) \| 1\right)$ \\
\hline$(\boldsymbol{x}+\mathbf{1}, \boldsymbol{y}+\mathbf{1})$ & $\mathrm{Key}_{2}=H_{1}\left(H_{1}(e)\|1\| H_{1}(n) \| 1\right)$ \\
\hline$(\boldsymbol{x}+\mathbf{2}, \boldsymbol{y}+\mathbf{1})$ & $\mathrm{Key}_{3}=H_{1}\left(H_{1}(e)^{2}\|2\| H_{1}(n) \| 1\right)$ \\
\hline$(\boldsymbol{x}+\mathbf{2}, \boldsymbol{y})$ & $\mathrm{Key}_{4}=H_{1}\left(H_{1}(e)^{2}\|2\| H_{1}(s) \| 1\right)$ \\
\hline$(\boldsymbol{x}+\mathbf{1}, \boldsymbol{y})$ & $\mathrm{Key}_{5}=H_{1}\left(H_{1}(w)\|1\| H_{1}(s) \| 1\right)$ \\
\hline$(\boldsymbol{x}+\mathbf{1}, \boldsymbol{y}-\mathbf{1})$ & $\mathrm{Key}_{6}=H_{1}\left(H_{1}(w)\|1\| H_{1}(s)^{2} \| 2\right)$ \\
\hline$(\boldsymbol{x}, \boldsymbol{y}-\mathbf{1})$ & $\mathrm{Key}_{7}=H_{1}\left(H_{1}(w)^{2}\|2\| H_{1}(s)^{2} \| 2\right)$ \\
\hline$(\boldsymbol{x}, \boldsymbol{y}-\mathbf{2})$ & $\mathrm{Key}_{8}=H_{1}\left(H_{1}(w)^{2}\|2\| H_{1}(s)^{3} \| 3\right)$ \\
\hline$(\boldsymbol{x}+\mathbf{1}, \boldsymbol{y}-\mathbf{2})$ & $\mathrm{Key}_{9}=H_{1}\left(H_{1}(e)^{3}\|3\| H_{1}(s)^{3} \| 3\right)$ \\
\hline$(\boldsymbol{x}+\mathbf{2}, \boldsymbol{y}-\mathbf{2})$ & $\mathrm{Key}_{10}=H_{1}\left(H_{1}(e)^{4}\|4\| H_{1}(s)^{3} \| 3\right)$ \\
\hline$(\boldsymbol{x}+\mathbf{2}, \boldsymbol{y}-\mathbf{1})$ & $\mathrm{Key}_{11}=H_{1}\left(H_{1}(e)^{4}\|4\| H_{1}(n)^{2} \| 2\right)$ \\
\hline
\end{tabular}

Figure 4.5 An Example of Key Chain Calculation 
attaches to a N6MAG, the session key is then changed to $k e y_{1}=H_{1}\left(\left(r t_{1} \| x\right) \|\right.$ $\left.0\left\|H_{1}\left(r t_{2}^{I D} \| y\right)\right\| 1\right)$ after the first handover. As the node begins to move eastward twice, the messages sent to the two $6 \mathrm{MAGs}$ by the $6 \mathrm{MN}$ are protected by $k e y_{2}=$ $H_{1}\left(H_{1}\left(r t_{1} \| x\right)\|1\| H_{1}\left(r t_{2}^{I D} \| y\right) \| 1\right)$ and $k^{2} y_{3}=H_{1}\left(H_{1}\left(r t_{1} \| x\right)^{2}\|2\| H_{1}\left(r t_{2}^{I D} \| y\right) \| 1\right)$ respectively. When the $6 \mathrm{MN}$ moves southward, the session key becomes key $_{4}=$ $H_{1}\left(H_{1}\left(r t_{1} \| x\right)^{2}\|2\| H_{1}\left(r t_{1}^{I D} \| y\right) \| 1\right)$ due to the first southward movement. The fifth handover, which is caused by the first westward movement, the $6 \mathrm{MN}$ communicates with the N6MAG using the key key $_{5}=H_{1}\left(H_{1}\left(r t_{2} \| x\right)\|1\| H_{1}\left(r t_{1}^{I D} \| y\right) \| 1\right)$. As the $6 \mathrm{MN}$ continuously moves following the path shown in Figure 4.5, the session key is $k e y_{10}=H_{1}\left(H_{1}\left(r t_{1} \| x\right)^{4}\|4\| H_{1}\left(r t_{1}^{I D} \| y\right)^{3} \| 3\right)$ after 10 handovers. The proposed session key chain generation approach can enable efficient handovers with fast authentication on the shared secret $r t_{1}, r t_{2}$ and the node's real ID which is known only to the node itself and the AAA server. Even if a current key is leaked, the attacker cannot obtain the previous or future keys which means that the proposed key chain calculation method achieves forward and backward secrecy. Although the location of the $6 \mathrm{MAG}$, which the $6 \mathrm{MN}$ initially attached to, is involved in the calculation of the key chain, this key generation method can prevent the same session key used when the $6 \mathrm{MN}$ moves in a domain again.

\subsection{Security Analysis}

In this section, the security of the proposed ESPH scheme is analyzed from three aspects. First, a proof of the logic correctness by using PCL will be provided to model the ESPH scheme and prove that the proposal achieves the mutual authentication and key agreement. Then, a formal verification is provided using AVISPA to verify the security functionalities. Finally, further analysis and explanation on the ability against various malicious attacks of the proposed solution are provided.

\subsubsection{Security Proof}

PCL [17-18] is a formal logic to prove the logic correctness and find vulnerabilities of a given network protocol. The consequences of individual protocol steps are 
provided by PCL with the consideration of the given sequence of actions and arbitrary actions of attackers. Existing methods based on model checking can only analyze bounded protocol executions while PCL provides an analysis with unbounded protocol executions. PCL can be used to prove security properties of network protocols without explicit reasoning about an intruder's actions while only reasoning about the actions of honest roles under unknown environmental conditions.

Before modelling the ESPH scheme by PCL, the parties involved in the operation of the protocol and the messages exchanged among them should be explicitly described in a simplified arrows-and-messages way as follows:

6MN $\rightarrow$ 6MAG: PID $\|T 1\| M s g \| u 1$

6MAG $\rightarrow$ LMA: PID $\left\|I D_{6 M A G}\right\| T 1\|T 2\| M s g \| u 2$

LMA $\rightarrow$ AAA: PID ||$D_{6 M A G}\left\|I D_{L M A}\right\| T 1\|T 3\| M s g \| u 3$

AAA $\rightarrow$ LMA: $P I D\|T 4\| u 4$

LMA $\rightarrow$ 6MAG: PID $\|T 5\| u 5$

$6 \mathrm{MAG} \rightarrow 6 \mathrm{MN}: E_{\text {key }}\left(P I D\left\|I D_{6 M A G}\right\| T 6 \|\right.$ Prefix $)$

1) Protocol Modelling

By the PCL, the first step of the security proof is the protocol modelling, which is to express the execution steps of all the participants, also called principals, in detail in order to formally state and prove the security properties of a protocol. By the ESPH, there are four participants including $6 \mathrm{MN}, 6 \mathrm{MAG}$, LMA and AAA server which are described as $\hat{X}, \hat{Y}, \hat{Z}$ and $\hat{S}$ respectively.

$$
\begin{aligned}
6 \mathrm{MN} \equiv(\hat{Y})[ \\
\\
\text { new } r_{1}, r_{2}, T 1 ; \\
S K_{6 M N \leftrightarrow A A A}:=\operatorname{HASH}(\operatorname{xor}(I D, R), P I D, T 1) ; \\
m 1:=\operatorname{symenc}\left(r_{1}, r_{2}, P W, \operatorname{HASH}\left(r_{1}, r_{2}\right)\right), S K_{6 M N \leftrightarrow A A A} ; \\
r t_{1}:=\operatorname{HASH}\left(P I D, r_{1}\right) ; \\
r t_{2}:=\operatorname{HASH}\left(P I D, r_{2}\right) ; \\
k e y_{n}:=\operatorname{HASH}\left(r t_{1}, r t_{2}, m, n\right) ; \\
\\
M A C 1:=\operatorname{HMAC}\left(k_{r},(P I D, T 1, m 1) ;\right.
\end{aligned}
$$


$U 1:=\operatorname{xor}(\operatorname{sig}, M A C 1)$;

Send $\hat{X}, \hat{Y},(P I D, T 1, m 1, U 1)$;

Receive $\hat{Y}, \hat{X}, m 2$;

PID $, I D_{6 M A G}, T 6$, Prefix:= symdec $m 2$, key $_{0}$;

]X

$6 \mathrm{MAG} \equiv(\hat{X}, \hat{Z})[$

Receive $\widehat{X}, \hat{Y},(P I D, T 1, m 1, U 1)$;

Check T1;

$M A C 1^{\prime}:=\operatorname{HMAC}(k e y,(P I D, T 1, m 1)$;

Verify $\operatorname{xor}\left(U 1, M A C 1^{\prime}\right),\left(P I D, I D_{A A A}\right), P u b_{A A A}$;

new $T 2$;

$M A C 2:=\operatorname{HMAC}\left(S K_{6 M A G \leftrightarrow L M A},\left(P I D, I D_{6 M A G}, T 1, T 2, m 1\right)\right.$;

$U 2:=\operatorname{xor}(\operatorname{sig}, M A C 2)$;

Send $\widehat{Y}, \hat{Z},\left(P I D, I D_{6 M A G}, T 1, T 2, m 1, U 2\right)$;

Receive $\hat{Z}, \hat{Y},(P I D, T 5, U 5)$;

Check T5;

$M A C 5^{\prime}:=\operatorname{HMAC}\left(S K_{6 M A G \leftrightarrow L M A},(P I D, T 5)\right.$;

key ${ }_{0}{ }^{\prime}, H A S H\left(k e y_{0}\right)^{\prime}:=\operatorname{xor}\left(U 5, M A C 5^{\prime}\right)$;

Match $\operatorname{HASH}\left(k e y_{0}{ }^{\prime}\right) / \operatorname{HASH}\left(k e y_{0}\right)^{\prime}$;

new T6;

$m 2:=\operatorname{symenc}\left(P I D, I D_{6 M A G}, T 6\right.$, Prefix $)$, key $y_{0} ;$

Send $\hat{Y}, \hat{X}, m 2$;

\rceil$\widehat{Y}$

$\operatorname{LMA} \equiv(\hat{Y}, \hat{S})[$

Receive $\hat{Y}, \hat{Z},\left(P I D, I D_{6 M A G}, T 1, T 2, m 1, U 2\right)$;

Check T2;

$M A C 2^{\prime}:=\operatorname{HMAC}\left(S K_{6 M A G \leftrightarrow L M A},\left(P I D, I D_{6 M A G}, T 1, T 2, m 1\right) ;\right.$ 
Verify $\operatorname{xor}\left(U 2, M A C 2^{\prime}\right),\left(P I D, I D_{A A A}\right), K p u b_{A A A}$;

new T3;

$M A C 3:=\operatorname{HMAC}\left(S K_{L M A \leftrightarrow A A A},\left(P I D, I D_{6 M A G}, I D_{L M A}, T 1, T 3, m 1\right)\right.$;

$U 3:=\operatorname{xor}(\operatorname{sig}, M A C 3)$;

Send $\hat{Z}, \hat{S},\left(P I D, I D_{6 M A G}, I D_{L M A}, T 1, T 3, m 1, U 3\right)$;

Receive $\hat{S}, \hat{Z},(P I D, T 4, U 4)$;

Check T4;

$M A C 4^{\prime}:=H M A C\left(S K_{L M A \leftrightarrow A A A},(P I D, T 4)\right.$;

$r t_{1}{ }^{\prime}, r t_{2}{ }^{\prime}, r t_{1}^{I D}{ }^{\prime}, r t_{2}^{I D}{ }^{\prime}, \operatorname{HASH}\left(r t_{1}, r t_{2}, r t_{1}^{I D}, r t_{2}^{I D}\right):=\operatorname{xor}\left(U 4, M A C 4^{\prime}\right)$;

Match HASH $\left(r t_{1}{ }^{\prime}, r t_{2}{ }^{\prime}, r t_{1}^{I D}{ }^{\prime}, r t_{2}^{I D}{ }^{\prime}\right) / \operatorname{HASH}\left(r t_{1}, r t_{2}, r t_{1}^{I D}, r t_{2}^{I D}\right)^{\prime}$;

new T5;

key $_{n}:=\operatorname{HASH}\left(r t_{1}, r t_{2}, m, n\right)$;

$M A C 5:=\operatorname{HMAC}\left(S K_{6 M A G \leftrightarrow L M A},(P I D, T 5)\right.$;

$U 4:=\operatorname{xor}\left(\left(\right.\right.$ key $_{0}, \operatorname{HASH}\left(\right.$ key $\left.\left._{0}\right)\right)$, MAC5);

Send $\hat{Z}, \hat{Y},(P I D, T 5, U 5)$;

]$\hat{Z}$

\section{$\mathrm{AAA} \equiv(\hat{Z})[$}

Receive $\hat{Z}, \hat{S}$, $\left(P I D, I D_{6 M A G}, I D_{L M A}, T 1, T 3, m 1, U 3\right)$;

Check T3;

$M A C 3^{\prime}:=\operatorname{HMAC}\left(S K_{L M A \leftrightarrow A A A},\left(P I D, I D_{6 M A G}, I D_{L M A}, T 1, T 3, m 1\right)\right.$;

Verify xor(U3,MAC3'), (PID, ID $\left.D_{A A A}\right), K p u b_{A A A}$;

$S K_{6 M N \leftrightarrow A A A}:=\operatorname{HASH}(\operatorname{xor}(I D, R), P I D, T 1)$

$m 1:=\operatorname{symdec}\left(r_{1}, r_{2}, P W, \operatorname{HASH}\left(r_{1}, r_{2}\right)\right), S K_{6 M N \leftrightarrow A A A} ;$

$\operatorname{Match} \operatorname{HASH}\left(r_{1}{ }^{\prime}, r_{2}{ }^{\prime}\right) / \operatorname{HASH}\left(\left(r_{1}, r_{2}\right)^{\prime}\right.$;

$r t_{1}:=\operatorname{HASH}\left(P I D, r_{1}\right)$;

$r t_{2}:=\operatorname{HASH}\left(P I D, r_{2}\right)$;

new T4;

$M A C 4:=\operatorname{HMAC}\left(S K_{L M A \leftrightarrow A A A},(P I D, T 4) ;\right.$ 


$$
U 4:=\operatorname{xor}\left(\left(r t_{1}, r t_{2}, r t_{1}^{I D}, r t_{2}^{I D}, \operatorname{HASH}\left(r t_{1}, r t_{2}, r t_{1}^{I D}, r t_{2}^{I D}\right)\right), M A C 4\right)
$$

Send $\hat{S}, \hat{Z},(P I D, T 4, U 4)$;

]$\widehat{S}$

2) Precondition and Invariants

By the PCL, in the second stage of the security proof, the preconditions of a protocol should be stated and established. With the satisfaction of the preconditions, the protocol can be ensured to execute correctly. For example, for initiator $\hat{X}$, the precondition of the ESPH is represented as $\theta_{E S P H}$ that the $6 \mathrm{MN}$ and the server have the shared secrets before the $6 \mathrm{MN}$ is employed in the 6LoWPAN network.

$$
\begin{aligned}
& \theta_{E S P H}=(\operatorname{Honest}(\hat{X}) \wedge \operatorname{Honest}(\hat{S})) \\
& \supset\left(\operatorname{Has}\left(\hat{X},\left(I D, P W, P I D, k_{r}, r_{0}, \sigma\right)\right) \wedge \operatorname{Has}\left(\hat{S},\left(I D, P I D, \operatorname{HASH}\left(I D, P W, r_{0}\right), r_{0}, \sigma\right)\right)\right)
\end{aligned}
$$

The invariants, which characterize the environment where the protocol can operate and preserve its security properties, should be stated to proceed with the security proof. By the PCL, the invariants can be proved by the honesty rule that if a property holds before each role starts, and is preserved after any sequence of actions performed by an honest principal, then the property holds for every honest principal [67]. Invariant $\Gamma_{E S P H}$ is described as follows.

$$
\begin{aligned}
& \Gamma_{E S P H}=\operatorname{Send}(Z, t 1) \wedge \text { Contains }\left(t 1,\left(r_{1}, r_{2}\right)\right) \\
& \supset\left(\operatorname { N e w } ( Z , ( r _ { 1 } , r _ { 2 } ) ) \vee \left(\operatorname{Receive}(Z, t 2) \wedge \text { Contains }\left(t 2,\left(r_{1}, r_{2}\right)\right)\right.\right. \\
& <\operatorname{Send}(Y,(\hat{Y}, \hat{X}, m 2)) \\
& \text { 3) } \operatorname{Security} \text { Property }
\end{aligned}
$$

By the PCL, in the final stage of the security proof, the security properties of the protocol should be demonstrated that each sending action takes place before the corresponding receiving action. Each message sent by the initiator should be received by the responder and vice versa. Moreover, the order of sending or receiving actions executed by each principal should be the same. To prove the security property of the proposed protocol, some axioms and rules [67, 68] have been used summarized as follows.
AA1. $\mathrm{T}[a]_{X} \mathrm{a}$ 
Axiom AA1 states that if the precondition is true, after an action has been executed by a principal, the corresponding predicate which asserts that the action happened in the past is true.

AA4. $\mathrm{T}[a ; \ldots ; b]_{X} \mathrm{a}<\mathrm{b}$

Axiom AA4 states that after actions $a, \cdots, b$ have been done by the thread $X$ sequentially, the action predicates that the actions happened in order.

AN1. $\operatorname{New}(X, x) \wedge \operatorname{New}(Y, x) \supset X=Y$

Axiom AN1 states that a nonce is created only by a unique thread.

AN3. $T[\text { new } x]_{X} \operatorname{Fresh}(X, x)$

Axiom AN3 states that a new generated nonce $x$ is fresh.

REC. Receive $(X, x) \supset \operatorname{Has}(X, x)$

REC states that if a principal receives the term $x$, then the principal has it.

PROJ. $\operatorname{Has}(X,(x, y)) \supset \operatorname{Has}(X, x) \wedge \operatorname{Has}(X, y)$

PROJ allows a tuple to decompose itself into its components

DEC. $\operatorname{Has}\left(X, E N C_{K}\{|x|\}\right) \wedge \operatorname{Has}(X, K) \supset \operatorname{Has}(X, x)$

DEC allows an encrypted term can be decrypted if the corresponding secret key is known.

SEQ. $\frac{\phi_{1}[P]_{X} \phi_{2} \quad \phi_{2}\left[P^{\prime}\right]_{X} \phi_{3}}{\phi_{1}\left[P P^{\prime}\right]_{X} \phi_{3}}$

Sequencing rule states that the two cords $P$ and $P^{\prime}$ can be sequentially composed when the post-condition of $P$ and the pre-condition of $P^{\prime}$ are match.

P1. Persist $(X, t)[a]_{X}$ Persist $(X, t)$ for Persist $\in\{$ Has, FirstSend, a, Gen $\}$

Axiom P1 states that if certain predicates, such as Has, FirstSend, a, Gen, are true, they will be held after further actions.

FS1. Fresh $(X, t)\left[\text { send } t^{\prime}\right]_{X}$ FirstSend $\left(X, t, t^{\prime}\right)$, where $t \subseteq t^{\prime}$

Axioms FS1 states that if a fresh nonce $t$ is generated by a thread $X$ and will be sent in message $t^{\prime}$, it is the first send event of nonce $t$ after the execution of the action sending $t^{\prime}$ by the thread $X$.

FS2. FirstSend $\left(X, t, t^{\prime}\right) \wedge a\left(Y, t^{\prime \prime}\right) \supset \operatorname{Send}\left(X, t^{\prime}\right)<\mathrm{a}\left(Y, t^{\prime \prime}\right)$, where $X \neq Y$ and $t \subseteq t^{\prime \prime}$ 
Axiom FS2 states that if a thread $Y$ does some action with the term $t$ " which contains the term $t$ and the thread $X$ sent inside the term $t^{\prime}$ before, then the sending action by $X$ must have happened before $Y$ 's action.

HON. $\frac{\operatorname{Start}(X)[]_{X} \phi \quad \forall \rho \in Q . \forall P \in B S(\rho) . \phi[P]_{X} \phi}{\operatorname{Honest}(\widehat{X}) \supset \phi}$

The honesty rule $\mathrm{HON}$ is applied for proving that certain actions of other parties in the protocol have been performed or not.

HASH4. Has $\left(X, \operatorname{HASH}_{K}(x)\right) \supset \operatorname{Computes}\left(Y, \operatorname{HASH}_{K}(x)\right) \vee \exists Y, m$. Computes( $\left.Y, \operatorname{HASH}_{K}(x)\right) \wedge \operatorname{Send}(Y, m) \wedge \operatorname{Contains}\left(m, \operatorname{HASH}_{K}(x)\right)$

HASH4 states that if thread $X$ has a hash value, the hash value is computed by $X$ itself or others.

- Security Property of the $6 \mathrm{MN}$

The formal property of $6 \mathrm{MN}_{\mathrm{ESPH}}$ is described as follows:

$$
\Gamma_{E S P H} \rightarrow \theta_{E S P H}\left[6 \mathrm{MN}_{\mathrm{ESPH}}\right]_{X} \operatorname{Honest}(\hat{Y}) \wedge \hat{Y} \neq \hat{X} \supset \phi_{6 M N}
$$

The formula $\theta[P]_{X} \phi$ means that if the precondition $\theta$ is true, the postcondition $\phi$ is true after $X$ executes actions $P$. The postcondition $\phi_{6 L H}$ means that if $\hat{Y}$ is assumed to be honest, $\hat{X}$ is guaranteed that $\hat{Y}$ participates in ESPH and each sending and receiving action happens in order after the actions executed by $6 \mathrm{MN}_{\mathrm{ESPH}}$.

$$
\begin{aligned}
\phi_{6 M N} \equiv & \exists Y .((\operatorname{Send}(X, m s g 1)<\operatorname{Receive}(Y, m s g 1)) \\
& <(\operatorname{Receive}(Y, m s g 1)<\operatorname{Send}(Y, m s g 2)) \\
& <(\operatorname{Send}(Y, m s g 2)<\operatorname{Receive}(X, m s g 2))),
\end{aligned}
$$

where $m s g 1$ and $m s g 2$ are $(\widehat{X}, \widehat{Y},(P I D, T 1, m 1, U 1))$ and $(\widehat{Y}, \widehat{X}, m 2)$ respectively.

Proof. A proof of the security property is shown as follows:

(1) AN3 $\theta_{E S P H}\left[\text { new } r_{1}, r_{2}\right]_{X} \operatorname{Fresh}\left(X,\left(r_{1}, r_{2}\right)\right)$

(2) FS1 $\operatorname{Fresh}\left(X,\left(r_{1}, r_{2}\right)\right)[\operatorname{send} \hat{X}, \hat{Y},(P I D, T 1, m 1, U 1)]_{X}$ FirstSend $(X$,

$$
\left.\left(r_{1}, r_{2}\right),(\hat{X}, \hat{Y},(P I D, T 1, m 1, U 1))\right)
$$

(3) (1), (2), SEQ, P1

$$
\theta_{E S P H}\left[6 \mathrm{MN}_{\mathrm{ESPH}}\right]_{X} \operatorname{FirstSend}\left(X,\left(r_{1}, r_{2}\right),(\hat{X}, \widehat{Y},(P I D, T 1, m 1, U 1))\right)
$$

(4) (3), FS2 $\theta_{E S P H}\left[6 \mathrm{MN}_{\mathrm{ESPH}}\right]_{X} \operatorname{Receive}(Y,(\widehat{X}, \widehat{Y},(P I D, T 1, m 1, U 1))) \wedge \hat{Y} \neq \hat{X}$

$$
\supset \operatorname{Send}(X,(\hat{X}, \widehat{Y},(P I D, T 1, m 1, U 1)))
$$




$$
<\operatorname{Receive}(Y,(\widehat{X}, \widehat{Y},(P I D, T 1, m 1, U 1)))
$$

(5) AA1, P1, SEQ, DEC

$$
\begin{aligned}
& \theta_{E S P H}\left[6 \mathrm{MN}_{\mathrm{ESPH}}\right]_{X} \operatorname{Receive}(X,(\hat{Y}, \hat{X}, m 2)) \wedge \operatorname{Has}\left(X, \text { key }_{0}\right) \\
& \supset \operatorname{Has}(X, T 6) \supset \operatorname{Has}\left(Y, \text { key }_{0}\right)
\end{aligned}
$$

(6) (5), HASH4

$$
\theta_{E S P H}\left[6 \mathrm{MN}_{\mathrm{ESPH}}\right]_{X} \exists Z, t 1 . \operatorname{Send}(Z, t 1) \wedge \text { Contains }\left(t 1,\left(r_{1}, r_{2}\right)\right)
$$

(7) $\operatorname{HON}\left(\operatorname{Honest}(\hat{Y}) \wedge \operatorname{Has}\left(Y, k e y_{0}\right)\right.$

$$
\begin{aligned}
& \supset\left(\operatorname { N e w } ( Y , \text { key } _ { 0 } ) \vee \left(\operatorname{Receive}(Z, t 1) \wedge \operatorname{Contains}\left(t 1,\left(r_{1}, r_{2}\right)\right)\right.\right. \\
& <\operatorname{Send}(Y,(\hat{Y}, \hat{X}, m 2))))
\end{aligned}
$$

(8) (1), (7), SEQ, AN1

$$
\begin{aligned}
& \theta_{E S P H}\left[\operatorname{MMN}_{\mathrm{ESPH}}\right]_{X} \operatorname{Honest}(\hat{Y}) \wedge \hat{Y} \neq \hat{X} \\
& \supset(\exists Y . \text { Receive }(Y,(\hat{X}, \hat{Y},(P I D, T 1, m 1, U 1)))<\operatorname{Send}(Y,(\hat{Y}, \hat{X}, m 2))
\end{aligned}
$$

(9) (5), (6), (8), SEQ

$$
\begin{aligned}
& \theta_{E S P H}\left[6 \mathrm{MN}_{\mathrm{ESPH}}\right]_{X} \operatorname{Honest}(\hat{Y}) \wedge \hat{Y} \neq \hat{X} \wedge \hat{Y} \neq \hat{Z} \\
\supset & \exists Z, t 1 . \operatorname{Send}(Z, t 1) \wedge \operatorname{Contains}\left(t 1,\left(r_{1}, r_{2}\right)\right) \\
< & \operatorname{Has}(Y, m 2))<\operatorname{Receive}(X,(\widehat{Y}, \widehat{X}, m 2)) \\
\supset & \operatorname{Send}(Y,(\hat{Y}, \hat{X}, m 2))<\operatorname{Receive}(X,(\widehat{Y}, \hat{X}, m 2))
\end{aligned}
$$

$$
\text { (4), (8), (9) } \theta_{E S P H}\left[6 \mathrm{MN}_{\mathrm{ESPH}}\right]_{X} \operatorname{Honest}(\hat{Y}) \wedge \hat{Y} \neq \hat{X} \supset \phi_{6 M N}
$$

By now, each sending and receiving event of the principal $\widehat{X}, \hat{Y}$ have been proved to happen in order. Here, the authentication property for $\widehat{X}$ is only presented. The proof for $\hat{Y}, \hat{Z}$ and $\hat{S}$, which can be derived in the similar way, are omitted.

\subsubsection{Formal Verification}

AVISPA is a formal verification tool which can automatically validate the network security protocols and applications. Two back-ends, the CL-Atse and the OFMC are employed in the security analysis of ESPH.

As HLPSL is a role-based language, there are four basic roles in the ESPH scheme by the HLPSL code, which are the $6 \mathrm{MN}$, the $6 \mathrm{MAG}$, the LMA, the AAA server. Actions of each role in the ESPH protocol are specified in the basic role module. A 
composed role "session" instantiates the four basic roles so that they can execute in parallel. A top-level role "environment", which contains global constants and a composition of sessions, is defined. In addition, the initial knowledge that an intruder has is specified in this module. The intruder model is defined in section 4.2.3. It is assumed that the intruder to the proposed protocol initially has the identities and public keys of all the participants in the proposed protocol, its own private key, symmetric keys shared with other entities, and other public functions. Finally, the security goals are defined, by which the keys are kept secret among the corresponding roles and the roles can be mutually authenticated. The initial authentication and the handover phases are coded in HLPSL and are analyzed by the two above-mentioned back-ends. The output results of ESPH using these two back-ends, as shown in Figure 4.6, demonstrate that the protocol is safe under the threat model. In CL-AtSe, 5464 states are analyzed and 324 states are reachable. It takes $0.65 \mathrm{~s}$ for protocol translation and $135.02 \mathrm{~s}$ for algebraic computation. In OFMC, the protocol is analyzed with limited depth (the depth for the search is 9). The total number of nodes searched is 5768 , which takes $29.92 \mathrm{~s}$.

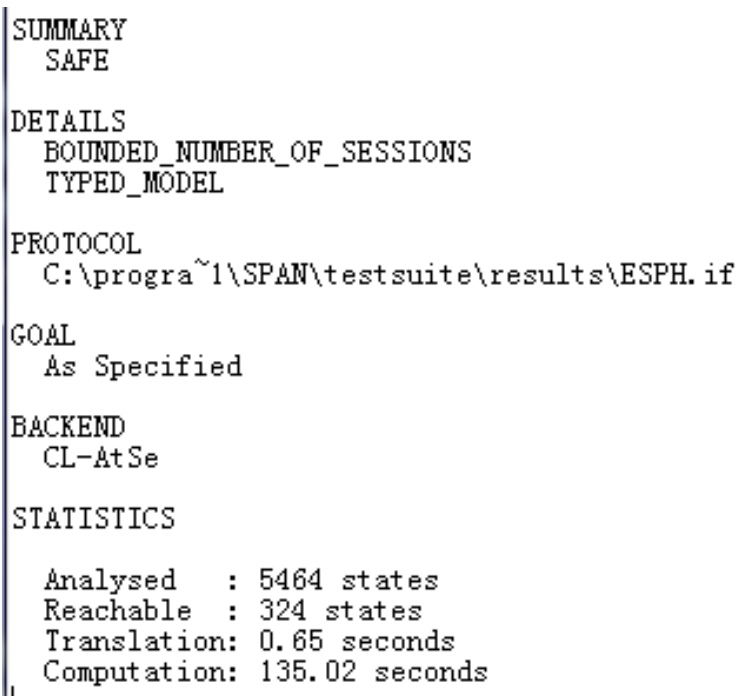

a) Output Results using CL-Atse 


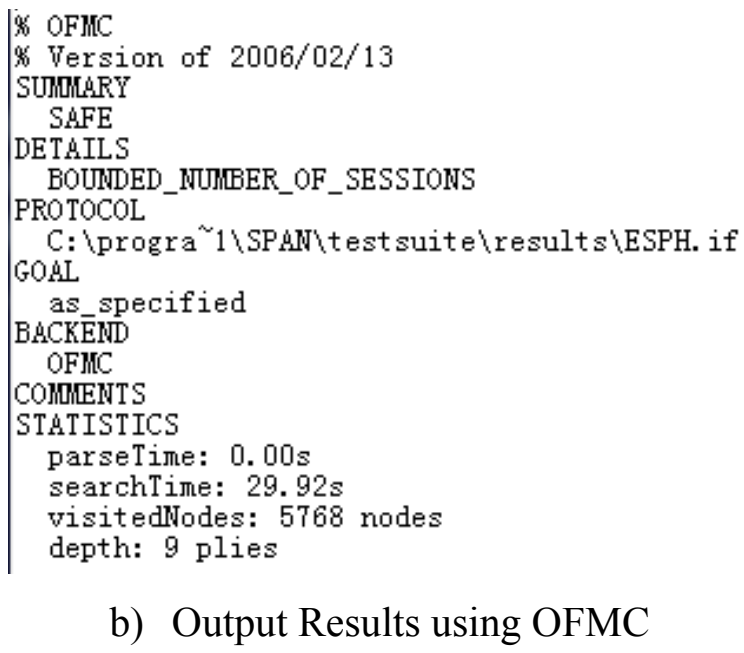

b) Output Results using OFMC

Figure 4.6 Output Results of ESPH using AVISPA

\subsubsection{Ability against Malicious Attacks}

1) Replay Attacks

A replay attack is an action to send the previously obtained messages to a receiver to prove that the message is valid and sent from a legitimate device. Thus, the attacker can extract confidential information from the response message. By SPAM, although nonce can be used to prevent the replay attacks, the receiver cannot distinguish whether the nonce contained in the first authentication message is fresh or not. By the ESPH scheme, if one of the transmitted messages is obtained by the attacker and resent to the receiver, the receiver can compare the timestamp in the message with the receiving time. If the time difference is greater than a predefined threshold, the message will be considered as invalid to be ignored. Even if the attacker can alter or delete the timestamp, this attack cannot succeed since the message is protected with a MAC ${ }^{2}$ value.

2) MITM Attacks

By an MITM attack, an adversary can eavesdrop, alter or delete the transmitted messages between two parties to make them believe that they directly communicate with each other. By SPAM, since a group key PSK is shared among the MAGs and the LMA, any MAG can derive the message encrypted by the PSK and re-encrypt. By the 
ESPH scheme, since the MAC ${ }^{2}$ and the signature have been employed for the protection, the MITM attacks can be prevented as well. For example, although a transmitted message $<P I D, I D_{6 M A G}, T 2, M s g, \sigma \oplus M A C 2>$ can be obtained by the attacker, the signature value cannot be successfully obtained because the attacker does not have the session key. Besides, the attacker is unable to decrypt Msg to retrieve the secret value $r_{1}$ and $r_{2}$ without the session key $S K_{6 M N \leftrightarrow A A A}$.

3) Impersonation Attacks

An impersonation attack can be launched by an adversary disguising as a legitimate party in the system. By SPAM, although the MN is registered with the AAA server beforehand, the AAA server is not involved in the initial authentication and handover phase. Thus, a dishonest MAG can impersonate many MNs which may not exist. By the ESPH scheme, since all of the devices are assumed to have registered with the server before the deployment, if an adversary impersonates a $6 \mathrm{MN}$, the server will check its legitimacy by comparing the secret values included in the messages with the ones stored in its database. When a legitimate $6 \mathrm{MN}$ sleeps, an attacker may take its legal IP address to launch malicious attacks. However, it cannot success without knowing the session key $k e y_{n}$. Thus, the attacker cannot successfully impersonate a legal device without having the corresponding shared secrets.

4) Privileged Insider Attacks

An insider attack is launched by an authorized and legal entity that has access to the confidential information of its organization to steal the security data or inject fraudulent data to the system. By SPAM, some secret parameters assigned to each MN are the same. Thus, a dishonest MAG can retrieve the real ID of an MN and obtain every message sent from the MN. By the ESPH scheme, since the password is only known to the $6 \mathrm{MN}$ without leaking to other parties, the insider is not able to retrieve the password. Besides, the real identity registered with the server, which is assumed trustworthy, cannot be sent to other 6MAGs or LMA. Moreover, the root values used for generating the session key are protected by the symmetric key between the $6 \mathrm{MN}$ and the server. Therefore, the 6MN's information cannot be extracted by the insider of the network. 


\section{5) Sybil Attacks}

By a Sybil attack, an attacker can forge multiple identities to cheat the 6MAGs or the LMA that a large number of legal devices connect or communicate with them, which could consume too much network resources. SPAM is vulnerable to this kind of attacks. If the MAG is compromised or there is a dishonest MAG, the adversary can disguise as many MNs using secret parameters. Other devices cannot distinguish whether the $\mathrm{MN}$ is legal or not because the AAA server is not involved in the authentication and handover phase. The Sybil attacks can be prevented by the ESPH scheme because a $6 \mathrm{MN}$ must be registered with the server before the deployment. Thus, the $6 \mathrm{MNs}$ with fake identities and security parameters cannot pass the initial authentication phase with the AAA server. Table 4.2 lists the comparison of security properties between ESPH scheme and the SPAM scheme.

Table 4.2 Comparisons of Security Properties between ESPH and SPAM

\begin{tabular}{ccc}
\hline \hline Properties & ESPH & SPAM \\
\hline Type of cryptosystem & Hybrid & Symmetric \\
Support for mobility & Yes & Yes \\
Against replay attack & Yes & No \\
Against MITM attack & Yes & No \\
Against impersonation attack & Yes & No \\
Against Sybil attack & Yes & No \\
Output result of AVISPA & SAFE & UNSAFE \\
\hline \hline
\end{tabular}

\subsection{Performance Evaluation}

In this section, the performance of the ESPH scheme is compared with the SPAM in term of computational overhead. The cryptographic algorithms applied in the 
simulation are SHA-256, Hash-based Message Authentication Code (HMAC)-SHA256, AES-Cipher Block Chaining (CBC)-256 and the ECDSA [81] with prime192v1. Suppose that the length of the message is 1024 bits, the time consumption of each cryptographic operation is analyzed by JAVA and conducted on Intel(R) Core(TM) i52400 CPU@3.10 GHz, 4 GB RAM, Windows 7. The average computational overhead of each operation and the variances computed through 1000 times of calculation are listed in Table 4.3.

Table 4.3 Computational Time of Cryptographic Operations

\begin{tabular}{cc}
\hline \hline Operations & Time $(\mathrm{ms})$ \\
\hline SHA-256 & $0.0601 \quad(0.0021)$ \\
HMAC-SHA256 & $0.0789(0.00059)$ \\
AES-CBC-256 & $0.1447 \quad(0.0028)$ \\
ECDSA Signing & $0.8521 \quad(0.0389)$ \\
ECDSA Verification & $0.9060 \quad(0.0420)$ \\
\hline \hline
\end{tabular}

Although SPAM uses simple hash function and symmetric encryption which causes less time consumption, it has some security problems that need to be solved. To provide more secure functionalities to the 6LoWPAN network, ESPH employs more complicated algorithms for the 6MAGs, the LMA and the server which have more capabilities to perform ECDSA. Thus, the computational overhead of the initial authentication of the ESPH scheme is higher than that of the SPAM scheme. The time consumption in the initial authentication phase of the SPAM scheme and the ESPH scheme are $2.9629 \mathrm{~ms}$ and $5.3858 \mathrm{~ms}$, respectively. Although the time consumption is higher than that of the SPAM scheme due to employing the ECDSA method, the computational overheads at the $6 \mathrm{MNs}$ by the SPAM scheme and by the ESPH scheme are $0.7346 \mathrm{~ms}$ and $0.5486 \mathrm{~ms}$, respectively. The more complicated calculations have been migrated to the components with more resources such as the 6MAGs, the LMA 
and the AAA server. Therefore, ESPH can achieve a better performance at the resource constrained devices.

Though the attacks as claimed previously can be prevented, but other types of malicious attacks as well as some unforeseen attacks may cause the interruption of the execution of the protocol during the initial authentication phase. So, it is assumed that an interruption may randomly occur at any step of the protocol execution. The protocol cannot continue if an attack successfully breaks it in the execution. With the increasing number of successful attacks, the average total time delay for a complete execution of the protocol will take longer. The comparison of the total time delay of the $6 \mathrm{MN}$ between ESPH and SPAM is shown in Figure 4.7 (a). The number of attacks used in the simulation is set to 10000 . It is shown that the ESPH scheme has less computational overhead of the $6 \mathrm{MN}$ in the initial authentication phase than that of the SPAM scheme.

To support seamless handovers of the $6 \mathrm{MNs}$, a key chain has been used in the handover phase so that a $6 \mathrm{MN}$ will not need to perform a full authentication procedure with the AAA server each time when it roams into the area of a new N6MAG. However, a full authentication procedure may still be required for safety which depends on the requirements of a specific system. By the ESPH scheme, the initial authentication takes a longer period of time due to the computation of the ECDSA and the generation of the key chain. However, the computational overhead of each handover by the SPAM and by the ESPH are $3.8912 \mathrm{~ms}$ and $1.7965 \mathrm{~ms}$, respectively. In order to demonstrate that the ESPH can achieve a better performance even with higher computational overhead in the initial authentication phase, the average time consumption in the handover phase is defined using the equation $T_{A H}=\frac{T_{I A}+T_{H} \times x}{x}$, where $T_{A H}$ represents the average time consumption of the handover phases, $T_{I A}$ represents the time consumption of the initial authentication phase, $T_{H}$ represents the time consumption of each handover and $x$ is the number of handovers. Therefore, based on the above definitions, the comparison of the computational overheads between these two schemes can be depicted in Figure 4.7 (b) and the detailed data is 
presented in Table 4.4, which shows that with the increasing number of 6MAGs to which the MN has attached, the computational overhead caused by the ESPH scheme is less than that by the SPAM scheme after 2 handovers.

Table 4.4 Comparison of Average Time Consumption between ESPH and SPAM

\begin{tabular}{ccc}
\hline \hline Number of Handovers & SPAM (ms) & ESPH (ms) \\
\hline 1 & 2.9629 & 5.3858 \\
2 & 6.8541 & 7.1823 \\
3 & 5.3726 & 4.4894 \\
4 & 4.8788 & 3.5918 \\
5 & 4.6319 & 3.1429 \\
\hline \hline
\end{tabular}

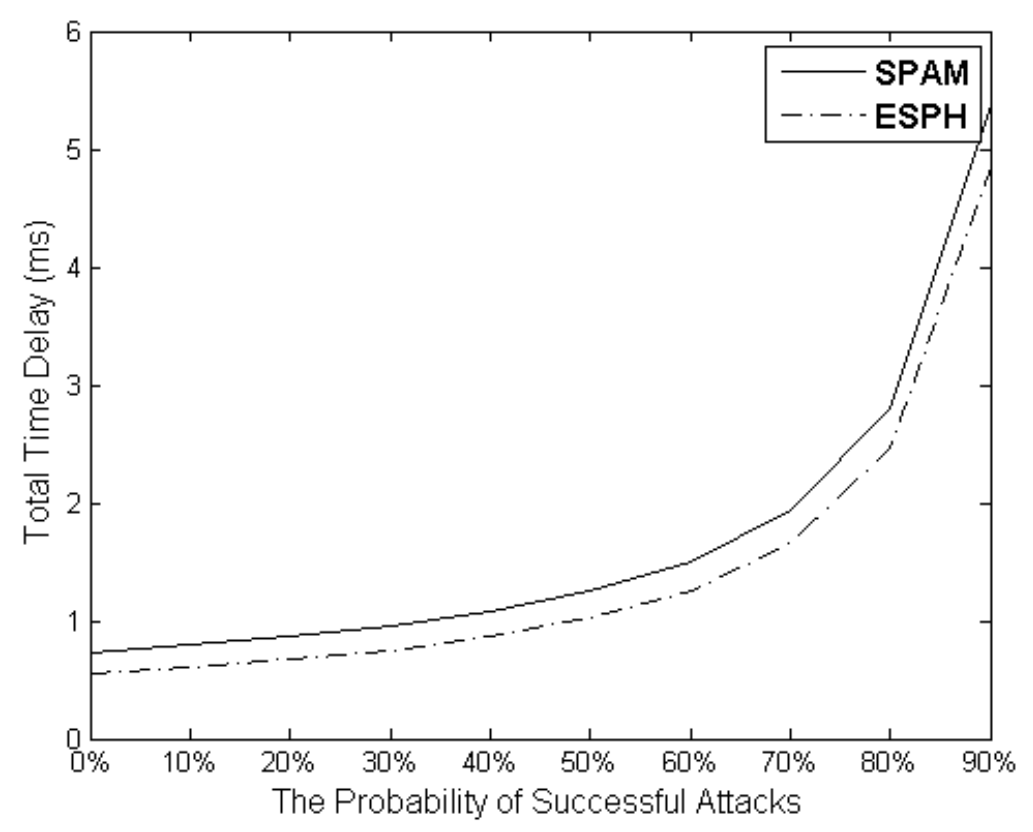

(a) Time Consumption in the Initial Authentication Phase 


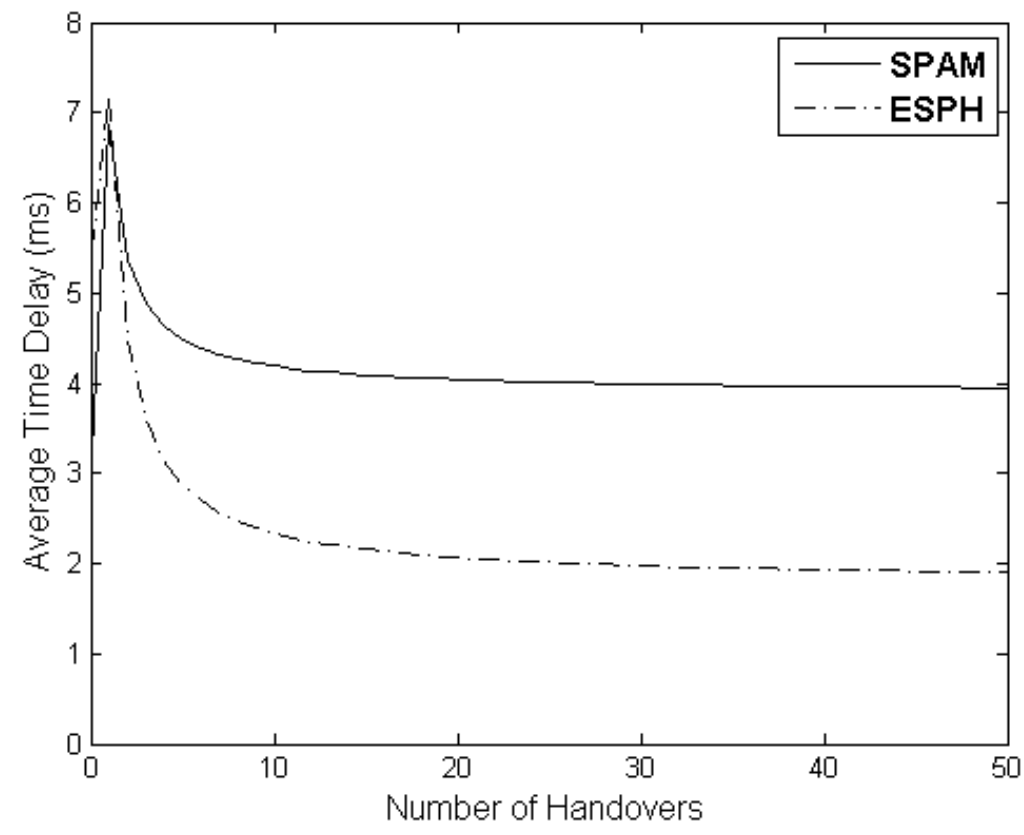

(b) Average Time Consumption of Handover Phase

Figure 4.7 Comparison of Performance Evaluation between ESPH and SPAM

\subsection{Summary}

To enhance the security functionality for the 6LoWPAN networks, the ESPH scheme has been proposed in this chapter. A hybrid cryptography has been used to provide secure information exchanges among the 6MNs, the 6MAGs, the LMA and the AAA server. The ESPH scheme enables a $6 \mathrm{MN}$ to efficiently and securely roam in the 6LoWPAN networks. The logic correctness of the proposed scheme has been proved by using PCL. The security analysis by the AVISPA has shown that the ESPH scheme is safe and can successfully prevent various malicious attacks. The performance evaluation of the ESPH scheme demonstrates that the average overall overhead incurred in a handover can be reduced when the number of handovers supported by one authentication gets larger, which can support more efficient handovers by the ESPH scheme. 


\section{Chapter 5 Secure Group Mobility Support}

\section{for 6LoWPAN Networks}

\subsection{Introduction}

In the previous chapter, a secure mobility scheme based on the PMIPv6, named ESPH, is proposed to provide protections for 6LoWPAN MNs holding limited resources. However, the ESPH as well as the PMIPv6-based solutions [45, 46, 48] reviewed in section 2.2 can only support one single node handover. To meet the needs of the future IoT, supporting a large number of devices at the same time is a prerequisite. An enhanced group mobility protocol for 6LoWPAN-based wireless body area network (WBAN) has been proposed in [82] to further reduce the number of signaling messages and lower the handover latency. Although many solutions have improved the performance of the handovers from various aspects, the security issues in 6LoWPAN networks have not been addressed much.

As for the security of group mobility, a group handover scheme [83] with privacy preservation has been proposed for the mobile WiMAX networks. And recently, some group-based handover authentication schemes [84-86] have been proposed to provide robust security protection for the Long Term Evolution (LTE) Advanced (LTE-A) networks. However, although these mechanisms aim at improving the group handover authentication, it may not be suitable to directly apply these solutions to the 6LoWPAN networks due to different system architecture and device capabilities.

In this chapter, a secure group mobility scheme (SGMS) is designed to ensure the security protections for group mobility in 6LoWPANs. To the best of our knowledge, this is the first solution targeting at the group security in 6LoWPANs. The goal of SGMS is to achieve fast inter-domain handovers, prevent unauthorized devices accessing the 6LoWPAN services and lower the handover latency. There are three phases in the SGMS scheme, which are registration phase, initialization phase, and handover phase. With the consideration of the resource constraints of the MNs, only a 
simple hash function and the AES symmetric key algorithm are adopted to enable the security supports and guarantee the authenticity of MNs when roaming in the 6LoWPAN networks. The formal verification and the simulation results have proved that the SGMS scheme has the ability to provide security functionalities and efficient handovers.

\subsection{System Background}

\subsubsection{System Architecture}

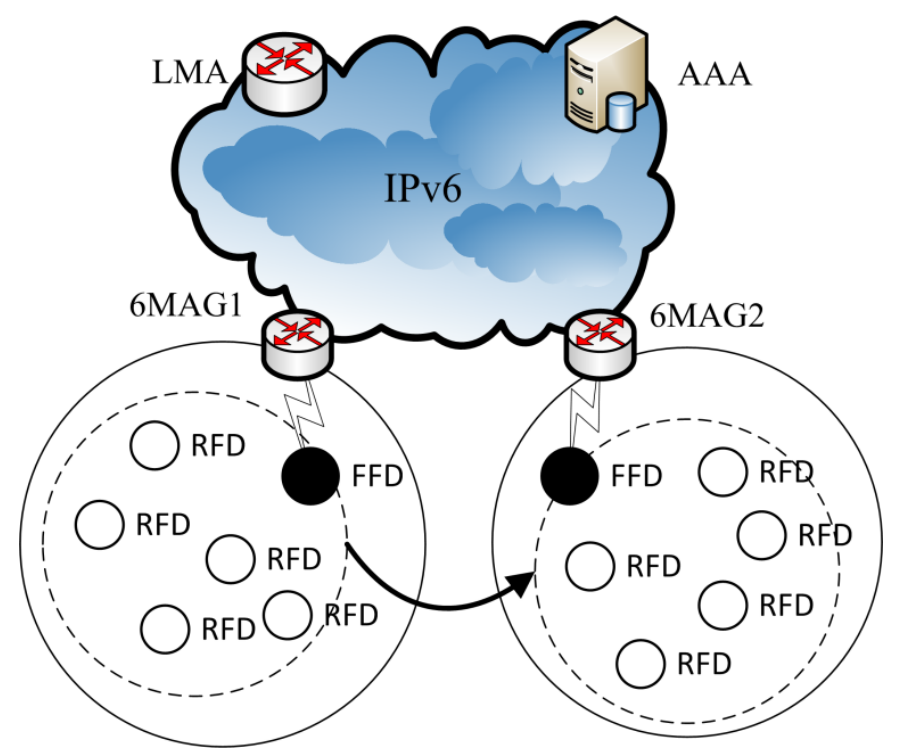

Figure 5.1 6LoWPAN System Architecture of SGMS

The 6LoWPAN system, as depicted in Figure 5.1, consists of a number of 6MNs, some 6MAGs, a LMA and a AAA server. The 6MNs, which are mobile devices used for collecting sensory information, can be FFDs or RFDs. FFDs have enough power and memory to operate as a router while RFDs can only work as hosts. The 6MNs can move across different domains within a 6LoWPAN network. The 6MAGs are responsible for tracking the movements of the $6 \mathrm{MNs}$ within their domain and sending mobility-related signaling to the LMA on behalf of the 6MNs. The LMA maintains the binding states for currently attached $6 \mathrm{MNs}$ and assigns unique network prefixes to 
registered $6 \mathrm{MNs}$, from which a $6 \mathrm{MN}$ can configure its IP addresses. Data packets destined to these addresses are routed through the LMA. The AAA server is responsible for authenticating the $6 \mathrm{MNs}$ and authorize legal devices to access the network. The policy files of the $6 \mathrm{MNs}$ are sets of configuration information stored in the AAA server. The 6MAGs, the LMA and the AAA server are connected using wired cable with high bandwidth and low bit error rates.

\subsubsection{Threat Model}

The threat model of the SGMS works based on the Dolev-Yao intruder model [65]. Under this model, the attacker may be an outsider or a legitimate principal in the system that can eavesdrop on any message transmitted through the channel. Messages can be decrypted or encrypted if the attacker obtains the corresponding secret key. Attackers are able to alter, construct, decompose, forge, replay or inject any messages and send them to any legitimate entity. Moreover, the attacker can impersonate a principal to communicate with the legal devices.

However, the adversaries are not capable of anything. It is assumed that there are certain things that an adversary is unable to do. The nonce randomly selected from a sufficiently large space cannot be guessed. The attacker also cannot retrieve the information from a given ciphertext or generate a valid ciphertext from a given plaintext without a complete and correct key. Besides, the private key of a given public key cannot be calculated.

\subsection{The SGMS Scheme}

\subsubsection{Motivation}

To provide secure mobility for group handovers in 6LoWPANs, a lightweight secure PMIPv6-based group handover scheme is designed. Most existing solutions require that an $\mathrm{MN}$ needs to perform authentication procedures each time when it joins a new domain. Therefore, the signaling overhead is quite high. To support fast interdomain handovers and provide security protections for group mobility at the same 
Table 5.1 Notations Used in Chapter 5

\begin{tabular}{cc}
\hline \hline Notation & Description \\
\hline$I D_{x}$ & Identity of $x$ \\
$T I D_{x}$ & Temporary identity of $x$ \\
$g I D$ & The identity of the group \\
$t g I D$ & The temporary identity of the group \\
$r_{x}$ & Nonce generated by $x$ \\
$T_{x}$ & Timestamp generated by $x$ \\
$K_{x \leftrightarrow y}$ & Session key established between $x$ and $y$ \\
$H$ & One-way hash function. \\
$H M A C$ & Hash-based message authentication code \\
$E_{K}(M)$ & Message $M$ is encrypted with the key $K$ \\
$D_{K}(C)$ & Ciphertext $C$ is decrypted with the key $K$ \\
\hline \hline
\end{tabular}

time, the proposed scheme enables a group of MNs to perform a full authentication with the AAA server only at the initial step. Thus, when the group of the MNs attaches to a new gateway, these MNs can be fast authenticated based on the secret values generated previously.

\subsubsection{Details of the SGMS Scheme}

The proposed SGMS scheme is designed for secure group handovers in 6LoWPAN networks based on the PMIPv6 protocol. It consists of three phases: 1) registration phase, 2) initialization phase and 3) handover phase. The notations used in this scheme are listed in Table 5.1. The IID of the 6LoWPAN devices are generated by using the method in [87] instead of using the IEEE MAC ${ }^{1}$ address. This IID generation method with the IPv6 stateless address auto-configuration mitigates the host-tracking and 
address-scanning possibilities, which is more secure and privacy preserving. It is assumed that the links among the 6MAGs, the LMA and the AAA server are connected with wired cables. Thus, the existing IPsec and IKEv2 can be implemented to secure the communication and pre-shared the secret keys. The backbone LMA and the AAA server are assumed trustworthy.

1) Registration Phase

In the deployment of a 6LoPWAN device in the network, each device needs to register with the AAA server through a secure channel. A unique identifier $I D_{i}$ and a randomly chosen integer $r 1_{i}$ are sent to the authentication server by the $6 M N_{i}$ for registration. Upon receiving the registration request, the server makes sure that this $6 \mathrm{MN}$ has not been registered and sends back a registration response with a temporary identifier (TID) $T I D_{i}$ and a nonce $r 2_{i}$. The $T I D_{i}$ will be published to the public instead of using the real identifier, which prevents the attacker from extracting device information and tracking the device. After both parties store the registration information $<I D_{i}, T I D_{i}, r 1_{i}, r 2_{i}>$ in their memory or database, a pairwise key is calculated using the function $K_{i \leftrightarrow A}=H\left(I D_{i}, I D_{A A A}, r 1_{i}\right)$. The registration procedures of SGMS are depicted in Figure 5.2.

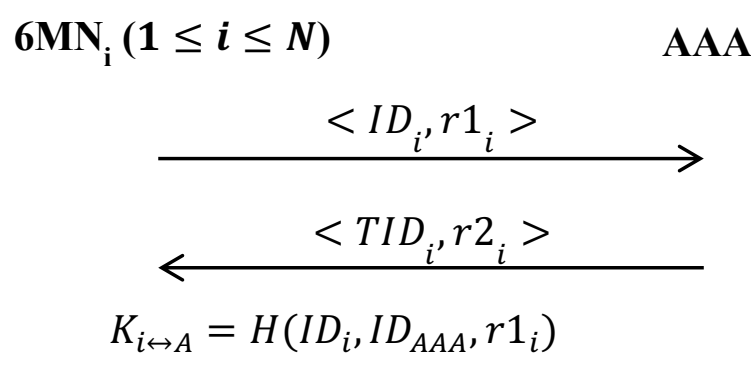

Figure 5.2 Registration Phase of SGMS

\section{2) Initialization Phase}

When a group of registered devices first join the network, initial authentication with the server is a required. In this phase, a group of the $6 \mathrm{MNs}$, the $6 \mathrm{MAG}$, the LMA and the AAA server will exchange messages to achieve a mutual initial authentication 
and key establishment as shown in Figure 5.3. The details of the initialization process are described as follows.

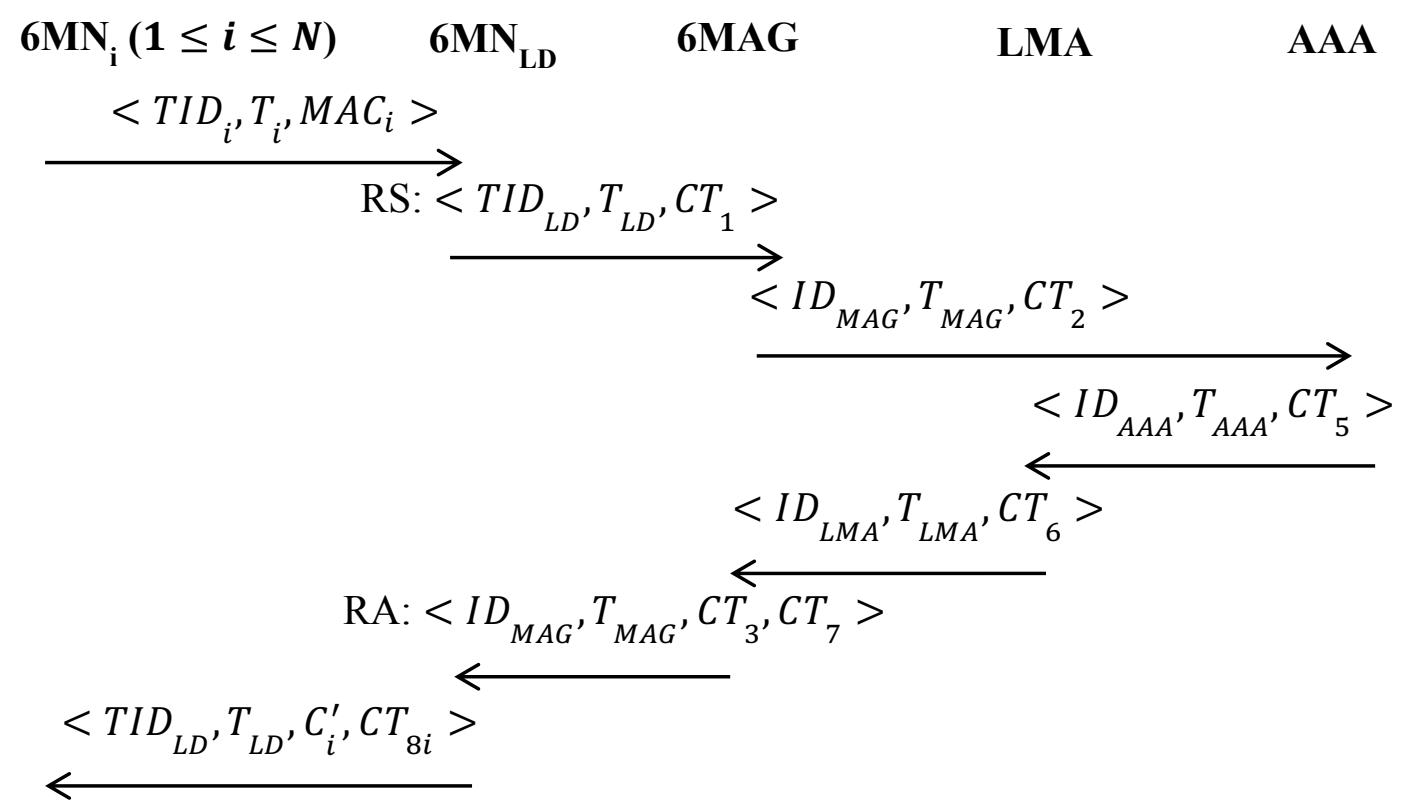

Figure 5.3 Initial Authentication Phase of SGMS

Step1: When a group of 6MNs join in a 6LoWPAN network, a group leader $6 \mathrm{MN}_{\mathrm{LD}}$ will be selected based on its capabilities. Each group member $6 \mathrm{MN}_{\mathrm{i}}(1 \leq i \leq$ $N)$ generates the $\mathrm{MAC}^{2}$ value by $M A C_{i}=H M A C_{K_{i \leftrightarrow A}}\left(T I D_{i}, T_{i}, I D_{i}, r 2_{i}\right)$. Then, all the group members send the authentication message $<T I D_{i}, T_{i}, M A C_{i}>$ to the group leader.

Step 2: After collecting all the messages, the group leader integrates its own authentication information $M A C_{L D}=H M A C_{K_{L D \leftrightarrow A}}\left(T I D_{L D}, T_{L D}, I D_{L D}, r 2_{L D}\right)$ with all the group members' messages. The integration message is computed by the equation $M_{1}=T I D_{1}, \ldots, T I D_{N}, T I D_{L D}, T_{1}, \Delta T_{2}, \ldots, \Delta T_{N}, \Delta T_{L D}$, where $\Delta T_{i}=T_{i}-T_{1}$. After that, the $6 \mathrm{MN}_{\mathrm{LD}}$ calculates the sum of the $\mathrm{MAC}^{2}$ value $M A C_{\text {sum }}=M A C_{1} \oplus \ldots \oplus M A C_{N} \oplus$ $M A C_{L D}$ and encrypts $M A C_{\text {sum }}$ and $M_{1}$ as $C T_{1}=E_{K_{L D \leftrightarrow A}}\left(M A C_{\text {sum }}, M_{1}\right)$ using the symmetric key $K_{L D \leftrightarrow A}$. The RS message $<T I D_{L D}, T_{L D}, C T_{1}>$ is sent to the 6MAG. 
Step 3: Upon receiving the message from the $6 \mathrm{MN}_{\mathrm{LD}}$, the $6 \mathrm{MAG}$ encrypts and forwards the message $<I D_{M A G}, T_{M A G}, C T_{2}=E_{K_{G \leftrightarrow A}}\left(I D_{M A G}, T_{M A G}, T I D_{L D}, C T_{1}\right)>$ to the AAA server for authentication.

Step 4: When the AAA server receives the message $<I D_{M A G}, T_{M A G}, C T_{2}>$, it first decrypts the message using the key $K_{G \leftrightarrow A}$ to verify the legitimacy of the gateway and get the ciphertext $C T_{1}$. The ciphertext $C T_{1}$ can be decrypted by using the key $K_{L D \leftrightarrow A}$ corresponding to the $T I D_{L D}$ stored in the database. After obtaining the integration message $M_{1}$, the server first derives the information of each node $\left\langle T I D_{i}, T_{i}>\right.$ from the $M_{1}$ and verify the sum of the $M^{2} C^{2}$ value $M A C_{\text {sum }}=M A C_{1} \oplus \ldots \oplus M A C_{N} \oplus M A C_{L D}$, where $M A C_{i}=H M A C_{K_{i \leftrightarrow A}}\left(T I D_{i}, T_{i}, I D_{i}, r 2_{i}\right)$. If the calculated $\mathrm{MAC}^{2}$ value and the received one are different, the server will send messages to the group leader asking for detailed $\mathrm{MAC}^{2}$ value of each member. This procedure is to investigate which member is illegal. If a $6 \mathrm{MN}$ has not been registered or the information included in the message is not corrected, a warning message will be sent to the $6 \mathrm{MAG}$ and the $6 \mathrm{MN}_{\mathrm{LD}}$ that this $6 \mathrm{MN}$ is an illegitimate device. If the calculated $\mathrm{MAC}^{2}$ value and the received one are the same, then this group of devices is proved legal. After that, the server generates two nonce $r 3$ and $r 4$, which will be further sent to the group leader for session key generation with the 6MAG or the LMA, and assigns a group ID gID and a function $f(x)=\sum_{i=1}^{N}\left(K_{i \leftrightarrow l e a d e r} \cdot \prod_{1 \leq j \leq N, j \neq i} \frac{x-T I D_{j}}{T I D_{i}-T I D_{j}}\right)$ for pairwise key established between the group member and the leader. A message $<I D_{A A A}, T_{A A A}, C T_{5}=$ $E_{K_{L \leftrightarrow A}}\left(I D_{A A A}, T_{A A A}, g I D, r 4, M_{2}, C^{\prime}, C T_{3}, C T_{4}\right)>$ is sent to the LMA, where $C T_{3}=$ $E_{K_{L D \leftrightarrow A}}\left(I D_{A A A}, T_{A A A},(r 3, r 4, g I D, f(x)) \oplus H\left(I D_{L D}, r 2_{L D}^{2}\right), C T_{4}=E_{K_{G \leftrightarrow A}}\left(I D_{A A A}, T_{A A A}\right.\right.$, $\left.I D_{M A G}, r 3\right), \quad C_{i}^{\prime}=E_{K_{i \leftrightarrow A}}\left(T I D_{L D} \oplus H\left(I D_{i}, r 2_{i}^{2}\right) \oplus K_{i \leftrightarrow L D}, g I D, H\left(g I D, K_{i \leftrightarrow L D}, T_{A A A}\right)\right.$, $C^{\prime}=C_{1}^{\prime}, \ldots, C_{N}^{\prime}$ and $M_{2}=T I D_{1}, \ldots, T I D_{N}, T I D_{L D}, I D_{1}, \ldots, I D_{N}, I D_{L D}$.

Step 5: When the LMA receives the message from the AAA server, the LMA first decrypts and verifies whether the message is truly sent from the server. If the message can be successfully decrypted, IDs of each device $T I D_{i}, I D_{i}(i=1, \ldots, N, L D)$ can be derived from $M_{2}$. The identifiers and temporary identifiers list are stored with the real group ID and nonce $r 4$ in the LMA database. The LMA assigns each device a home 
network prefix (HNP) by which the device can configure its IP address. The session key established with the $6 \mathrm{MN}_{\mathrm{LD}}$ is generated using $K_{L D \leftrightarrow L}=H\left(I D_{L D}, r 4, I D_{L M A}, g I D\right)$ based on nonce $r 4$. A temporary group ID $\operatorname{tg} I D$ is calculated by the equation $\operatorname{tg} I D=$ $H\left(r 4, I D_{L D}, T_{A A A}\right) \oplus g I D$. A message $<I D_{L M A}, T_{L M A}, C T_{6}=E_{K_{G \leftrightarrow L}}\left(I D_{L M A}, T_{L M A}\right.$, $\left.\operatorname{tg} I D, M_{3}, C T_{4}, M A C^{\prime}, C T_{3}, C^{\prime}\right)>$ is sent to the $6 \mathrm{MAG}$ by the LMA, where $M A C^{\prime}=$ $\operatorname{HMAC}\left(K_{L D \leftrightarrow L},\left(\sum_{i=1}^{N} \operatorname{TID}_{i} \oplus T I D_{L D} \oplus \sum_{i=1}^{N} H N P_{i} \oplus H N P_{L D}\right), g I D\right)$ and $M_{3}=T I D_{1}$, $\ldots, T I D_{N}, T I D_{L D}, H N P_{1}, \ldots, H N P_{N}, H N P_{L D}$.

Step 6: When receiving the message from the LMA, the 6MAG decrypts this message and verifies its validity. The nonce $r 3$ can be derived from the ciphertext $C T_{4}$ using the key $K_{G \leftrightarrow A}$. After that, the session key established between the group leader and the $6 \mathrm{MAG}$ is calculated using $K_{L D \leftrightarrow G}=H\left(T I D_{L D}, r 3, I D_{M A G}, \operatorname{tgID}\right)$. The HNP of each device can be obtained from the integration message $M_{3}$. Then, $M_{3}, M A C^{\prime}, C T_{3}$ and $C^{\prime}$ will be forwarded to the $6 \mathrm{MN}_{\mathrm{LD}}$ in the RA message $<I D_{M A G}, T_{M A G}, C T_{3}, C T_{7}=$ $E_{K_{L D \leftrightarrow G}}\left(I D_{M A G}, T_{M A G}, \operatorname{tg} I D, M_{3}, M A C^{\prime}, C^{\prime}\right)>$.

Step 7: When the $6 \mathrm{MN}_{\mathrm{LD}}$ receives the RA message, it first decrypts $C T_{3}$ using the session key $K_{L D \leftrightarrow A}$. Thus, the $6 \mathrm{MN}_{\mathrm{LD}}$ is able to obtain the nonce $r 3$ and $r 4$ from the plaintext by $\left((r 3, r 4, g I D, f(x)) \oplus H\left(I D_{L D}, r 2_{L D}^{2}\right)\right) \oplus H\left(I D_{L D}, r 2_{L D}^{2}\right)$. The function $f(x)$ used for establishing pairwise keys with each group member is stored in its memory. The key $K_{L D \leftrightarrow L}$ is generated based on the nonce $r 4$ using the equation $K_{L D \leftrightarrow L}=H\left(I D_{L D}, r 4, I D_{L M A}, g I D\right)$. After that, $M_{3}, M A C^{\prime}$ and $t g I D$ can be successfully retrieved from $C_{4}$. Thus, key $K_{L D \leftrightarrow G}$ could be recovered using $K_{L D \leftrightarrow G}=$ $H\left(T I D_{L D}, r 3, I D_{M A G}, \operatorname{tgID}\right)$ The real group ID $g I D$ and temporary group ID can be checked by $g I D=H\left(r 4, I D_{L D}, T_{A A A}\right) \oplus t g I D$. The $C_{i}^{\prime}$ and $H N P_{i}$ for each group member are extracted from the $M_{3}$. The authentication response message $<T I D_{L D}$, $T_{L D}, C_{i}^{\prime}, C T_{8 i}=E_{K_{i \leftrightarrow L D}}\left(T I D_{L D}, T_{L D}, H N P_{i}\right)>$ will be sent to each group member $6 \mathrm{MN}_{\mathrm{i}}$ after the verification of the value $M A C^{\prime}$, which ensures that the messages have not been changed during the transmission. 
Step 8: Upon receiving the authentication response from the group leader, each member decrypts $C_{i}^{\prime}$ to retrieve and verify $g I D$ and $K_{i \leftrightarrow L D}$. Thus, the $H N P_{i}$ can be recovered from the $C T_{8 i}$ using the key $K_{i \leftrightarrow L D}$.

3) Handover Phase

Most existing schemes require that the authentication procedure is performed every time when each $6 \mathrm{MN}$ attaches to a new $6 \mathrm{MAG}$, resulting in a longer handover latency and heavy workload. The proposed SGMS scheme enables a fast group handover authentication for a shorter handover delay. The handover phase is shown in Figure 5.4.

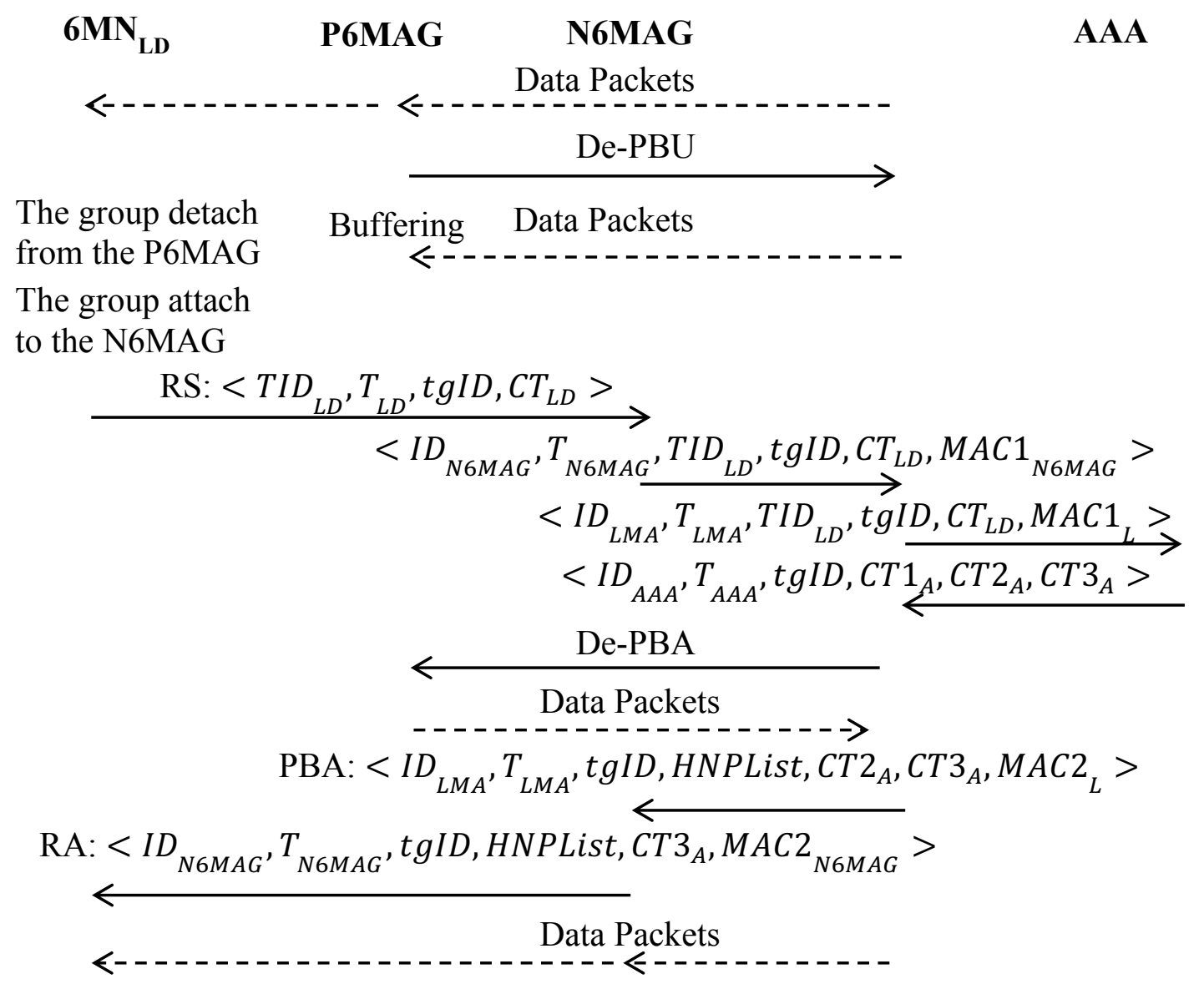

Figure 5.4 Handover Phase of SGMS

If a P6MAG detects that a group of devices has left its domain, it sends a De-PBU message to the LMA and starts buffering data packets for this group. Before entering the domain of a $\mathrm{N} 6 \mathrm{MAG}$, the $6 \mathrm{MN}_{\mathrm{LD}}$ collects the handover information from each 
member $g I D^{M A C_{i}=H M A C_{K i \leftrightarrow A}\left(T I D_{i}, I D_{i}, r 2_{i}^{j}\right)} \bmod p$, in which $j$ means $j$ th handover of this group. When this group attach to a N6MAG, a RS message $<T I D_{L D}, T_{L D}, \operatorname{tg} I D$, $C T_{L D}>$ is sent to the N6MAG, where $C T_{L D}=E_{K_{L D \leftrightarrow A}}\left(T I D_{L D}, T_{L D}, \operatorname{tg} I D, M_{H}\right)$ and the group handover information $M_{H}$ is $g I D^{M A C_{1}+\cdots+M A C_{N}+M A C_{L D}} \bmod p$. After receiving the RS message from the group leader, a PBU message $<I D_{N 6 M A G}, T_{N 6 M A G}, T I D_{L D}$, $\operatorname{tgID}, C T_{L D}, M A C 1_{N 6 M A G}=H M A C_{K_{N G G L}}\left(I D_{N 6 M A G}, T_{N 6 M A G}, T I D_{L D}, \operatorname{tg} I D, C T_{L D}\right)>$ is sent to the LMA by the N6MAG to inform that a group of 6MNs whose group ID is $\operatorname{tgID}$ is currently roaming in its domain. After checking the $\mathrm{MAC}^{2}$ value of PBU message, the LMA sends the handover information in message $<I D_{L M A}, T_{L M A}$, $T I D_{L D}, \operatorname{tgID}, C T_{L D}, M A C 1_{L}=H M A C_{K_{L \leftrightarrow A}}\left(I D_{L M A}, T_{L M A}, T I D_{L D}, \operatorname{tgID}, C T_{L D}\right)>$ to the AAA server for further verification. When the AAA server receives the verification information, it calculates the $M_{H}$ using the data stored in the database. If the received value of the $M_{H}$ and the calculated one are different, the AAA server will send a warning message to the N6MAG and the LMA to inform that this mobile group may has some illegal devices. If the received value of the $M_{H}$ and the calculated one are the same, a message $<I D_{A A A}, T_{A A A}, \operatorname{tgID}, C T 1_{A}, C T 2_{A}, C T 3_{A}>$ will be sent to the LMA, where $C T 1_{A}=E_{K_{L \leftrightarrow A}}\left(I D_{A A A}, T_{A A A}, g I D\right), C T 2_{A}=E_{K_{N G \leftrightarrow A}}\left(I D_{A A A}, T_{A A A}, \operatorname{tgID}, r 5\right)$ and $C T 3_{A}=E_{K_{L D \leftrightarrow A}}\left(I D_{A A A}, T_{A A A}, r 5 \oplus H\left(I D_{L D}, r 2_{L D}^{j \oplus 1}\right)\right)$. The LMA updates the binding cache entry for recording the N6MAG if $C T 1_{A A A}$ can be successfully decrypted. The LMA also sends a De-PBA message and a PBA message $<I D_{L M A}, T_{L M A}, t g I D$, HNPList, $C T 2_{A}, C T 3_{A}, M A C 2_{L}=H M A C_{K_{N G \leftrightarrow L}}\left(I D_{L M A}, T_{L M A}\right.$, tgID, HNPList, CT2 ${ }_{A}$, $\left.C T 3_{A}\right)>$ to the P6MAG and the N6MAG respectively. The HNPList is a list of HNPs assigned to each member for continuous communication. Upon receiving the PBA from the LMA, CT3 $3_{A}$ is forwarded in the RA message $<I D_{N 6 M A G}, T_{N 6 M A G}, \operatorname{tgID}$, HNPList, $C T 3_{A}, M A C 2_{N 6 M A G}>$, where $M A C 2_{N G M A G}=H M A C_{K_{L D \leftrightarrow N G}}\left(I D_{N 6 M A G}\right.$, $\left.T_{N 6 M A G}, \operatorname{tgID}, H N P L i s t, C T 3_{A}\right)>$ and $K_{L D \leftrightarrow N G}=H\left(T I D_{L D}, r 5, I D_{N 6 M A G}, \operatorname{tgID}\right)$. The buffered data can be transmitted back to the LMA and forwarded to the N6MAG. The N6MAG re-organizes the order of the data packets and sends them to the group leader 
in sequence. The data packets will be further assigned to the corresponding $6 \mathrm{MN}$ according to the destination address of each data. Thus, the group of MNs has successfully attached to the N6MAG to obtain the data.

\subsection{Security Analysis}

In this section, the security of the proposed SGMS scheme is analyzed from three aspects. First, the logic correctness of the SGMS by Burrows-Abadi-Needham (BAN) logic is presented. Then, a formal verification is presented using Scyther to verify the security functionalities. Further analysis of the security ability against various malicious attacks of the proposed solution is provided.

\subsubsection{Logic Proof of Handover Phase}

BAN logic [88] is the most widely used logic for analyzing the logic correctness of communication protocols. PCL and BAN logic are formal logics which support direct reasoning about the consequences of individual protocol steps without explicit reasoning about the actions of an attacker. BAN logic is the first logic to formally reason about the beliefs of an authentication protocol while PCL can prove not only the authentication properties but also other security properties of a given protocol, such as whether a shared secret is known only to the authorized entities. Since this thesis mainly focuses on authentication, PCL and BAN logic can be used for the same purpose. However, the disadvantages of PCL are that the process to prove the authentication properties is complicated and the proof will become excessively long if the number of the protocol participants increases. In contrast, the logic correctness of a protocol can be proved by only one BAN logic proof which is relatively short and simple. Therefore, BAN logic has an advantage in group authentication protocols involving a number of communication participants while PCL would be a better choice for single device authentication.

The goal of using BAN logic is to verify whether the expected result of the security protocol can be reached by deriving the beliefs of each legitimate principal participated in the authentication protocol. By BAN Logic, there are three steps of 
proving the logic correctness of a given protocol using BAN logic: 1) Idealize the protocol: By this step, the source of the messages and the plaintexts of the encrypted message will be specified. The protocol is written in the idealized form. 2) Specify the assumptions about the initial state: By this step, initial assumptions are specified, such as which two entities share the session key. 3) Derive the beliefs held by the protocol principals: Based on step 1 and 2, the conclusion on whether the security goal that both the group leader and the N6MAG believe the session key has been achieved or not will be derived using the BAN logic rules. The notations being used in BAN logic are shown in Table 5.2.

Table 5.2 Notations Used in BAN Logic

\begin{tabular}{cc}
\hline \hline Notations & Description \\
\hline$P \mid \equiv X$ & $P$ believes $X$. \\
$P \triangleleft X$ & $P$ sees $X$. \\
$P \mid \sim X$ & $P$ once said $X$. \\
$P \Rightarrow X$ & $P$ has jurisdiction over $X$. \\
$\#(X)$ & The formula $X$ is fresh. \\
$P \stackrel{K}{\leftrightarrow} Q$ & $P$ and $Q$ may communicate with each other using the \\
& shared key $K$, which is a good key. \\
$\Leftrightarrow$ & The secret formula $X$ is only known to $P$ and $Q$. \\
& The formula $X$ is encrypted with key $K$. \\
$\langle X\rangle_{K}$ & The formula $X$ is combined with secret $Y$. \\
\hline \hline
\end{tabular}

1) Idealization

By BAN logic, the first step of the logic proof is to transform the protocol into an idealization form. The plaintext and the messages, such as the first two messages of the SGMS, which do not contribute to the beliefs of the recipient, are omitted. The idealized protocol is shown as follows. 
Message 3: $L M A \rightarrow A A A: C T_{L D}, M A C 1_{L}$

Message 4: $A A A \rightarrow L M A: C T 1_{A}, C T 2_{A}, C T 3_{A}$

Message 5: LMA $\rightarrow$ N6MAG:CT2 $2_{A}, C T 3_{A}, M A C 2_{L}$

Message 6: N6MAG $\rightarrow 6 M N_{L D}: C T 3_{A}, M A C 2_{N 6 M A G}$

2) Initial state assumption

By BAN logic, in the second step of the logic proof, the initial assumptions should be stated. After the registration and initial authentication phase, the shared secret and keys are all successfully assigned to each corresponding principal. The assumptions are listed below.

A01. $6 M N_{i} \mid \equiv I D_{i}, i=1, \ldots, N, L D$

A02. $6 M N_{i} \mid \equiv g I D$

A03. $6 M N_{i} \mid \equiv \operatorname{tgID}$

A04. $6 M N_{i} \mid=6 M N_{i} \stackrel{r 2_{i}}{\Leftrightarrow} A A A$

A05. $6 M N_{i} \mid=6 M N_{i} \stackrel{K_{i \leftrightarrow A}}{\longleftrightarrow} A A A$

A06. $6 M N_{i} \mid \equiv \#\left(T_{i}\right), \#\left(T_{N 6 M A G}\right), \#\left(T_{L M A}\right), \#\left(T_{A A A}\right)$

A07. $6 M N_{j} \mid \equiv 6 M N_{j} \stackrel{K_{j \leftrightarrow L D}}{\longleftrightarrow} 6 M N_{L D}, j=1, \ldots, N$

A08. $6 M N_{L D} \mid \equiv A A A \Rightarrow 6 M N_{L D} \stackrel{r 5}{\Leftrightarrow} N 6 M A G$

A09. $N 6 M A G \mid \equiv I D_{N 6 M A G}$

A10. $N 6 M A G \mid \equiv N 6 M A G \stackrel{K_{N G \leftrightarrow L}}{\longleftrightarrow} L M A$

A11. $N 6 M A G \mid \equiv N 6 M A G \stackrel{K_{N G \leftrightarrow A}}{\longleftrightarrow A A A}$

A12. $N 6 M A G \mid \equiv \#\left(T_{i}\right), \#\left(T_{N 6 M A G}\right), \#\left(T_{L M A}\right), \#\left(T_{A A A}\right)$

A13. $N 6 M A G \mid \equiv L M A \Rightarrow t g I D$

A14. $N 6 M A G \mid \equiv A A A \Rightarrow 6 M N_{L D} \stackrel{r 5}{\Leftrightarrow} N 6 M A G$

A15. $L M A \mid \equiv I D_{i}$

A16. $L M A \mid \equiv g I D$

A17. $L M A \mid \equiv t g I D$

A18. $L M A \mid \equiv N 6 M A G \stackrel{K_{N G \leftrightarrow L}}{\longleftrightarrow} L M A$ 
A19. $L M A \mid \equiv L M A \stackrel{K_{L \leftrightarrow A}}{\longleftrightarrow} A A A$

A20. $L M A \mid \equiv \#\left(T_{i}\right), \#\left(T_{6 M A G}\right), \#\left(T_{L M A}\right), \#\left(T_{A A A}\right)$

A21. $A A A \mid \equiv I D_{i}$

A22. $A A A \mid \equiv g I D$

A23. $A A A \mid \equiv 6 M N_{i} \stackrel{r 2_{i}}{\Leftrightarrow} A A A$

A24. $A A A \mid \equiv 6 M N_{i} \stackrel{K_{i \leftrightarrow A}}{\longleftrightarrow} A A A$

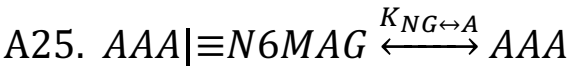

A26. $A A A$ believes $L M A \stackrel{K_{L \leftrightarrow A}}{\longleftrightarrow} A A A$

A27. $A A A \mid \equiv \#\left(T_{i}\right), \#\left(T_{6 M A G}\right), \#\left(T_{L M A}\right), \#\left(T_{A A A}\right)$

3) Belief Derivation

By BAN logic, the third step of the logic proof is to verify whether the security goal has been achieved using BAN logic rules. The beliefs held by the protocol principals are derived based on the idealized messages and initial assumptions. The rules used in this step are listed in Table 5.3.

Table 5.3 BAN Logic Rules

\begin{tabular}{|c|c|}
\hline Name & Description \\
\hline Message-Meaning Rule & $\frac{P \mid \equiv P \stackrel{K}{\leftrightarrow} Q, P \triangleleft\{X\}_{K}}{P|\equiv Q| \sim X}$ \\
\hline Nonce-Verification Rule & $\frac{P|\equiv \#(X), P| \equiv Q \mid \sim X}{P|\equiv Q| \equiv X}$ \\
\hline Jurisdiction Rule & $\frac{P|\equiv Q \Rightarrow X, P| \equiv Q \mid \equiv X}{P \mid \equiv X}$ \\
\hline Belief Conjuncatenation Rule & $\frac{P|\equiv X, P| \equiv Y}{P \mid \equiv(X, Y)}$ \\
\hline Freshness Conjuncatenation Rule & $\frac{P \mid \equiv \operatorname{fresh}(X)}{P \mid \equiv \operatorname{fresh}(X, Y)}$ \\
\hline
\end{tabular}


(1) $A A A \triangleleft\left\{T I D_{L D}, T_{L D}, \operatorname{tgID}, M_{H}\right\}_{K_{L D \oplus A}}$

(2) $A A A\left|\equiv 6 M N_{L D}\right| \sim\left(T I D_{L D}, T_{L D}, \operatorname{tg} I D, M_{H}\right)$ by (1), A05 and message meaning rule.

(3) $A A A \mid \equiv \#\left(T I D_{L D}, T_{L D}, t g I D, M_{H}\right)$ by (2), A06 and freshness conjuncatenation rule.

(4) $A A A\left|\equiv 6 M N_{L D}\right| \equiv\left(T I D_{L D}, T_{L D}, \operatorname{tgID}, M_{H}\right)$ by (2), (3) and nonce verification rule.

(5) $A A A\left|\equiv 6 M N_{i}\right| \sim g I D^{M A C_{i}}$ by (4), A05 and message meaning rule. The authentication server believes that the handover information is truly sent by each corresponding $6 \mathrm{MN}$.

(6) $L M A \triangleleft\left\{I D_{A A A}, T_{A A A}, g I D\right\}_{K_{L \leftrightarrow A}}$

(7) $L M A|\equiv A A A| \sim\left(I D_{A A A}, T_{A A A}, g I D\right)$ by (6), A19 and message meaning rule.

(8) $L M A \mid \equiv \#\left(I D_{A A A}, T_{A A A}, g I D\right)$ by (7), A20 and freshness conjuncatenation rule.

(9) $L M A|\equiv A A A| \equiv\left(I D_{A A A}, T_{A A A}, g I D\right)$ by (7), (8) and nonce verification rule.

(10) $N 6 M A G \triangleleft t g I D, T_{L M A}, M A C 2_{L}$

(11) $N 6 M A G|\equiv L M A| \sim \operatorname{tgID}$ by (10), A10 and message meaning rule.

(12) $N 6 M A G \mid \equiv \# \operatorname{tgID}$ by (11), A12 and freshness conjuncatenation rule.

(13) $N 6 M A G|\equiv L M A| \equiv \operatorname{tgID}$ by (11), (12) and nonce verification rule.

(14) $N 6 M A G \mid \equiv \operatorname{tgID}$ by (13), A13 and jurisdiction rule.

(15) $N 6 M A G \triangleleft\left\{I D_{A A A}, T_{A A A}, T I D_{L D}, \operatorname{tgID}, r 5\right\}_{K_{N G \leftrightarrow A}}$

(16) $N 6 M A G|\equiv A A A| \sim\left(I D_{A A A}, T_{A A A}, T I D_{L D}, \operatorname{tg} I D, r 5\right)$ by (15), A11 and message meaning rule.

(17) $N 6 M A G \mid \equiv \#\left(I D_{A A A}, T_{A A A}, T I D_{L D}, \operatorname{tgID}, r 5\right)$ by (16), A12 and freshness conjuncatenation rule.

(18) $N 6 M A G|\equiv A A A| \equiv\left(I D_{A A A}, T_{A A A}, T I D_{L D}, \operatorname{tg} I D, r 5\right)$ by (16), (17) and nonce verification rule.

(19) $N 6 M A G \mid \equiv r 5$ by (18), A14 and jurisdiction rule.

(20) $N 6 M A G \mid \equiv \# K_{L D \leftrightarrow N G}$ by (17) and freshness conjuncatenation rule.

(21) $N 6 M A G \mid \equiv\left\langle K_{L D \leftrightarrow N G}\right\rangle_{(r 5)}$ by (14), (19), A09 and A14. 
(22)

$6 M N_{L D} \triangleleft\left\{I D_{A A A}, T_{A A A}, r 5 \oplus H\left(I D_{L D}, r 2_{L D}^{j \oplus 1}\right)\right\}_{K_{L D \leftrightarrow A}}$

(23)

$6 M N_{L D}|\equiv A A A| \sim\left(I D_{A A A}, T_{A A A}, r 5 \oplus H\left(I D_{L D}, r 2_{L D}^{j \oplus 1}\right)\right)$ by (22), A05 and message meaning rule.

(24) $6 M N_{L D} \mid \equiv \#\left(I D_{A A A}, T_{A A A}, r 5 \oplus H\left(I D_{L D}, r 2_{L D}^{j \oplus 1}\right)\right)$ by (23), A06 and freshness conjuncatenation rule.

(25) $6 M N_{L D}|\equiv A A A| \equiv\left(I D_{A A A}, T_{A A A}, r 5 \oplus H\left(I D_{L D}, r 2_{L D}^{j \oplus 1}\right)\right)$ by (23), (24) and nonce verification rule.

(26) $6 M N_{L D} \mid \equiv r 5$ by (25), A08 and jurisdiction rule.

(27) $6 M N_{L D} \mid \equiv \# K_{L D \leftrightarrow N G}$ by (24) and freshness conjuncatenation rule.

(28) $6 M N_{L D} \triangleleft M A C 2_{N 6 M A G}$

(29) $6 M N_{L D}|\equiv N 6 M A G| \equiv K_{L D \leftrightarrow N G}$

(30) $6 M N_{L D} \mid \equiv K_{L D \leftrightarrow N G}$ by (29)

By verifying the $\mathrm{MAC}^{2}$ value sent from the N6MAG using the newly generated key $K_{L D \leftrightarrow N G}$, this ensures that the group leader believes that the N6MAG believes the key, and also the group leader believes the key. Thus, from (21) and (30), it can be derived that both the group leader and the N6MAG believe the key $K_{L D \leftrightarrow N G}$.

\subsubsection{Formal Verification}

Scyther [89] is an automatic verification tool designed for the formal analysis of security protocols. Although AVISPA is widely used to verify the security protocols and has a good feature that it supports exponential and XOR operations, the verification time will exponentially increase with the growing number of protocol runs or algebraic operations while Scyther has lower verification time in this case. Therefore, Scyther has an advantage in verifying group authentication protocols while AVISPA is more applicable for single device authentication. It is assumed that all cryptographic functions are perfect and the plaintext cannot be extracted unless someone has the corresponding secret key. The input language of Scyther is Security Protocol Language Description (SPDL) used for describing the protocols constructed by the user, which is defined by a set of roles, events and various terms. The intended security properties are 
verified by using claim events. For example, $\operatorname{claim}(A$, Secret, $r)$ means that $r$ is claimed to be secret in role $A$ specification. The adversary model employed in Scyther is based on the Dolev-Yao adversary model.

There are five basic roles in the handover phase of the SGMS, which are the group member $6 \mathrm{MN}_{\mathrm{i}}$, the group leader $6 \mathrm{MN}_{\mathrm{LD}}$, the $\mathrm{N} 6 \mathrm{MAG}$, the LMA and the AAA server. The security goals of SGMS are defined, by which the keys are kept secret among the corresponding roles. It assumed that there are two group members in the protocol.

\begin{tabular}{|c|c|c|c|c|c|}
\hline \multicolumn{5}{|c|}{ Scyther results : verify } & \multirow{3}{*}{$\begin{array}{l}\text { Comments } \\
\text { No attacks within bounds }\end{array}$} \\
\hline \multicolumn{4}{|c|}{ Claim } & Status & \\
\hline \multirow[t]{17}{*}{ SGMS } & MN1 & SGMS,MN11 & Secret R11 & Ok & \\
\hline & & SGMS,MN12 & Secret R21 & Ok & No attacks within bounds. \\
\hline & MN2 & SGMS,MN21 & Secret R12 & Ok & No attacks within bounds. \\
\hline & & SGMS,MN22 & Secret R22 & Ok & No attacks within bounds. \\
\hline & LD & SGMS,LD1 & Secret gID & Ok & No attacks within bounds. \\
\hline & & SGMS,LD2 & Secret R1j & Ok & No attacks within bounds. \\
\hline & & SGMS,LD3 & Secret $R 2 \mathrm{j}$ & Ok & No attacks within bounds. \\
\hline & & SGMS,LD4 & Secret R5 & Ok & No attacks within bounds. \\
\hline & & SGMS,LD5 & SKR h(TIDj,R5,IDg,tgID) & Ok & No attacks within bounds. \\
\hline & NMAG & SGMS,NMAG1 & Secret R5 & Ok & No attacks within bounds. \\
\hline & & SGMS,NMAG2 & SKR h(TIDj,R5,IDg,tgID) & Ok & No attacks within bounds. \\
\hline & LMA & SGMS,LMA1 & Secret gID & Ok & No attacks within bounds. \\
\hline & AAA & SGMS,AAA1 & Secret R5 & Ok & No attacks within bounds. \\
\hline & & SGMS,AAA2 & Secret R11 & $\mathbf{O k}$ & No attacks within bounds. \\
\hline & & SGMS,AAA3 & Secret R12 & Ok & No attacks within bounds. \\
\hline & & SGMS,AAA4 & Secret R1j & ok & No attacks within bounds. \\
\hline & & SGMS,AAA5 & Secret gID & $\mathbf{~} \mathbf{k}$ & No attacks within bounds. \\
\hline
\end{tabular}

Figure 5.5 Output Results of Fast Authentication in Handover Phase 
More group members can be easily extended in Scyther but may result in longer analysis time. Although Scyther does not support some mathematical operations, such as XOR and exponential function, there exists a helper protocol to approximately realize those functions, which has no effect on the output result of the analysis. The proposed handover phase is coded in SPDL and analyzed by Scyther. As shown in Figure 5.5, the result of SGMS demonstrates that the confidential keys, numbers or IDs are still kept secret and there is no attack found within bounded sessions under the claims as specified.

\subsubsection{Ability Against Malicious Attacks}

In this section, detailed explanations on the ability against various malicious attacks of the proposed solution are provided. The comparison of properties of authentication protocols are given in Table 5.4.

1) Replay Attacks

A replay attack is an action to maliciously resend the previously obtained messages to a receiver for extracting confidential information from the response message. By the SGMS, since every message is sent with a timestamp, the receiver can compare the received timestamp with the current time. If the timestamp is expired, namely the time difference is greater than a defined time threshold, that message should be ignored. If the timestamp is altered or deleted, the message will still be ignored unless the adversary is able to change the timestamp in the ciphertext with the corresponding secret key.

\section{2) MITM Attacks}

An MITM attack is an action to alter or delete the messages during transmission so that the two original communication parties still believe that they directly communicate with each other while they are communicating with the adversary. By the SGMS, since the $\mathrm{MAC}^{2}$ and hash function are used to protect the integration of the messages, this kind of attack can be prevented. Although the attacker can change the ciphertext during transmission, the ciphertext cannot be successfully decrypted by the receiver with the right key. Thus, the invalid messages should be ignored. 
3) Impersonation Attacks

An impersonation attack is an action to disguise as a legitimate communication party by an adversary. By the SGMS, all legal devices have registered with the AAA server before the deployment in the system. An attacker can successfully impersonate a legal device unless it has the confidential information or secret keys of a registered device, otherwise the server will compare the secret information with the stored one in the database and will send warning messages to the LMA and the MAG if the information is different.

Table 5.4 Comparison of Properties of Authentication Protocols

\begin{tabular}{|c|c|c|c|c|c|}
\hline & SAKES & SPAM & EAKES6Lo & ESPH & SGMS \\
\hline Group Authentication & $x$ & $x$ & $x$ & $x$ & $\checkmark$ \\
\hline Mobility & $x$ & $\checkmark$ & $\checkmark$ & $\checkmark$ & $\checkmark$ \\
\hline PMIPv6 & $x$ & $\checkmark$ & $x$ & $\checkmark$ & $\checkmark$ \\
\hline Resource Constrained Devices & $\checkmark$ & $\checkmark$ & $\mathrm{P}$ & $\checkmark$ & $\checkmark$ \\
\hline Against Replay Attack & $x$ & $x$ & $\checkmark$ & $\checkmark$ & $\checkmark$ \\
\hline Against MITM Attack & $\checkmark$ & $x$ & $\checkmark$ & $\checkmark$ & $\checkmark$ \\
\hline Against Impersonation Attack & $\checkmark$ & $x$ & $\checkmark$ & $\checkmark$ & $\checkmark$ \\
\hline Against Privileged Insider Attack & $x$ & $x$ & $\mathrm{P}$ & $\mathrm{P}$ & $\mathrm{P}$ \\
\hline Against Sybil Attack & $\checkmark$ & $x$ & $\checkmark$ & $\checkmark$ & $\checkmark$ \\
\hline Against Compromised Attack & $x$ & $x$ & $\mathrm{P}$ & $\mathrm{P}$ & $\mathrm{P}$ \\
\hline Formal Verification & $x$ & $x$ & $\checkmark$ & $\checkmark$ & $\checkmark$ \\
\hline
\end{tabular}

x :fully supported; $\checkmark$ : not supported; P: partially supported. 


\section{4) Privileged Insider Attacks}

An insider attack is an action to steal confidential information or inject false data by a legal insider of the network. By the SGMS, the messages are all protected by the pairwise key of two communication parties. Therefore, the secret values of other parties are difficult to extract if the attacker does not have the right secret key. Moreover, the confidential information, such as the real identity, is only known by the MN itself and the LMA or the AAA. Since the LMA and the AAA server are assumed to be trustworthy, these two parties are not able to leak the confidential values of each device. Thus, the insider can only obtain the messages sent to it and is unable to derive other devices' information.

5) Sybil Attacks

A Sybil attack is an action to forge multiple identities used for disguising a large number of legal but non-existed devices. The goal of this kind of attack is to cheat the $6 \mathrm{MAG}$ and the LMA that these non-existed devices are communicating in the domain and to consume much network resources which may result in DoS attacks. Since the registration with the AAA server is a must to each device, devices with fake identities and secret values cannot pass the authentication with the server. Therefore, Sybil attacks can be prevented in SGMS.

\subsection{Performance Evaluation}

\subsubsection{Computational Overhead}

In this section, the computational overhead of each cryptographic operation is analyzed and conducted on Intel(R) Core(TM) i5-2400 CPU @ 3.10 GHz, 4 GB RAM, Windows 7 (32-bit). The configuration of parameters listed in Table 5.5 are unified for the comparison purposes. The applied cryptographic algorithms employed in the simulation are hash function SHA- 256, MAC ${ }^{2}$ function HMAC-SHA- 256 and symmetric encryption AES-CBC-256. 
Table 5.5 Setting of Parameters of SGMS

\begin{tabular}{cc|cc}
\hline \hline Parameters & Size (bytes) & Parameters & Size (bytes) \\
\hline ID & 8 & Timestamp & 8 \\
TID & 8 & HNP & 8 \\
Nonce & 8 & Key length & 32 \\
\hline \hline
\end{tabular}

Although the SGMS scheme is proved safe and is able to prevent the attacks under the previously defined threat model, other unknown types of attacks or some unforeseen situations may result in the interruption of the execution of the SGMS. Thus, to simulate the performance of the SGMS scheme, it is assumed that an interruption, namely an attack, may randomly occur at any step of the execution of the SGMS scheme to stop its running. If the number of successful attacks is increasing, the average time consumption of a complete execution of the SGMS scheme will take longer. The comparison of the time consumption for the authentication in handovers between the SGMS scheme and the SPAM scheme is shown in Figure 5.6 (a). The number of attacks used in the simulation is set to 10000 .

It is shown that although the SPAM scheme seems that it causes less computational overhead with fewer group devices in the initial authentication listed in Table 5.6, the time consumption of the SPAM is much higher than that of the SGMS when dozens of 6LoWPAN devices are roaming in the network. Therefore, the SGMS scheme can achieve much better performance.

Table 5.6 Comparison of Computational Cost of Initial Authentication

\begin{tabular}{cc}
\hline \hline & Computational Cost $(\mathrm{ms})$ \\
\hline SGMS for a group of 10 nodes & 48.139 \\
SGMS for a group of 50 nodes & 63.111 \\
SPAM for a group of 10 nodes & 21.440 \\
SPAM for a group of 50 nodes & 107.205 \\
\hline \hline
\end{tabular}




\subsubsection{Transmission Overhead}

Since the transmission overhead is much higher than the local computational overhead for resource constrained 6LoWPAN devices, the size of the packets transmitted over the network should be as small as possible to save the energy and prolong the service life of the entire network. The energy cost of transmission of the TelosB sensor refers to the simulation results in [90] that the energy consumption for transmitting and receiving are $0.72 \mu \mathrm{J} / \mathrm{bit}$ and $0.81 \mu \mathrm{J} / \mathrm{bit}$ respectively. Since the $6 \mathrm{MAG}$, the LMA and the AAA are powerful devices connected using wired cables, the energy cost of transmitting a message is trivial compared to those battery-operated 6LoWPAN devices. Here, only the data packets transmitted using the wireless communication are considered, which means that only the messages transmitted among the group members, the group leader and the $6 \mathrm{MAG}$ are considered. Based on the frame format defined in $[9,32,33,91]$ and the RA/RS header defined in $[66,92,93]$, it is assumed that the format of the packet used for the simulation contains 8-byte PHY preamble, 21-byte MAC ${ }^{1}$ header, 6-byte compressed IPv6 and UDP header, 3-byte fragmentation header, 26-byte RA header and 22-byte RS header. A comparison of total energy cost of fast authentication in a handover is presented in Table 5.7. The results listed in the table demonstrates that the energy cost of the SGMS is considerably less that the SPAM with the same number of group members. If the malicious attacks are taken into account, the energy cost of the SGMS and the SPAM are shown in Figure $5.6(\mathrm{~b})$.

Table 5.7 Comparison of Energy Cost of Fast Authentication in Handover Phase

\begin{tabular}{cc}
\hline \hline & Energy Cost (mJ) \\
\hline SGMS for a group of 10 nodes & 9.621 \\
SGMS for a group of 50 nodes & 39.682 \\
SPAM for a group of 10 nodes & 37.454 \\
SPAM for a group of 50 nodes & 187.272 \\
\hline \hline
\end{tabular}




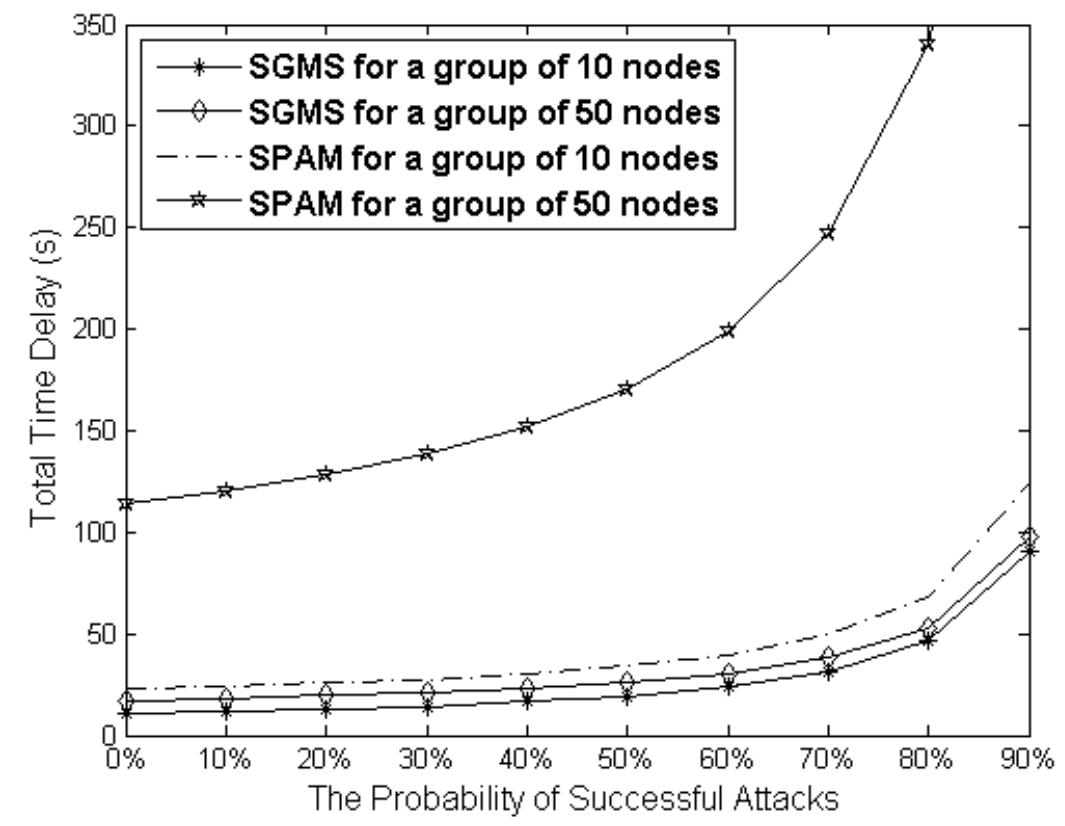

(a) Total Time Consumption of the Fast Authentication in Handovers

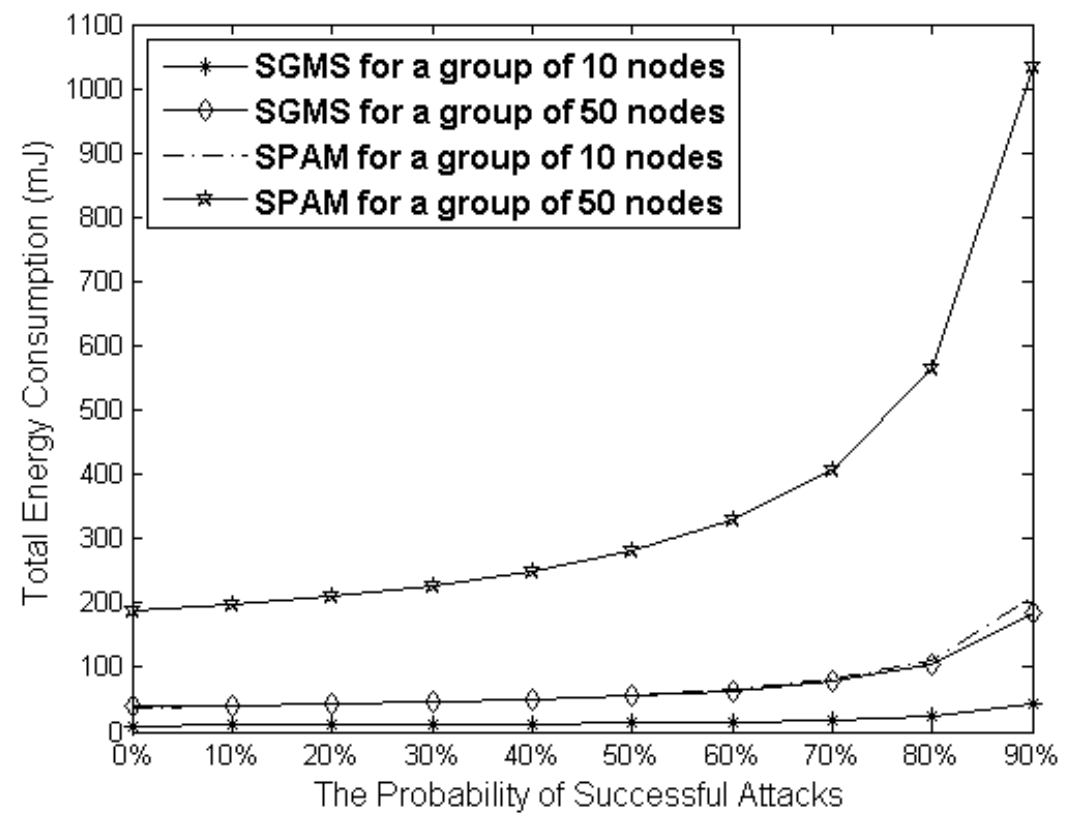

(b) The Energy Consumption of the Fast Authentication in Handovers

Figure 5.6 Comparison of Performance Evaluation between SGMS and SPAM 


\subsection{Summary}

To enhance the security functionality and efficiency of the 6LoWPAN networks, the SGMS scheme has been proposed in this chapter to support secure and seamless handovers for a group of resource constrained 6LoWPAN devices. To the best of our knowledge, this is the first secured group handover solution in the 6LoWPAN networks. The formal verification by the Scyther has verified that the SGMS scheme is safe under

the defined threat model and the security analysis has shown that it holds the abilities to prevent various malicious attacks. The performance evaluation of the proposed scheme has demonstrated that the average overall computational overhead is far less, which can support more efficient handovers by the SGMS scheme. 


\section{Chapter 6 Summary and Future Works}

In this chapter, the contributions of this thesis are summarized and the future research works are proposed.

\subsection{Contributions}

In this thesis, several important issues for 6LoWPAN network are investigated, including the architecture of the 6LoWPAN, the security issues and vulnerabilities, and mobility support for 6LoWPAN devices. The objective is to explore the inherent properties of 6LoWPAN devices and provide appropriate security protections for both static and mobile devices, so that the security of the resource constrained $\mathrm{MN}$ can be enabled while moving within the network. The major contributions of this thesis are summarized as follows:

- First, an enhanced authentication and key establishment scheme is designed for the M2M communications in 6LoWPAN networks (EAKES6Lo). Considering the resource constraints at the 6LoWPAN devices, a hybrid cryptography approach is employed in this scheme to provide the security protection for both the static and the mobile devices. When a device, whether it is static or mobile, newly joins the network, a full authentication is performed to validate the legitimacy of the device and to establish session keys. For MNs, a handover ticket is generated to achieve a fast authentication when performing handovers so that the full authentication process will only be performed once the ticket is expired, which significantly improves the handover efficiency. The security analysis has shown that the proposed scheme safe under the defined attack model. The performance evaluation demonstrates that the fast authentication process during handover achieves less computational and transmission overhead.

- Second, to further improve the handover efficiency and reduce the packet loss while moving among different domains, a secure PMIPv6-based handover 
scheme (ESPH) is designed to enable a resource constrained 6LoWPAN device to efficiently and securely roam in the 6LoWPAN networks. With the consideration of the limited power of a device, only simple symmetric encryption and hash function are adopted to guarantee the security supports for 6MNs when moving in the 6LoWPAN networks. By this proposal, a secret key chain is generated based on the directions of movement of a $6 \mathrm{MN}$ to establish secure and seamless connections with different 6MAGs. Thus, the full authentication of $6 \mathrm{MNs}$ is avoided each time when they are roaming among 6MAGs while keeping security. The security proof and the formal verification prove that the proposed scheme could prevent many malicious attacks. Besides, the average overall overhead incurred in a handover can be reduced when the number of handovers supported by one authentication gets larger, which can support more efficient handovers by this scheme.

- Third, to meet the future demand of supporting massive devices in the network, a fast and secure group handover scheme (SGMS) based on PMIPv6 is proposed to support seamless handovers for a group of resource constrained 6LoWPAN devices. The goal of the proposed scheme is to achieve fast and seamless handovers while preventing confidential data from being accessed by the attackers. The security analysis has shown that the SGMS has the abilities to prevent aganist various malicious attacks under the defined threat model. The performance evaluation has demonstrated that the average overall computational overhead of the proposed group mobility scheme is far less than the single node authentication, which can support more efficient handovers.

\subsection{Future Research Works}

Although the proposed schemes presented in the previous chapters have already made big progress in secure 6LoWPAN networks, there still are some research directions on security issues of the 6LoWPAN network which are need to be explored in the future. 
- With the increasing requirements for the mobile services, mobility support for 6LoWPAN has the advantage of realizing new and more complex applications and functionalities that enable sensor nodes to transmit the data while moving. For example, to continuously monitor the patients' health conditions, various sensor devices can be worn on the patients while they are moving in the hospital. To provide seamless handovers for 6LoWPAN MNs with limited resources, security mechanisms can adopt mobility support schemes such as network-based IP mobility management which minimizes the signaling overhead and energy consumption of the handover process without requiring the participation of the MN. However, security issues can be caused during the move and must be considered. The 6LoWPAN devices are exposed to attacks that compromise sensitive data and privacy. To tackle these problems, it is necessary to provide optimized lightweight security services like authentication, authorization, integrity and confidentiality to protect the resource constrained 6LoWPAN devices.

- With the rapid development of IoT, the number of sensing devices in the 6LoWPAN network increases quickly. When a large number of 6LoWPAN mobile devices simultaneously access the network with traditional method, each device needs to implement an independent authentication procedure. The massive authentication requests would bring large amount of signaling to the network, which could occupy lots of network resources and lead to network congestion as well. In order to guarantee the QoS and achieve simultaneous authentication of 6LoWPAN devices efficiently, more efficient group authentication methods are required to support group mobility. The group authentication, which groups multiple users that in the same domain or have the same behavior together and authenticates them as a whole at one time, could save vast network signaling resource for the future 6LoWPAN networks. Based on fast group authentication, group mobility with less handover delay and pack loss can be achieved. However, secure group mobility is still immature and is not specified in the standard. The practical and reliable group 
mobility mechanisms are still needed to solve problems such as identifying the illegitimate device and trust between group member and leader. What's more, since many 6LoWPAN devices have limited resources, secure group mobility scheme should be lightweight and efficient while retaining security.

- Key management including the generation, exchange, storage, use, and replacement of keys is critical to the security of a 6LoWPAN network in the network domain. If keys are tampered or forged, private information may be leaked to attackers which could lead to severe security issue. Since 6LoWPAN devices have limited resources and lack of physical protection in an unattended environment, a safer and stronger key management mechanism must be adopted to provide confidentiality and integrity for exchanged messages in 6LoWPAN network. However, some of the most commonly used key exchange technologies may not be applicable in 6LoWPANs due to the features of these resource constrained devices. For example, the trusted-server scheme relies on an online trusted server which arranges keys in the network. It is resilient to node compromise since each 6LoWPAN node has a unique secret key communicating with the server. But, there is usually no guarantee that the communication between 6LoWPAN nodes and the trusted server is seamless. If the trusted server is compromised by an attacker, the information of the whole network is exposed. Besides, key management schemes based on asymmetric cryptography may also be not suitable for most of the 6LoWPAN networks due to large energy consumption and memory requirements. A more secure, reliable and efficient key management solution for heterogeneous 6LoWPAN devices is required.

In summary, with the development of 6LoWPAN technology, the existing security mechanisms are inadequate and need to be improved to make the system more secure. In future, new security threats may appear, and solutions, such as key management, secure routing and data encryption, may also be developed to cope with security and privacy issues. 


\section{List of Publications}

[1] Yue Qiu and Maode Ma, "A Mutual Authentication and Key Establishment Scheme for M2M Communication in 6LoWPAN Networks," IEEE Transactions on Industrial Informatics, vol. 12, no. 6, pp. 2074-2085, Dec. 2016.

[2] Yue Qiu, Maode Ma and Xilei Wang, "A Proxy Signature-based Handover Authentication Scheme for LTE Wireless Networks," Journal of Network and Computer Applications, vol. 83, pp. 63-71, Apr. 2017.

[3] Yue Qiu and Maode Ma, "An Authentication and Key Establishment Scheme to Enhance Security for M2M in 6LoWPANs," Proceedings of 2015 IEEE International Conference on Communication Workshop (ICCW), London, pp. 2671-2676, 2015.

[4] Yue Qiu and Maode Ma, "A PMIPv6-based Secured Mobility Scheme for 6LoWPAN," Proceedings of 2016 IEEE Global Communications Conference (GLOBECOM), Washington, DC, pp. 1-6, 2016.

[5] Yue Qiu and Maode Ma, "A Secure PMIPv6-based Group Mobility Scheme for 6LoWPAN Networks," Proceedings of 2017 IEEE International Conference on Communication (ICC), Paris, France, pp. 1-6, 2017.

[6] Yue Qiu and Maode Ma, "Security Issues and Approaches in M2M Communications," Securing Cyber-Physical Systems, A. K. Pathan, Ed., Florida: CRC Press, pp. 259-278, 2015.

[7] Yue Qiu and Maode Ma, "An Efficient and Secure PMIPv6-based Handover Scheme for 6LoWPAN Networks," Journal of Network and Computer Applications (in review).

[8] Yue Qiu and Maode Ma, "Secure Group Mobility Support for 6LoWPAN Networks," IEEE Transactions on Industrial Informatics (in review).

[9] Yue Qiu, Maode Ma and Shuo Chen, “An Anonymous Authentication Scheme for Multi-Domain Machine to Machine Communication in Cyber-Physical Systems," Computer Networks (in review) 


\section{Bibliography}

[1] Y. Zhang, W. Duan and F. Wang, "Architecture and Real-Time Characteristics Analysis of the Cyber-Physical System," Proceedings of 2011 IEEE 3rd International Conference on Communication Software and Networks (ICCSN), pp. 317-320, 2011.

[2] L. Sha, S. Gopalakrishnan, X. Liu and Q. Wang, "Cyber-Physical Systems: A New Frontier," Proceedings of 2008 IEEE International Conference on Sensor Networks, Ubiquitous, and Trustworthy Computing (SUTC), pp. 1-9, 2008.

[3] A. A. Cardenas, S. Amin and S. Sastry, "Secure Control: Towards Survivable Cyber-Physical Systems," Proceedings of 2008 The 28th International Conference on Distributed Computing Systems (ICDCS) Workshops, pp. 495$500,2008$.

[4] I. Stojmenovic, "Machine-to-Machine Communications With In-Network Data Aggregation, Processing, and Actuation for Large-Scale Cyber-Physical Systems," IEEE Internet of Things Journal, vol. 1, no. 2, pp. 122-128, 2014.

[5] J. Song, A. Kunz, M. Schmidt and P. Szczytowski, "Connecting and Managing M2M Devices in the Future Internet," Mobile Networks and Applications, vol. 19, no. 1, pp. 4-17, 2014.

[6] Machine-to-Machine communications (M2M); Functional architecture ETSI TS $102690 \mathrm{~V} 2.1 .1,2013$.

[7] O. Elloumi and C. Forlivesi, "ETSI M2M Services Architecture," M2M Communications, D. Boswarthick, O. Elloumi, and O. Hersent, Eds., Hoboken, NJ: Wiley, pp. 95-140, 2012.

[8] J. W. Hui and D. E. Culler, "IPv6 in Low-Power Wireless Networks," Proceedings of the IEEE, vol. 98, no. 11, pp. 1865-1878, 2010.

[9] IEEE Standard for Low-Rate Wireless Networks, IEEE Std 802.15.4-2015, 2016.

[10] X. Ma and W. Luo, "The Analysis of 6LowPAN Technology," Proceedings of 2008 IEEE Pacific-Asia Workshop on Computational Intelligence and Industrial Application, pp. 963-966, 2008.

[11] Z. Sheng, S. Yang, Y. Yu, A. V. Vasilakos, J. A. Mccann and K. K. Leung, "A Survey on the IETF Protocol Suite for the Internet of Things: Standards, Challenges, and Opportunities," IEEE Wireless Communications, vol. 20, no. 6, pp. 91-98, 2013.

[12] N. Kushalnagar, G. Montenegro and C. Schumacher, "IPv6 over Low-Power Wireless Personal Area Networks (6LoWPANs): Overview, Assumptions, Problem Statement, and Goals," IETF RFC 4919, 2007. 
[13] E. Kim, D. Kaspar and J. Vasseur, "Design and Application Spaces for IPv6 over Low-Power Wireless Personal Area Networks (6LoWPANs)," IETF RFC 6568, 2012.

[14] L. Catarinucci, D. d. Donno, L. Mainetti, L. Palano, L. Patrono, M. L. Stefanizzi and L. Tarricone, "An IoT-Aware Architecture for Smart Healthcare Systems," IEEE Internet of Things Journal, vol. 2, no. 6, pp. 515-526, 2015.

[15] F. Touati, A. B. Mnaouer, O. Erdene-Ochir, W. Mehmood, A. Hassan and B. Gaabab, "Feasibility and Performance Evaluation of a 6LoWPAN-enabled Platform for Ubiquitous Healthcare Monitoring," Wireless Communications and Mobile Computing, vol. 16, no. 10, pp. 1271-1281, 2016.

[16] P. B. Val, M. G. Valls and M. B. Cuñado, "A Simple Data-Muling Protocol," IEEE Transactions on Industrial Informatics, vol. 10, no. 2, pp. 895-902, 2014.

[17] S. Misra, S. Goswami, C. Taneja, A. Mukherjee and M. S. Obaidat, "A PKI Adapted Model for Secure Information Dissemination in Industrial Control and Automation 6LoWPANs," IEEE Access, vol. 3, pp. 875-889, 2015.

[18] A. Kamilaris, V. Trifa and A. Pitsillides, "HomeWeb: An Application Framework for Web-based Smart Homes," Proceedings of 2011 18th International Conference on Telecommunications, pp. 134-139, 2011.

[19] D. Ş. Tudose, A. Voinescu, M. T. Petrăreanu, A. Bucur, D. Loghin, A. Bostan and N. Ţăpuş, "Home Automation Design using 6LoWPAN Wireless Sensor Networks," Proceedings of 2011 International Conference on Distributed Computing in Sensor Systems and Workshops (DCOSS), pp. 1-6, 2011.

[20] A. Fachechi, L. Mainetti, L. Palano, L. Patrono, M. L. Stefanizzi, R. Vergallo, P. Chu and R. Gadh, "A New Vehicle-to-Grid System for Battery Charging Exploiting IoT Protocols," Proceedings of 2015 IEEE International Conference on Industrial Technology (ICIT), pp. 2154-2159, 2015.

[21] V. Nagaraj, V. Kiran and K. V. Padmaja, "6LoWPAN based Intelligent Transport System for Surveillance - A Model of Ubiquitous Computing Technology," Proceedings of 2016 Eighth International Conference on Ubiquitous and Future Networks (ICUFN), pp. 801-803, 2016.

[22] M. Bouaziz and A. Rachedi, "A Survey on Mobility Management Protocols in Wireless Sensor Networks based on 6LoWPAN Technology," Computer Communications, vol. 74, no. C, pp. 3-15, 2016.

[23] D. Johnson, C. Perkins and J. Arkko, "Mobility Support in IPv6," IETF RFC 3775, 2004.

[24] H. Soliman, C. Castelluccia, K. ElMalki and L. Bellier, "Hierarchical Mobile IPv6 (HMIPv6) Mobility Management," IETF RFC 5380, 2008.

[25] R. Koodli, "Mobile IPv6 Fast Handovers," IETF RFC 5568, 2009.

[26] S. Gundavelli, K. Leung, V. Devarapalli, K. Chowdhury and B. Patil, "Proxy 
Mobile IPv6," IETF RFC 5213, 2008.

[27] V. Devarapalli, R. Wakikawa, A. Petrescu and P. Thubert, "Network Mobility (NEMO) Basic Support Protocol," IETF RFC 3963, 2005.

[28] S. Park, K. Kim, W. Haddad, S. Chakrabarti and J. Laganier, "IPv6 over Low Power WPAN Security Analysis," IETF draft-daniel-6lowpan-security-analysis05, 2011.

[29] A. Le, J. Loo, A. Lasebae, M. Aiash and Y. Luo, "6LoWPAN: A Study on QoS Security Threats and Countermeasures using Intrusion Detection System Approach," International Journal of Communication Systems, vol. 25, no. 9, pp. 1189-1212, 2012.

[30] C. Hennebert and J. D. Santos, "Security Protocols and Privacy Issues into 6LoWPAN Stack: A Synthesis," IEEE Internet of Things Journal, vol. 1, no. 5, pp. 384-398, 2014.

[31] J. Granjal, E. Monteiro and J. S. Silva, "Security for the Internet of Things: A Survey of Existing Protocols and Open Research Issues," IEEE Communications Surveys \& Tutorials, vol. 17, no. 3, pp. 1294-1312, 2015.

[32] J. Hui and P. Thubert, "Compression Format for IPv6 Datagrams over IEEE 802.15.4-Based Networks," IETF RFC 6282, 2011.

[33] G. Montenegro, N. Kushalnagar, J. Hui and D. Culler, "Transmission of IPv6 Packets over IEEE 802.15.4 Networks," IETF RFC 4944, 2007.

[34] D. Thaler, "Privacy Considerations for IPv6 Adaptation Layer Mechanisms," IETF draft-ietf-6lo-privacy-considerations-04, 2016.

[35] R. Lu, X. Li, X. Liang, X. Shen and X. Lin, "GRS: The Green, Reliability, and Security of Emerging Machine to Machine Communications," IEEE Communications Magazine, vol. 49, no. 4, pp. 28-35, 2011.

[36] P. Kasinathan, C. Pastrone, M. A. Spirito and M. Vinkovits, "Denial-of-Service detection in 6LoWPAN based Internet of Things," Proceedings of 2013 IEEE 9th International Conference on Wireless and Mobile Computing, Networking and Communications (WiMob), pp. 600-607, 2013.

[37] K. Krentz and G. Wunder, "6LoWPAN Security: Avoiding Hidden Wormholes using Channel Reciprocity," Proceedings of Proceedings of the 4th International Workshop on Trustworthy Embedded Devices, USA, pp. 13-22, 2014.

[38] G. Lai, "Detection of Wormhole Attacks on IPv6 Mobility-based Wireless Sensor Network," EURASIP Journal on Wireless Communications and Networking, vol. 2016, no. 1-11, p. 274, 2016.

[39] C. Cervantes, D. Poplade, M. Nogueira and A. Santos, "Detection of Sinkhole Attacks for Supporting Secure Routing on 6LoWPAN for Internet of Things," Proceedings of 2015 IFIP/IEEE International Symposium on Integrated 
Network Management (IM), pp. 606-611, 2015.

[40] S. Raza, L. Wallgren and T. Voigt, "SVELTE: Real-time intrusion detection in the Internet of Things," Ad Hoc Networks, vol. 11, no. 8, pp. 2661-2674, 2013.

[41] X. Wang and Y. Mu, "A Secure Mobility Support Scheme for 6LoWPAN Wireless Sensor Networks," Security and Communication Networks, vol. 7, no. 3, pp. 641-652, 2014.

[42] H. R. Hussen, G. A. Tizazu, M. Ting, T. Lee, Y. Choi and K. Kim, "SAKES: Secure Authentication and Key Establishment Scheme for M2M Communication in the IP-based Wireless Sensor nNetwork (6LoWPAN)," Proceedings of 2013 Fifth International Conference on Ubiquitous and Future Networks (ICUFN), pp. 246-251, 2013.

[43] L. M. L. Oliveira, J. J. P. C. Rodrigues, A. F. d. Sousa and V. M. Denisov, "Network Admission Control Solution for 6LoWPAN Networks Based on Symmetric Key Mechanisms," IEEE Transactions on Industrial Informatics, vol. 12, no. 6, pp. 2186-2195, 2016.

[44] R. Hummen, J. Hiller, H. Wirtz, M. Henze, H. Shafagh and K. Wehrle, "6LoWPAN Fragmentation Attacks and Mitigation Mechanisms," Proceedings of Proceedings of the sixth ACM conference on Security and privacy in wireless and mobile networks, Hungary, pp. 55-66, 2013.

[45] H. Zhou, H. Zhang and Y. Qi, "An Authentication Method for Proxy Mobile IPv6 and Performance Analysis," Security and Communication Networks, vol. 2, no. 5, pp. 445-454, 2009.

[46] M. C. Chuang, J. F. Lee and M. C. Chen, "SPAM: A Secure Password Authentication Mechanism for Seamless Handover in Proxy Mobile IPv6 Networks," IEEE Systems Journal, vol. 7, no. 1, pp. 102-113, 2013.

[47] I. You and F. Y. Leu, "Comments on "SPAM: A Secure Password Authentication Mechanism for Seamless Handover in Proxy Mobile IPv6 Networks"," IEEE Systems Journal, vol. PP, no. 99, pp. 1-4, 2015.

[48] M. C. Chuang and J. F. Lee, "SF-PMIPv6: A Secure Fast Handover Mechanism for Proxy Mobile IPv6 networks," Journal of Systems and Software, vol. 86, no. 2, pp. 437-448, 2013.

[49] K. F. Krentz, H. Rafiee and C. Meinel, "6LoWPAN Security: Adding Compromise Resilience to the 802.15.4 Security Sublayer," Proceedings of Proceedings of the International Workshop on Adaptive Security, Switzerland, pp. 1-10, 2013.

[50] X. Wang and Y. Mu, "Addressing and Privacy Support for 6LoWPAN," IEEE Sensors Journal, vol. 15, no. 9, pp. 5193-5201, 2015.

[51] X. Wang, H. Cheng and Y. Yao, "Addressing With an Improved DAD for 6LoWPAN," IEEE Communications Letters, vol. 20, no. 1, pp. 73-76, 2016. 
[52] S. Kent and K. Seo, "Security Architecture for the Internet Protocol," IETF RFC 4301, 2005.

[53] J. Granjal, E. Monteiro and J. S. Silva, "Enabling Network-Layer Security on IPv6 Wireless Sensor Networks," Proceedings of 2010 IEEE Global Telecommunications Conference (GLOBECOM), pp. 1-6, 2010.

[54] S. Kent and R. Atkinson, "IP Authentication Header," IETF RFC 2402, 1998.

[55] S. Kent and R. Atkinson, "IP Encapsulating Security Payload (ESP)," IETF RFC 2406, 1998.

[56] S. Raza, S. Duquennoy, T. Chung, D. Yazar, T. Voigt and U. Roedig, "Securing Communication in 6LoWPAN with Compressed IPsec," Proceedings of 2011 International Conference on Distributed Computing in Sensor Systems and Workshops (DCOSS), pp. 1-8, 2011.

[57] S. Raza, S. Duquennoy, J. Höglund, U. Roedig and T. Voigt, "Secure Communication for the Internet of Things-A Comparison of Link-layer Security and IPsec for 6LoWPAN," Security and Communication Networks, vol. 7, no. 12, pp. 2654-2668, 2014.

[58] A. Rghioui, M. Bouhorma and A. Benslimane, "Analytical Study of Security Aspects in 6LoWPAN Networks," Proceedings of 2013 5th International Conference on Information and Communication Technology for the Muslim World (ICT4M), pp. 1-5, 2013.

[59] J. Granjal, E. Monteiro and J. S. Silva, "Security Issues and Approaches on Wireless M2M Systems," Wireless Networks and Security: Issues, Challenges and Research Trends, S. Khan and A. K. Pathan, Eds., Berlin Heidelberg: Springer Berlin Heidelberg, pp. 133-164, 2013.

[60] J. W. Hui and D. E. Culler, "Extending IP to Low-Power, Wireless Personal Area Networks," IEEE Internet Computing, vol. 12, no. 4, pp. 37-45, 2008.

[61] X. Sun, S. Men, C. Zhao and Z. Zhou, "A Security Authentication Scheme in Machine-to-Machine Home Network Service," Security and Communication Networks, vol. 8, no. 16, pp. 2678-2686, 2015.

[62] C. Shuo and M. Maode, "A Dynamic-Encryption Authentication Scheme for M2M Security in Cyber-Physical Systems," Proceedings of 2013 IEEE Global Communications Conference (GLOBECOM), pp. 2897-2901, 2013.

[63] A. Fu, Y. Zhang, Z. Zhu, Q. Jing and J. Feng, "An Efficient Handover Authentication Scheme with Privacy Preservation for IEEE 802.16m Network," Computers \& Security, vol. 31, no. 6, pp. 741-749, 2012.

[64] X. Zhang, G. Li and W. Han, "Ticket-Based Authentication for Fast Handover in Wireless Mesh Networks," Wireless Personal Communications, vol. 85, no. 3, pp. 1509-1523, 2015.

[65] D. Dolev and A. C. Yao, "On the Security of Public Key Protocols," 
Proceedings of 22nd Annual Symposium on Foundations of Computer Science (SFCS 1981), pp. 350-357, 1981.

[66] Z. Shelby, S. Chakrabarti, E. Nordmark and C. Bormann, "Neighbor Discovery Optimization for IPv6 over Low-Power Wireless Personal Area Networks (6LoWPANs)," IETF RFC 6775, 2012.

[67] A. Datta, A. Derek, J. C. Mitchell and A. Roy, "Protocol Composition Logic (PCL)," Electronic Notes in Theoretical Computer Science, vol. 172, pp. 311358, 2007.

[68] C. Cremers, "On the Protocol Composition Pogic (PCL)," Proceedings of Proceedings of the 2008 ACM Symposium on Information, Computer and Communications Security, Japan, pp. 66-76, 2008.

[69] The AVISPA Team, AVISPA v1.1 User Manual, 2006. [Online]. Available: http://www.avispa-project.org/package/user-manual.pdf.

[70] M. Turuani, "The CL-Atse Protocol Analyser," Term Rewriting and Applications, F. Pfenning, Ed., Berlin, Heidelberg: Springer Berlin Heidelberg, pp. 277-286, 2006.

[71] D. Basin, S. Mödersheim and L. Viganò, "OFMC: A Symbolic Model Checker for Security Protocols," International Journal of Information Security, vol. 4, no. 3, pp. 181-208, 2005.

[72] W. Backes and J. Cordasco, "MoteAODV - An AODV Implementation for TinyOS 2.0," Information Security Theory and Practices. Security and Privacy of Pervasive Systems and Smart Devices. vol. 6033, P. Samarati, M. Tunstall, J. Posegga, K. Markantonakis, and D. Sauveron, Eds., Berlin Heidelberg: Springer Berlin Heidelberg, pp. 154-169, 2010.

[73] K. Piotrowski, P. Langendoerfer and S. Peter, "How Public Key Cryptography Influences Wireless Sensor Node Lifetime," Proceedings of Proceedings of the Fourth ACM Workshop on Security of Ad Hoc and Sensor Networks, USA, pp. 169-176, 2006.

[74] H. Yokota, K. Chowdhury, R. Koodli, B. Patil and F. Xia, "Fast Handovers for Proxy Mobile IPv6," IETF RFC 5949, 2010.

[75] S. Park, J. E. Lee, J. Choi and Y. Kim, "Fast Localized Proxy Mobile IPv6 (FLPMIPv6)," IETF draft-park-netlmm-fastpmip-00, 2007.

[76] A. K. Quoc, D. S. Kim and H. Choo, "A Novel Scheme for Preventing Out-OfOrder Packets in Fast Handover for Proxy Mobile IPv6," Proceedings of The International Conference on Information Networking 2014 (ICOIN 2014), pp. 422-427, 2014.

[77] A. Ahmad and D. Sasidharan, "Handover Efficiency Improvement in Proxy Mobile IPv6 (PMIPv6) Networks," Procedia Computer Science, vol. 46, pp. 1064-1071, 2015. 
[78] L. Y. Yeh, J. G. Chang, W. Huang and Y. L. Tsai, "A Localized Authentication and Billing Scheme for Proxy Mobile IPv6 in VANETs," Proceedings of 2012 IEEE International Conference on Communications (ICC), pp. 993-998, 2012.

[79] S. Céspedes, S. Taha and X. Shen, "A Multihop-Authenticated Proxy Mobile IP Scheme for Asymmetric VANETs," IEEE Transactions on Vehicular Technology, vol. 62, no. 7, pp. 3271-3286, 2013.

[80] L. Y. Yeh and Y. C. Lin, "A Proxy-Based Authentication and Billing Scheme With Incentive-Aware Multihop Forwarding for Vehicular Networks," IEEE Transactions on Intelligent Transportation Systems, vol. 15, no. 4, pp. 16071621, 2014.

[81] K. Grover and A. Lim, "A Survey of Broadcast Authentication Schemes for Wireless Networks," Ad Hoc Networks, vol. 24, Part A, pp. 288-316, 2015.

[82] Y. S. Chen, C. S. Hsu and H. K. Lee, "An Enhanced Group Mobility Protocol for 6LoWPAN-Based Wireless Body Area Networks," IEEE Sensors Journal, vol. 14, no. 3, pp. 797-807, 2014.

[83] A. Fu, S. Lan, B. Huang, Z. Zhu and Y. Zhang, "A Novel Group-Based Handover Authentication Scheme with Privacy Preservation for Mobile WiMAX Networks," IEEE Communications Letters, vol. 16, no. 11, pp. 17441747, 2012.

[84] J. Cao, H. Li and M. Ma, "GAHAP: A Group-based Anonymity Handover Authentication Protocol for MTC in LTE-A Networks," Proceedings of 2015 IEEE International Conference on Communications (ICC), pp. 3020-3025, 2015.

[85] J. Li, M. Wen and T. Zhang, "Group-Based Authentication and Key Agreement With Dynamic Policy Updating for MTC in LTE-A Networks," IEEE Internet of Things Journal, vol. 3, no. 3, pp. 408-417, 2016.

[86] J. Cao, H. Li, M. Ma and F. Li, "UGHA: Uniform Group-based Handover Authentication for MTC within E-UTRAN in LTE-A Networks," Proceedings of 2015 IEEE International Conference on Communications (ICC), pp. 72467251, 2015.

[87] F. Gont, "A Method for Generating Semantically Opaque Interface Identifiers with IPv6 Stateless Address Autoconfiguration (SLAAC)," IETF RFC 7217, 2014.

[88] M. Burrows, M. Abadi and R. Needham, "A logic of authentication," $A C M$ Trans. Comput. Syst., vol. 8, no. 1, pp. 18-36, 1990.

[89] C. Cremers, Scyther User Manual, 2014. [Online]. Available: http://profs.info.uaic.ro/ cbirjoveanu/web/Ps/Scyther/scyther-manual.pdf.

[90] G. d. Meulenaer, F. Gosset, F. X. Standaert and O. Pereira, "On the Energy Cost of Communication and Cryptography in Wireless Sensor Networks," 
Proceedings of 2008 IEEE International Conference on Wireless and Mobile Computing, Networking and Communications, pp. 580-585, 2008.

[91] C. Gomez, J. Paradells and J. Crowcroft, "Optimized 6LoWPAN Fragmentation Header for LPWAN," IETF draft-gomez-lpwan-fragmentation-header-00, 2016.

[92] J. Kim, R. Haw, E. J. Cho, C. S. Hong and S. Lee, "A 6LoWPAN Sensor Node Mobility Scheme Based on Proxy Mobile IPv6," IEEE Transactions on Mobile Computing, vol. 11, no. 12, pp. 2060-2072, 2012.

[93] W. A. Simpson, T. Narten, E. Nordmark and H. Soliman, "Neighbor Discovery for IP version 6 (IPv6)," IETF RFC 4861, 2007. 


\section{List of Abbreviation}

\begin{tabular}{|c|c|}
\hline 6LER & 6LoWPAN Edge Router \\
\hline $6 \mathrm{LH}$ & 6LoWPAN Host \\
\hline 6LoWPAN & IPv6 over Low-Power Wireless Personal Area Networks \\
\hline $6 \mathrm{LR}$ & 6LoWPAN Router \\
\hline $6 \mathrm{MAG}$ & 6LoWPAN MAG \\
\hline $6 \mathrm{MN}$ & 6LoWPAN MN \\
\hline AAA & Authentication, Authorization and Accounting \\
\hline $\mathrm{ACK}$ & Acknowledgement \\
\hline AES & Advanced Encryption Standard \\
\hline $\mathrm{AH}$ & Authentication Header \\
\hline AKE & Authentication and Key Establishment \\
\hline AP & Access Point \\
\hline APKES & Adaptable Pairwise Key Establishment Scheme \\
\hline AVISPA & Automated Validation of Internet Security Protocols and Applications \\
\hline BAN & Burrows-Abadi-Needham \\
\hline BPBA & Bicasting PBA \\
\hline BPBU & Bicasting PBU \\
\hline $\mathrm{BS}$ & Base Station \\
\hline $\mathrm{CBC}$ & Cipher Block Chaining \\
\hline CB-PMIPv6 & Chaining based PMIPv6 \\
\hline CL-Atse & Constraint Logic Based Attack Searcher \\
\hline $\mathrm{CN}$ & Core Network \\
\hline CPS & Cyber-Physical System \\
\hline CPU & Central Processing Unit \\
\hline CTR & Counter \\
\hline DAD & Duplicate Address Detection \\
\hline
\end{tabular}




$\begin{array}{ll}\text { De-PBA } & \text { De-registration PBA } \\ \text { De-PBU } & \text { De-registration PBU } \\ \text { DMN } & \text { Dishonest MN } \\ \text { DoS } & \text { Denial-of-Service } \\ \text { E2E } & \text { End-to-End } \\ \text { EAKES6Lo } & \text { Enhanced Authentication and Key Establishment Scheme in } \\ & \text { 6LoWPAN } \\ \text { EAP } & \text { Extensible Authentication Protocol } \\ \text { ECC } & \text { Elliptic Curve Cryptography } \\ \text { ECDH } & \text { Elliptic Curve Diffie-Hellman } \\ \text { ECDSA } & \text { Elliptic Curve Digital Signature Algorithm } \\ \text { EDGE } & \text { Enhanced Data Rates for GSM Evolution } \\ \text { ESP } & \text { Encapsulated Security Payload } \\ \text { ESPH } & \text { Efficient and Secure PMIPv6-based Handover } \\ \text { ETSI } & \text { The European Telecommunications Standards Institute } \\ \text { EUI } & \text { Extended Unique Identifier } \\ \text { FA } & \text { Foreign Agent } \\ \text { FFD } & \text { Full-Function Device } \\ \text { FLPMIPv6 } & \text { Fast and Localized PMIPv6 } \\ \text { FPMIPv6 } & \text { Fast Handovers for PMIPv6 } \\ \text { GB } & \text { Gigabyte } \\ \text { GERAN } & \text { GSM EDGE Radio Access Network } \\ \text { GHz } & \text { Gigahertz } \\ \text { GSM } & \text { Global System for Mobile Communications } \\ \text { HA } & \text { Home Agent } \\ \text { HACK } & \text { Handover Acknowledgement } \\ \text { HI } & \text { Handover Initiate } \\ \text { HLPSL } & \text { High Level Protocol Specification Language } \\ \text { HMAC } & \text { Hash-based Message Authentication Code } \\ \text { HNP } & \text { Home Network Prefix } \\ \text { HA }\end{array}$




\begin{tabular}{|c|c|}
\hline IDS & Intrusion Detection System \\
\hline IEEE & Institute of Electrical and Electronics Engineers \\
\hline IETF & Internet Engineering Task Force \\
\hline IF & Intermediate Format \\
\hline IID & Interface Identifier \\
\hline IKE & Internet Key Exchange \\
\hline IKEv2 & Internet Key Exchange Protocol version 2 \\
\hline INTI & Intrusion Detection of Sinkhole Attacks on 6LoWPAN for IoT \\
\hline IoT & Internet of Things \\
\hline IP & Internet Protocol \\
\hline IPHC & IPv6 Header Compression \\
\hline IPsec & Internet Protocol Security \\
\hline IPv6 & Internet Protocol Version 6 \\
\hline LMA & Local Mobility Anchor \\
\hline LoWPAN & Low-Power Wireless Personal Area Network \\
\hline LTE & Long Term Evolution \\
\hline LTE-A & LTE-Advanced \\
\hline M2M & Machine-to-Machine \\
\hline $\mathrm{MAC}^{1}$ & Media Access Control \\
\hline $\mathrm{MAC}^{2}$ & Message Authentication Code \\
\hline MAG & Mobile Access Gateway \\
\hline MATLAB & Matrix Laboratory \\
\hline M-BUS & Meter-Bus \\
\hline MIHF & Media Independent Handover Function \\
\hline MIPv6 & Mobile IPv6 \\
\hline MITM & Man-in-the-Middle \\
\hline $\mathrm{MN}$ & Mobile Node \\
\hline MR & Mobile Router \\
\hline MS & Mobile Station \\
\hline N6MAG & New 6LoWPAN MAG \\
\hline
\end{tabular}




\begin{tabular}{ll} 
NEMO & Network Mobility \\
NETLMM & Network-based Localized Mobility Management \\
NMAG & New MAG \\
OFMC & On-The-Fly Model Checker \\
OPNET & Optimized Network Engineering Tools \\
P6MAG & Previous 6LoWPAN MAG \\
PAN & Personal Area Network \\
PBA & Proxy Binding Acknowledgement \\
PBU & Proxy Binding Update \\
PCL & Protocol Composition Logic \\
PKI & Public Key Infrastructure \\
PLC & Power Line Communications \\
PMAG & Previous MAG \\
PMIPv6 & Proxy Mobile IPv6 \\
QoS & Quality of Service \\
RA & Router Advertisement \\
RAM & Random Access Memory \\
RFD & Reduced-Function Device \\
RPL & Routing Protocol for Low-Power and Lossy Networks \\
RS & Router Solicitation \\
RSSI & Received Signal Strength Indicator \\
RX & Receive \\
SAKES & Secure Authentication and Key Establishment Scheme \\
SCREWED & Secure Channel Reciprocity-based Wormhole Detection \\
SCs & Service Capabilities \\
SGMS & Secure Group Mobility Scheme \\
SHA & Secure Hash Algorithm \\
SPAM & Secure Password Authentication Mechanism \\
SPDL & Security Protocol Language Description \\
SVELTE & Elegantly Slim \\
\hline
\end{tabular}




$\begin{array}{ll}\text { SYN } & \text { Synchronize } \\ \text { TC } & \text { Technical Committee } \\ \text { TCP } & \text { Transmission Control Protocol } \\ \text { TID } & \text { Temporary Identifier } \\ \text { TX } & \text { Transmit } \\ \text { UDP } & \text { User Datagram Protocol } \\ \text { WBAN } & \text { Wireless Body Area Network } \\ \text { WiMAX } & \text { Worldwide Interoperability for Microwave Access } \\ \text { W-LAN } & \text { Wireless Local Area Network } \\ \text { xDSL } & \text { Digital Subscriber Line Technologies } \\ \text { XOR } & \text { Exclusive or }\end{array}$

